\title{
Calorimetry for particle physics
}

\author{
Christian W. Fabjan and Fabiola Gianotti \\ CERN, 1211 Geneva 23, Switzerland
}

(Published 15 October 2003)

\begin{abstract}
Calorimetry has become a well-understood, powerful, and versatile measurement method. Besides perfecting this technique to match increasingly demanding operation at high-energy particle accelerators, physicists are developing low-temperature calorimeters to extend detection down to ever lower energies, and atmospheric and deep-sea calorimeters to scrutinize the universe up to the highest energies. The authors summarize the state of the art, with emphasis on the physics of the detectors and innovative technologies.
\end{abstract}

\section{CONTENTS}

I. Introduction

II. Electromagnetic Calorimetry

A. Physics of the electromagnetic cascade

B. Energy resolution of electromagnetic calorimeters

1. Stochastic term

2. Noise term

3. Constant term

4. Additional contributions

C. Main techniques and examples of facilities

1. Homogeneous calorimeters

a. Semiconductor calorimeters

b. Cherenkov calorimeters

c. Scintillation calorimeters

d. Noble-liquid calorimeters

2. Sampling calorimeters

a. Scintillation sampling calorimeters

b. Gas sampling calorimeters

c. Solid-state sampling calorimeters

d. Liquid sampling calorimeters

III. Hadron Calorimetry
A. Physics of the hadronic cascade
B. Energy resolution of hadron calorimeters
C. Monte Carlo codes for hadronic cascade simulation
1. Shower physics modeling techniques
2. Applications: Illustrative examples
D. Examples of hadron calorimeter facilities

IV. Calorimeter Operation in Accelerator Experiments

A. Performance requirements

B. Integration

1. Impact of material

2. Particle identification

C. Calorimeter calibration

V. Low-Temperature Calorimeters

A. Introduction

B. Main technologies

1. Thermal detectors

2. Phonon sensors

3. Superheated superconducting granules

C. Representative applications

1. Search for dark matter

2. Neutrinoless double-beta decay

3. Microcalorimeters for $\mathrm{x}$-ray astronomy

4. Superconducting tunneling junctions for ultraviolet to infrared spectroscopy in astronomy
VI. Citius, Altius, Fortius

A. Introduction 1280

B. Atmospheric calorimeters 1280

1. Setting the energy scale 1283

2. Energy resolution 1283

C. Deep-water calorimeters 1283

VII. Conclusions 1284

Acknowledgments 1284

References 1284

\section{INTRODUCTION}

Calorimetry is an ubiquitous detection principle in particle physics. Originally invented for the study of cosmic-ray phenomena, this method was developed and perfected for accelerator-based particle physics experimentation primarily in order to measure the energy of electrons, photons, and hadrons. Calorimeters are blocks of instrumented material in which particles to be measured are fully absorbed and their energy transformed into a measurable quantity. The interaction of the incident particle with the detector (through electromagnetic or strong processes) produces a shower of secondary particles with progressively degraded energy. The energy deposited by the charged particles of the shower in the active part of the calorimeter, which can be detected in the form of charge or light, serves as a measurement of the energy of the incident particle.

Calorimeters can be broadly divided into electromagnetic calorimeters, used mainly to measure electrons and photons through their electromagnetic interactions (e.g., bremsstrahlung, pair production), and hadronic calorimeters, used to measure mainly hadrons through their strong and electromagnetic interactions. They can be further classified according to their construction technique into sampling calorimeters and homogeneous calorimeters. Sampling calorimeters consist of alternating layers of an absorber, a dense material used to degrade the energy of the incident particle, and an active medium that provides the detectable signal. Homogeneous calorimeters, on the other hand, are built of only one type of material that performs both tasks, energy degradation and signal generation.

Today particle physics reaches ever higher energies of experimentation, and aims to record complete event in- 
formation. Calorimeters are attractive in this field for various reasons:

- In contrast with magnetic spectrometers, in which the momentum resolution deteriorates linearly with the particle momentum, in most cases the calorimeter energy resolution improves with energy as $1 / \sqrt{E}$, where $E$ is the energy of the incident particle. Therefore calorimeters are very well suited to high-energy physics experiments.

- In contrast with magnetic spectrometers, calorimeters are sensitive to all types of particles, charged and neutral (e.g., neutrons). They can even provide indirect detection of neutrinos and their energy through a measurement of the event missing energy.

- They are versatile detectors. Although originally conceived as devices for energy measurement, they can be used to determine the shower position and direction, to identify different particles (for instance, to distinguish electrons and photons from pions and muons on the basis of their different interactions with the detector), and to measure the arrival time of the particle. Calorimeters are also commonly used for trigger purposes, since they can provide fast signals that are easy to process and to interpret.

- They are space and therefore cost effective. Because the shower length increases only logarithmically with energy, the detector thickness needs to increase only logarithmically with the energy of the particles. In contrast, for a fixed momentum resolution, the bending power $B L^{2}$ of a magnetic spectrometer (where $B$ is the magnetic field and $L$ the length) must increase linearly with the particle momentum $p$.

Besides perfecting this technique to match the physics potential at the major particle accelerator facilities, remarkable extensions have been made to explore new energy domains. Low-temperature calorimeters, sensitive to phonon excitations, detect particles with unprecedented energy resolution and are sensitive to very-lowenergy deposits which cannot be detected in conventional devices. The quest to understand the origin, composition, and spectra of energetic cosmic rays has led to imaginative applications in which the atmosphere or the sea are instrumented over thousands of cubic kilometers.

A regular series of conferences (CALOR, 2002) and a comprehensive recent monograph (Wigmans, 2000) testify to the vitality of this field.

In this paper we review major calorimeter developments with emphasis on applications at high-energy accelerators. First, the physics, the performance, and practical realizations of electromagnetic calorimetry are discussed (Sec. II). Next, the physics of hadronic calorimeters and the processes determining their performance are presented (Sec. III). A section on calorimetry for accelerators (Sec. IV) concludes with a discussion of integration issues. Section $\mathrm{V}$ is dedicated to an overview of low-temperature calorimeters. The achievements and projects in atmospheric and water calorimeters are analyzed in Sec. VI. Section VII is devoted to the conclusions.

The success of calorimeters in modern experiments rests also on remarkable developments in the field of high-performance readout electronics that have allowed optimum exploitation of the intrinsic potential of these detectors. A discussion of calorimeter readout techniques is beyond the scope of this paper. A very good review has been made by de La Taille (2000).

\section{ELECTROMAGNETIC CALORIMETRY}

In this section we discuss the physics and the performance of electromagnetic calorimeters. The main techniques used to build these detectors are also reviewed, and their merits and drawbacks are described. Examples of calorimeters operated at recent or present highenergy physics experiments, or under construction for future machines, are given as illustration.

\section{A. Physics of the electromagnetic cascade}

In spite of the apparently complex phenomenology of shower development in a material, electrons and photons interact with matter via a few well-understood QED processes, and the main shower features can be parametrized with simple empirical functions.

The average energy lost by electrons in lead and the photon interaction cross section are shown in Fig. 1 as a function of energy. Two main regimes can be identified. For energies larger than $\sim 10 \mathrm{MeV}$, the main source of electron energy loss is bremsstrahlung. In this energy range, photon interactions produce mainly electronpositron pairs. For energies above $1 \mathrm{GeV}$ both these processes become roughly energy independent. At low energies, on the other hand, electrons lose their energy mainly through collisions with the atoms and molecules of the material thus giving rise to ionization and thermal excitation; photons lose their energy through Compton scattering and the photoelectric effect.

As a consequence, electrons and photons of sufficiently high energy ( $\geqslant 1 \mathrm{GeV}$ ) incident on a block of material produce secondary photons by bremsstrahlung, or secondary electrons and positrons by pair production. These secondary particles in turn produce other particles by the same mechanisms, thus giving rise to a cascade (shower) of particles with progressively degraded energies. The number of particles in the shower increases until the energy of the electron component falls below a critical energy $\epsilon$, where energy is dissipated mainly by ionization and excitation and not in the generation of other particles.

The main features of electromagnetic showers (e.g., their longitudinal and lateral sizes) can be described in terms of one parameter, the radiation length $X_{0}$, which depends on the characteristics of the material (Particle Data Group, 2002), 

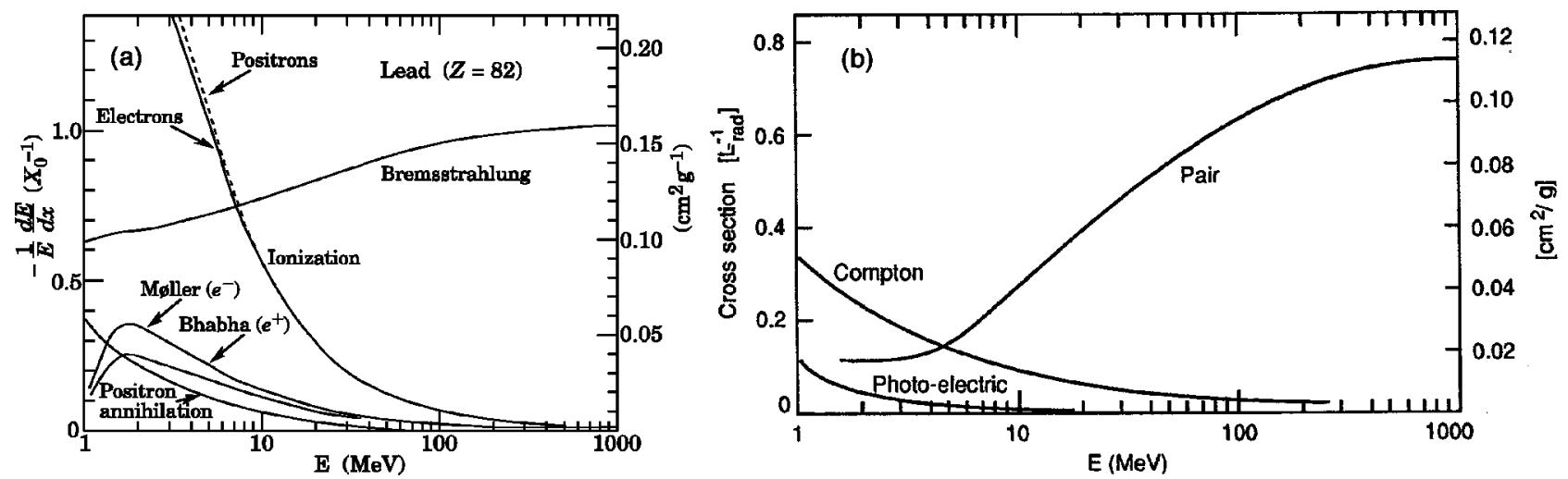

FIG. 1. (a) Fractional energy lost in lead by electrons and positrons as a function of energy (Particle Data Group, 2002). (b) Photon interaction cross section in lead as a function of energy (Fabjan, 1987).

$$
X_{0}\left(\mathrm{~g} / \mathrm{cm}^{2}\right) \simeq \frac{716 \mathrm{~g} \mathrm{~cm}^{-2} A}{Z(Z+1) \ln (287 / \sqrt{Z})},
$$

where $Z$ and $A$ are the atomic number and weight of the material, respectively. The radiation length governs the rate at which electrons lose energy by bremsstrahlung, since it represents the average distance $x$ that an electron needs to travel in a material to reduce its energy to $1 / e$ of its original energy $E_{0}$,

$$
\langle E(x)\rangle=E_{0} e^{-x / X_{0}}
$$

Similarly, a photon beam of initial intensity $I_{0}$ traversing a block of material is absorbed mainly through pair production. After traveling a distance $x=\frac{9}{7} X_{0}$, its intensity is reduced to $1 / e$ of the original intensity,

$$
\langle I(x)\rangle=I_{0} e^{-(7 / 9)\left(x / X_{0}\right)} .
$$

Two slightly different definitions are used for the critical energy $\epsilon$. In the first one, $\epsilon$ is the energy at which the electron ionization losses and bremsstrahlung losses become equal. This energy depends on the features of the material and is approximately given by

$$
\epsilon=\frac{610(710) \mathrm{MeV}}{Z+1.24(0.92)}
$$

for solids (gases). Figure 1 shows that $\epsilon \sim 7 \mathrm{MeV}$ in lead. In the second definition (Rossi, 1952), $\epsilon$ is the energy at which the ionization loss per $X_{0}$ equals the electron energy $E$ :

$$
\frac{d E}{d x}(\text { ionization })=\frac{E}{X_{0}} .
$$

Both definitions are equivalent in the approximation

$$
\frac{d E}{d x}(\text { bremsstrahlung }) \simeq \frac{E}{X_{0}} \text {. }
$$

Equations (2) and (3) show that the physical scale over which a shower develops is similar for incident electrons and photons, and is independent of the material type if expressed in terms of $X_{0}$. Therefore electromagnetic showers can be described in a universal way by using simple functions of the radiation length.
For instance, the mean longitudinal profile can be described (Longo and Sestili, 1975)

$$
\frac{d E}{d t}=E_{0} b \frac{(b t)^{a-1} e^{-b t}}{\Gamma(a)},
$$

where $t=x / X_{0}$ is the depth inside the material in radiation lengths and $a$ and $b$ are parameters related to the nature of the incident particle $\left(e^{ \pm}\right.$or $\left.\gamma\right)$. The shower maximum, i.e., the depth at which the largest number of secondary particles is produced, is approximately located at

$$
t_{\max } \simeq \ln \frac{E_{0}}{\epsilon}+t_{0}
$$

where $t_{\max }$ is measured in radiation lengths, $E_{0}$ is the incident particle energy, and $t_{0}=-0.5(+0.5)$ for electrons (photons). This formula shows the logarithmic dependence of the shower length, and therefore of the detector thickness needed to absorb a shower, on the incident particle energy. Longitudinal shower profiles for different energies of the incident particles are shown in Fig. 2 (left plot). The calorimeter thickness containing $95 \%$ of the shower energy is approximately given by

$$
t_{95 \%} \simeq t_{\max }+0.08 Z+9.6,
$$

where $t_{\max }$ and $t_{95 \%}$ are measured in radiation lengths. In calorimeters with thickness $\simeq 25 X_{0}$, the shower longitudinal leakage beyond the end of the active detector is much less than $1 \%$ up to incident electron energies of $\sim 300 \mathrm{GeV}$. Therefore, even at the particle energies expected at the CERN Large Hadron Collider (LHC), of order $\sim \mathrm{TeV}$, electromagnetic calorimeters are very compact devices: the ATLAS lead-liquid argon calorimeter (ATLAS Collaboration, 1996b) and the CMS crystal calorimeter (CMS Collaboration, 1997) have thicknesses of $\simeq 45 \mathrm{~cm}$ and $\simeq 23 \mathrm{~cm}$, respectively (the radiation lengths are $\simeq 1.8 \mathrm{~cm}$ and $\simeq 0.9 \mathrm{~cm}$, respectively).

The transverse size of an electromagnetic shower is mainly due to multiple scattering of electrons and positrons away from the shower axis. Bremsstrahlung photons emitted by these electrons and positrons can also 

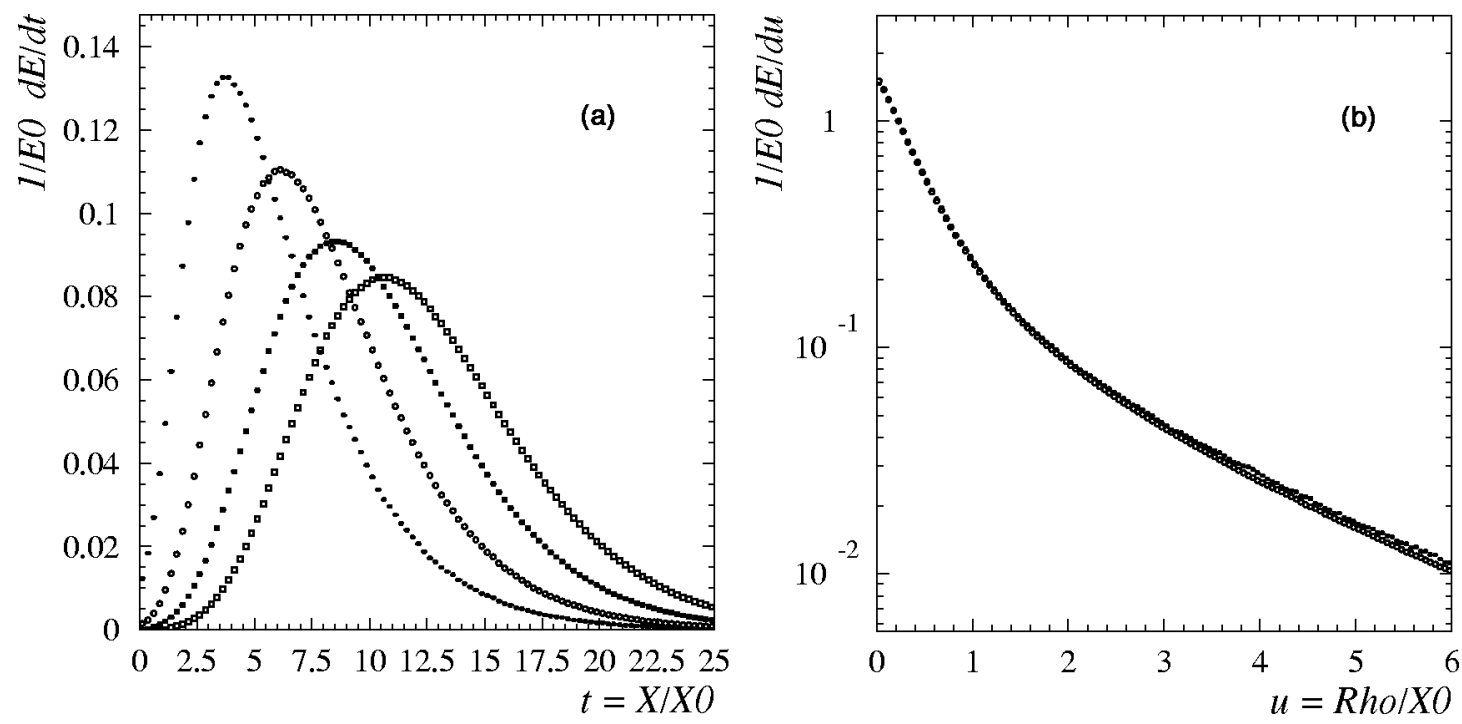

FIG. 2. (a) Simulated shower longitudinal profiles in $\mathrm{PbWO}_{4}$, as a function of the material thickness (expressed in radiation lengths), for incident electrons of energy (from left to right) $1 \mathrm{GeV}, 10 \mathrm{GeV}, 100 \mathrm{GeV}, 1 \mathrm{TeV}$. (b) Simulated radial shower profiles in $\mathrm{PbWO}_{4}$, as a function of the radial distance from the shower axis (expressed in radiation lengths), for $1 \mathrm{GeV}$ (closed circles) and $1 \mathrm{TeV}$ (open circles) incident electrons. From Maire (2001).

contribute to the shower spread. A measurement of the transverse size, integrated over the full shower depth, is given by the Molière radius $\left(R_{M}\right)$, which can be approximated by

$$
R_{M}\left(\mathrm{~g} / \mathrm{cm}^{2}\right) \simeq 21 \mathrm{MeV} \frac{X_{0}}{\epsilon(\mathrm{MeV})} .
$$

It represents the average lateral deflection of electrons at the critical energy after traversing one radiation length. The definition of critical energy as given in Eq. (5) should be used here, since it more accurately describes the transverse electromagnetic shower development (Particle Data Group, 2002). On average, about $90 \%$ of the shower energy is contained in a cylinder of radius $\sim 1 R_{M}$. Since for most calorimeters $R_{M}$ is of the order of a few centimeters, electromagnetic showers are quite narrow. In addition, their transverse size is roughly energy independent. An example of shower radial profile is presented in Fig. 2 (right plot). The cells of a segmented calorimeter must be comparable in size to (or smaller than) one $R_{M}$ if the calorimeter is to be used for precision measurements of the shower position.

\section{B. Energy resolution of electromagnetic calorimeters}

The measurement of energy with an electromagnetic calorimeter is based on the principle that the energy released in the detector material by the charged particles of the shower, mainly through ionization and excitation, is proportional to the energy of the incident particle.

The total track length of the shower $T_{0}$, defined as the sum of all ionization tracks due to all charged particles in the cascade, is proportional to

$$
T_{0}\left(\mathrm{~g} / \mathrm{cm}^{2}\right) \propto X_{0} \frac{E_{0}}{\epsilon},
$$

where the symbol $\propto$ indicates proportionality and $E_{0} / \epsilon$ is the number of particles in the shower. The above formula shows that a measurement of the signal produced by the charged tracks of the cascade provides a measurement of the original particle energy $E_{0}$. This measurement can be performed, for instance, by detecting the light produced in a scintillating material, or by collecting the charge produced in a gas or in a liquid.

The intrinsic energy resolution of an ideal calorimeter, that is, a calorimeter with infinite size and no response deterioration due to instrumental effects (for example, inefficiencies in the signal collection, mechanical nonuniformities), is mainly due to fluctuations of the track length $T_{0}$. Since $T_{0}$ is proportional to the number of track segments in the shower, and the shower development is a stochastic process, the intrinsic energy resolution is given, from purely statistical arguments, by

$$
\sigma(E) \propto \sqrt{T_{0}},
$$

from which the well-known dependence of the fractional energy resolution on energy,

$$
\frac{\sigma(E)}{E} \propto \frac{1}{\sqrt{T_{0}}} \propto \frac{1}{\sqrt{E_{0}}},
$$

can be derived.

The actual energy resolution of a realistic calorimeter is deteriorated by other contributions and can be written in a more general way as

$$
\frac{\sigma}{E}=\frac{a}{\sqrt{E}} \oplus \frac{b}{E} \oplus c,
$$

where the symbol $\oplus$ indicates a quadratic sum. The first term on the right-hand side is called the stochastic term, and includes the shower intrinsic fluctuations mentioned above; the second term is the noise term; and the 
third term is the constant term. The relative importance of the various terms depends on the energy of the incident particle. Therefore the optimal calorimeter technique can be very different for experiments operating in different energy ranges, since the energy resolution is dominated by different contributions. These contributions are discussed in turn below.

\section{Stochastic term}

As already mentioned, this term is due to the fluctuations related to the physical development of the shower.

In homogeneous calorimeters intrinsic fluctuations are small because the energy deposited in the active volume of the detector by an incident monochromatic beam of particles does not fluctuate event by event. Therefore in most cases the intrinsic energy resolution can be better than the statistical expectation given in Eq. (12) by a factor called the Fano factor (Fano, 1947). The experimental evidence for Fano factors in semiconductor, noble gas, and noble-liquid calorimeters for charge or light collection is discussed in several papers (Alkhazov et al., 1967; Doke et al., 1976; Seguinot et al., 1995). Typical stochastic terms of homogeneous electromagnetic calorimeters are at the level of a few percent in units of $1 / \sqrt{E(\mathrm{GeV})}$ and are dominated by effects other than the intrinsic resolution (Secs. II.B.3 and II.B.4).

On the other hand, in sampling calorimeters the energy deposited in the active medium fluctuates event by event because the active layers are interleaved with absorber layers. These fluctuations, which are called sampling fluctuations and represent the most important limitation to the energy resolution of these detectors, are due to variations in the number of charged particles $N_{\mathrm{ch}}$ that cross the active layers. This number is proportional to

$$
N_{\mathrm{ch}} \propto \frac{E_{0}}{t}
$$

where $t$ is the thickness of the absorber layers in radiation lengths. If one assumes statistically independent crossings of the active layers, which is reasonable if the absorber layers are not too thin, then the "sampling" contribution to the energy resolution comes from the fluctuation of $N_{\mathrm{ch}}$, that is (Amaldi, 1981)

$$
\frac{\sigma}{E} \propto \frac{1}{\sqrt{N_{\mathrm{ch}}}} \propto \sqrt{\frac{t}{E_{0}(\mathrm{GeV})}} .
$$

The smaller the thickness $t$, the larger the number of times the shower is sampled by the active layers (i.e., the sampling frequency) and the number of detected particles, the better the energy resolution. Hence in principle the energy resolution of a sampling calorimeter can be improved by reducing the thickness of the absorber layers. However, in order to achieve resolutions comparable to those typical of homogeneous calorimeters, absorber thicknesses of a few percent of a radiation length are needed, but this is rarely feasible in practice. Although some approximations have been used to derive Eq. (16), this simplified approach is nevertheless able to demonstrate the energy dependence of the resolution. More complete discussions can be found, for instance, in Wigmans (2000).

The typical energy resolution of sampling electromagnetic calorimeters is in the range $5-20 \% / \sqrt{E(\mathrm{GeV})}$.

Another parameter of sampling calorimeters is the sampling fraction $f_{\text {samp }}$, which has an impact on the noise term of the energy resolution (Sec. II.B.2):

$$
f_{\text {samp }}=\frac{E_{\text {mip }}(\text { active })}{E_{\text {mip }}(\text { active })+E_{\text {mip }}(\text { absorber })},
$$

where $E_{\text {mip }}\left(\right.$ active) and $E_{\text {mip }}$ (absorber) indicate the energies deposited by an incident minimum-ionizing particle in the active part and in the absorber part of the detector, respectively.

\section{Noise term}

This contribution to the energy resolution comes from the electronic noise of the readout chain and depends on the detector technique and on the features of the readout circuit (detector capacitance, cables, etc.).

Calorimeters in which the signal is collected in the form of light, such as scintillator-based sampling or homogeneous calorimeters, can achieve small levels of noise if the first step of the electronic chain is a photosensitive device, like a phototube, which provides a high-gain multiplication of the original signal with almost no noise.

On the other hand, the noise is larger in detectors in which the signal is collected in the form of charge because the first element of the readout chain is a preamplifier. Techniques like signal shaping and optimal filtering are used to minimize the electronic noise in these detectors (Cleland and Stern, 1994). Nevertheless, a fundamental limitation remains. This can be schematically described by the relation $Q=\sqrt{4 k T R \delta F}$ (where $Q$ is the equivalent noise charge, $k$ the Boltzmann constant, $T$ the temperature, $R$ the equivalent noise resistance of the preamplifier, and $\delta F$ the bandwidth), which shows that the noise increases when one wants to operate at a high rate.

The noise contribution to the energy resolution increases with decreasing energy of the incident particles [see Eq. (14)] and at energies below a few GeV may become dominant. Therefore the noise equivalent energy is usually required to be much smaller than 100 $\mathrm{MeV}$ per channel for applications in the several $\mathrm{GeV}$ region.

In sampling calorimeters the noise term can be decreased by increasing the sampling fraction, because the larger the sampling fraction, the larger the signal from the active medium and therefore the higher the signalto-noise ratio.

\section{Constant term}

This term includes contributions that do not depend on the energy of the particle. Instrumental effects that cause variations of the calorimeter response with the particle impact point on the detector give rise to re- 


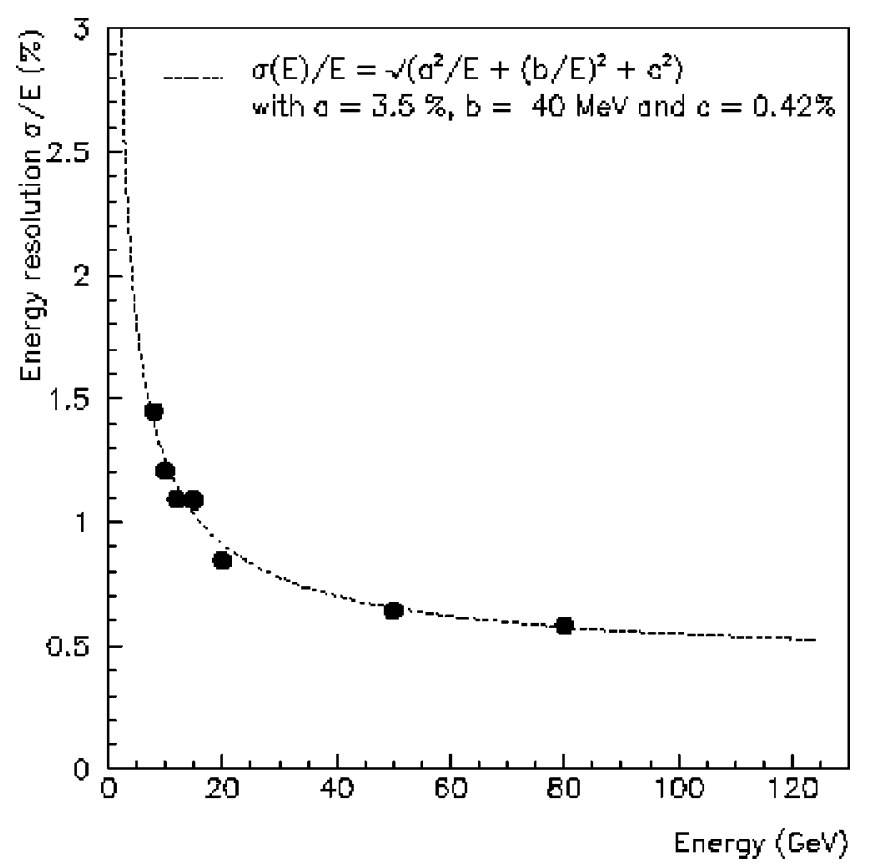

FIG. 3. Fractional electron energy resolution as a function of energy measured with a prototype of the NA48 liquid krypton electromagnetic calorimeter (NA48 Collaboration, 1995). The line is a fit to the experimental points with the form and the parameters indicated in the figure.

sponse nonuniformities. These latter contribute an additional smearing to the measured energy of particles distributed over large calorimeter areas, which results in a constant term. Nonuniformities can originate from the detector geometry (for instance, if the absorber and active layers have irregular shapes), from imperfections in the detector mechanical structure and readout system, from temperature gradients, from the detector aging, from radiation damage, etc. These nonuniformities can be cured (to a large extent) if they exhibit a periodic pattern, as is the case if they are related to the detector geometry, or if they originate from the readout chain (this is the task of the calibration procedure discussed in Sec. IV.C). On the other hand, other effects such as mechanical imperfections are randomly distributed and therefore more difficult to correct.

With the increasing energy of present and future accelerators, the constant term becomes more and more the dominant contribution to the energy resolution of electromagnetic calorimeters. Tight construction tolerances are therefore imposed on the mechanics and readout system of modern calorimeters, for instance, LHC calorimeters.

Typically the constant term of an electromagnetic calorimeter should be kept at the level of $1 \%$ or smaller. This is particularly true for homogeneous calorimeters, because of their small stochastic term.

Figure 3 shows the energy resolution measured with a prototype of the NA48 liquid krypton electromagnetic calorimeter (see Sec. II.C.1). The experimental points are fitted with the form given in Eq. (14).

\section{Additional contributions}

Additional contributions to the energy resolution come from the constraints to which a calorimeter is subjected when integrated in a big experiment (Sec. IV.B). Examples are the following.

- Longitudinal leakage. Space and cost constraints limit the thickness of a calorimeter operating in a highenergy physics experiment. Therefore energetic showers can lose part of their energy beyond the end of the active calorimeter volume. This leakage fluctuates event by event, thus deteriorating the energy resolution. This effect can be in part corrected by weighting the energy deposited by the showers in the last compartment of a longitudinally segmented calorimeter.

- Lateral leakage. In order to limit the contribution of the electronic noise, of the event pileup when operating at high-luminosity machines (see Sec. IV.A), and of other particles in the same physics event, a relatively small cluster of calorimeter cells is usually used to reconstruct an electromagnetic shower. As a consequence, a fraction of the shower energy can be lost outside this cluster. This fraction fluctuates event by event, and therefore introduces an additional smearing in the energy measurement. The choice of the optimum cluster size is obviously the result of a trade-off among the above-mentioned effects.

- Upstream energy losses. Calorimeters are supported by mechanical structures and equipped with cables and electronics. In addition, when operating inside an experiment, they are usually preceded by other detectors, such as tracking devices. As a consequence, electrons and photons coming from the interaction region have to traverse a non-negligible amount of inactive material before reaching the active volume of the calorimeter. The energy lost in this material fluctuates event by event, and these fluctuations deteriorate the energy resolution. Possible techniques to recover part of these losses (e.g., the use of dedicated devices like presamplers and massless gaps) are discussed in Sec. IV.B.1.

- Nonhermetic coverage. Cracks and dead regions are often present inside the calorimeter volume because big detectors are usually built of mechanically independent modules and divided into barrel and forward parts. The quality of the energy measurement is degraded for showers developing in these inactive areas, resulting in a deterioration of the energy resolution and in the appearance of low-energy tails in the reconstructed energy spectra of incident particles. This can also have an impact on the quality of the missing transverse energy measurement (see Sec. IV.A).

The above effects often dominate the resolution of homogeneous calorimeters operated in high-energy physics experiments, because of their excellent stochastic term. 


\section{Main techniques and examples of facilities}

In this section we discuss the main techniques used to build homogeneous and sampling electromagnetic calorimeters, and the advantages and drawbacks of the various solutions. Examples of detectors in operation or under construction are given.

\section{Homogeneous calorimeters}

The main advantage of these detectors is their excellent energy resolution, which is due to the fact that the whole energy of an incident particle is deposited in the active medium, in contrast with sampling calorimeters. On the other hand, homogeneous calorimeters can be less easily segmented laterally and longitudinally, which is a drawback when position measurements and particle identification are needed. Furthermore, since these devices are noncompensating (see Sec. III.B), and suitable materials have a large interaction length (see Sec. III.B), thereby making the detector thickness needed to contain hadron showers prohibitive, homogeneous calorimeters are rarely used as hadronic calorimeters in accelerator experiments. On the other hand, they are employed in neutrino and astroparticle physics experiments in which large volumes are needed to detect rare events, and therefore only homogeneous detectors made of inexpensive materials (like water or air) are affordable.

Homogeneous calorimeters can be broadly divided into four classes:

- Semiconductor calorimeters. In this case, the ionization tracks produce electron-hole pairs in the material valence and conduction bands that give rise to an electric signal. These detectors provide an excellent energy resolution. Examples are silicon and germanium crystals used in many nuclear physics applications.

- Cherenkov calorimeters. The medium is a transparent material in which relativistic $e^{ \pm}$in the shower produce Cherenkov photons. The signal is therefore collected in the form of light. Lead-glass calorimeters are a widely used example.

- Scintillator calorimeters. The medium is a material in which ionization tracks produce light (fluorescence). Examples are $\mathrm{BGO}$, CsI, and $\mathrm{PbWO}_{4}$ crystals.

- Noble-liquid calorimeters. The medium is a noble gas (Ar, Kr, Xe) operated at cryogenic temperature. Although in this case both ionization and scintillation signals can in principle be collected, large-scale calorimeters for high-energy physics applications are based on the charge measurement.

In detectors where the signal is collected in the form of light (Cherenkov, scintillators), photons from the active volume are converted into electrons (usually called photoelectrons) by a photosensitive device such as a photomultiplier. A contribution to the energy resolution can come from statistical fluctuations in the number of photoelectrons. This contribution has the form $\propto 1 / \sqrt{N_{\text {pe }}}$, where $N_{\text {pe }}$ is the number of photoelectrons, and is important if $N_{\mathrm{pe}}$ is small. The number of photoelectrons can be small if the number of photons produced in the active medium is small, as is the case in Cherenkov calorimeters, or if there are losses in the light collection. Furthermore, the efficiency of the device converting photons into electrons (the photocathode in the case of a photomultiplier) is usually in the range $20-70 \%$. In addition, if the amplification of the electric signal is relatively small, the contribution of the electronic noise to the energy resolution becomes important. This is not a problem if photomultipliers that have gains of order $10^{6}$ are used, but could be a problem with photodiodes, which have gains in the range 1-10. Unfortunately, when operating in a high magnetic field, standard photomultipliers cannot be used since their gain and linearity are affected by the field. In conclusion, maximization of the light yield is an important issue in the design, construction, and operation of homogeneous calorimeters.

Another crucial aspect is the minimization and accurate control of all possible sources of response nonuniformities, which otherwise could give rise to a large constant term of the energy resolution, thereby spoiling the excellent intrinsic resolution of these detectors.

\section{a. Semiconductor calorimeters}

These calorimeters are rarely used in high-energy physics experiments for various reasons. They are expensive and therefore not suited to large systems. They have an excellent intrinsic resolution that is optimal for low-energy particles, whereas at high energy other effects like leakage and response nonuniformities dominate. They are, however, extensively used as photon detectors for nuclear physics applications, in particular, gamma spectroscopy. They are briefly mentioned here because of their excellent energy resolution, better than the stochastic limit discussed in Sec. II.B.

The energy $W$ needed to create an electron-hole pair is about $3.6 \mathrm{eV}$ in $\mathrm{Si}$ and $2.9 \mathrm{eV}$ in $\mathrm{Ge}$ (at a temperature of $77 \mathrm{~K}$ ). Since the incident particle energy is fully absorbed and most of it is converted into such pairs, an incident monochromatic photon beam of fixed energy $E_{0}$ produces an almost fixed number of electron-hole pairs given approximately by $N_{\text {eh }} \simeq E_{0} / W$. Therefore the signal provided by the detector fluctuates very little from event to event and the intrinsic energy resolution can be parametrized as

$$
\frac{\sigma}{E} \propto \frac{\sqrt{F}}{\sqrt{N_{\mathrm{eh}}}},
$$

where $F$, the Fano factor, is smaller than unity (e.g., $F$ $\simeq 0.13$ in Ge). For instance, photons of energy $1 \mathrm{MeV}$ incident on a Ge crystal produce a number of electronhole pairs $N_{\mathrm{eh}} \sim 3.3 \times 10^{5}$. An energy resolution scaling like $1 / \sqrt{N_{\text {eh }}}$ [see Eq. (13)] would give $\sigma(E) \simeq 1.7 \mathrm{keV}$, whereas one obtains $\sigma(E) \simeq 630 \mathrm{eV}$ by including the Fano factor. The measured value $\sigma(E) \simeq 550 \mathrm{eV}$ (Knoll, 1989 ) is in good agreement with this latter prediction. The much superior energy resolution of semiconductor detectors compared to other calorimeters is illustrated in 


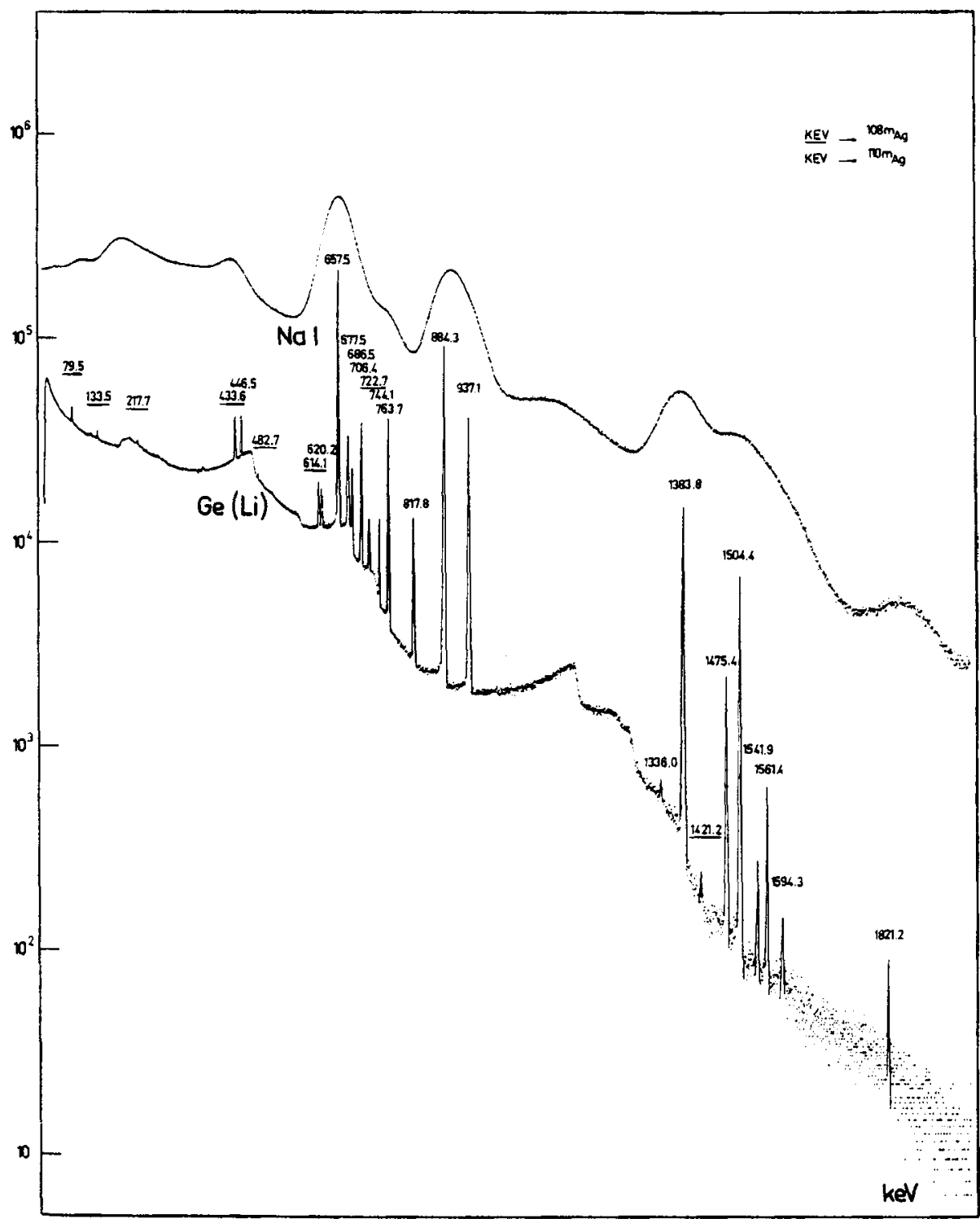

FIG. 4. Spectral lines of a $\mathrm{Ag} \gamma$ source as measured with a $\mathrm{Ge}$ crystal and a $\mathrm{NaI}(\mathrm{Tl})$ scintillator. The peaks are labeled in keV. Adapted from Knoll (1989).
Fig. 4, which shows the spectral lines of a Ag $\gamma$ source as measured with a $\mathrm{NaI}(\mathrm{Tl})$ scintillator (one of the scintillators with the best energy resolution) and with a Ge crystal.

\section{b. Cherenkov calorimeters}

Detectable Cherenkov light is produced whenever a particle traverses a transparent medium with a speed $v$ $>c / n$, where $c / n$ is the speed of light in that medium and $n$ is the refractive index of the medium. Cherenkov light is emitted on the surface of a cone centered on the particle trajectory and with half angle $\theta_{C}=\arccos (c / n v)$. Dielectric materials with $n>1$ are good candidates for Cherenkov detectors.

These devices are usually employed for particle identification purposes, since the emission of Cherenkov light depends on the particle's velocity, and therefore on its mass for a given momentum $p$. However, they can also be used as calorimeters by collecting the light produced by relativistic $e^{ \pm}$tracks in the showers.
Lead glass $(\mathrm{PbO})$ is cheap and easy to handle and therefore has been widely used in the past for highenergy physics applications, for example, in the NOMAD (Altegoer et al., 1998) neutrino experiment at the CERN SPS and in the OPAL (Akrawy et al., 1990) experiment at LEP. One drawback of $\mathrm{PbO}$ is the poor radiation resistance, since a significant deterioration of the light output is observed for doses larger than $\sim 100$ Gy. Therefore other materials are being considered today, such as lead fluoride $\left(\mathrm{PbF}_{2}\right)$, which overcomes this problem and also offers other advantages like a smaller radiation length and a higher light output.

Cherenkov calorimeters usually have a worse energy resolution than other types of homogeneous calorimeters. This is mainly due to the fact that the light yield is small (usually $10^{4}$ times smaller than in a scintillator), because only shower tracks with $v>c / n$ produce a detectable signal. In addition, the maximum photon intensity is obtained for short wavelengths (typically $\lambda$ $<300-350 \mathrm{~nm}$ ), whereas most photocathodes are sensitive to the 300-600-nm region. As an example, about 
1000 photoelectrons are produced in lead glass per deposited $\mathrm{GeV}$, which alone (i.e., without taking into account possible inefficiencies in the signal collection and other effects like shower containment) gives an energy resolution of $\sim 3 \% / \sqrt{E(\mathrm{GeV})}$. Optimization efforts have therefore been made to maximize the light yield of large-scale detectors operating at colliders. The OPAL end-cap lead glass calorimeter has achieved a light yield of $\sim 1800$ photoelectrons/GeV and an energy resolution (taking into account all effects) of order $5 \% / \sqrt{E(\mathrm{GeV})}$.

As an example of nonaccelerator application, the Super-Kamiokande water Cherenkov detector consists of $50 \mathrm{kton}$ of pure water viewed by about 12000 photomultipliers (Super-Kamiokande Collaboration, 1999). Its size is optimized for the study of neutrino interactions. One of the goals of the experiment was to measure the flux of ${ }^{8} \mathrm{~B}$ solar neutrinos by detecting electrons in the energy range 5-20 MeV. A remarkable energy resolution of about $20 \%$ has been achieved for $10-\mathrm{MeV}$ electrons from neutrino interactions. At this energy the signal yield is $\sim 60$ photoelectrons. Since it is very important to avoid distortions in the measured electron spectra, the detector response stability with time and response uniformity across the active volume must be controlled to $\pm 0.5 \%$. This has been achieved by using electrons of several energies injected at different places in the detector. These are provided by a precisely calibrated LINAC installed on the top of the tank.

\section{c. Scintillation calorimeters}

Scintillators can be divided into two classes, organic and inorganic, characterized by two different physical mechanisms for light emission and by different advantages and drawbacks. Organic scintillators are fast but suffer from a poor light yield; inorganic scintillators offer a large light yield and good signal linearity, but usually have a slow response.

Devices based on organic scintillators are usually binary or ternary systems consisting of an organic solvent (e.g., a mineral oil) with a small fraction (typically $\leqslant 1 \%$ ) of a scintillating solute (fluors). The molecules of the solvent are excited by an incident charged particle and transfer the excitation to the solute (for instance, through dipole interactions), which produces the detectable signal. Without fluors, the base material would reabsorb a large part of the emitted light. This would give rise to an unacceptably short light attenuation length (the attenuation length is the distance that the emitted light has to travel in the scintillator before its intensity is reduced to $1 / e$ of the original intensity). To further increase the light yield and collection efficiency, a "wavelength-shifting" material can also be added as a third component. The process of excitation, molecular transfer, and light emission is very fast, of the order of a few nanoseconds. However, the light output is relatively small because the solute concentration is small. The use of organic scintillators for homogeneous calorimeters is very limited, mainly because they are not dense enough, whereas they are commonly chosen as the active medium for sampling calorimeters (see Sec. II.C.2).

In inorganic scintillators the light emission is related to the crystal structure of the material. Incident charged particles produce electron-hole pairs in the conduction and valence bands of the medium, and photons are emitted when electrons return to the valence band. The frequency of the emitted radiation and the response time depend on the gap between the valence and the conduction bands and on the details of the electron migration in the lattice structure. They vary a lot from material to material. Often, in order to increase the light yield (for example, by matching the signal wavelength to the photocathode spectral sensitivity) and to obtain a faster response, crystals are doped with tiny amounts of impurities. These dopants, the most commonly used of which is thallium (Tl), create additional activation sites in the gap between the valence band and the conduction band. These sites can be filled by electrons from the conduction band, and can therefore increase the emission probability and change the light wavelength and the material decay time. The intrinsic energy resolution is better than that of Cherenkov calorimeters because the light yield is several orders of magnitude larger thanks to the lower energy needed to create an electron-hole pair compared to that needed to exceed the Cherenkov threshold, and to the higher efficiency of the photon emission mechanism. Nevertheless, minimization of inefficiencies in the light collection, which could arise from reflections, photon absorption, or bad matching between optical elements, is a crucial issue in some scintillator calorimeters with small light yield (e.g., $\mathrm{PbWO}_{4}$ ).

One drawback of crystal detectors is that they are not intrinsically uniform. Indeed, it is not easy to grow the thousands of ingots needed for a big calorimeter system in an identical way, nor to ensure the same light collection efficiency in all of them. This could give rise to response variations from crystal to crystal which, if not minimized and controlled with adequate calibration systems, could translate into a large constant term in the energy resolution.

Table I summarizes the main properties of the crystals most commonly used for high-energy physics applications. $\mathrm{NaI}(\mathrm{Tl})$ has been widely employed in the past because of its low cost and large light yield. However, it is hygroscopic and has a relatively long radiation length, not well suited to big experiments where denser materials like $\mathrm{BGO}$ and $\mathrm{PbWO}_{4}$, which allow more compact detectors, are preferred. CsI is also very popular, being used, for example, by the BaBar (Boutigny et al., 1995), Belle (Abashian et al., 2002), CLEO (Bebek et al., 1988), and $\mathrm{KTeV}$ (Alavi-Harati et al., 1999) experiments. It has a short radiation length, is easier to handle than NaI, and if doped with thallium it offers the second largest light yield of all crystals after NaI. Pure CsI has a fast component (6 ns), which is well adapted to high-rate experiments, and therefore was used for instance by $\mathrm{KTeV}$. When doped with thallium it becomes much slower, but the light yield increases significantly. For this reason $\mathrm{CsI}(\mathrm{Tl})$ has been chosen by the BaBar experi- 
TABLE I. Main properties of crystals commonly used for homogeneous electromagnetic calorimeters in accelerator experiments.

\begin{tabular}{lccccc}
\hline \hline & $\mathrm{NaI}(\mathrm{Tl})$ & $\mathrm{CsI}(\mathrm{Tl})$ & $\mathrm{CsI}$ & $\mathrm{BGO}$ & $\mathrm{PbWO}_{4}$ \\
\hline Density $\left(\mathrm{g} / \mathrm{cm}^{3}\right)$ & 3.67 & 4.53 & 4.53 & 7.13 & 8.28 \\
$X_{0}(\mathrm{~cm})$ & 2.59 & 1.85 & 1.85 & 1.12 & 0.89 \\
$R_{M}(\mathrm{~cm})$ & 4.5 & 3.8 & 3.8 & 2.4 & 2.2 \\
Decay time (ns) & 250 & 1000 & 10 & 300 & 5 \\
$\quad$ slow component & & & 36 & & 15 \\
Emission peak (nm) & 410 & 565 & 305 & 410 & 440 \\
$\quad$ slow component & & & 480 & & \\
Light yield $\gamma / \mathrm{MeV}$ & $4 \times 10^{4}$ & $5 \times 10^{4}$ & $4 \times 10^{4}$ & $8 \times 10^{3}$ & $1.5 \times 10^{2}$ \\
Photoelectron yield & 1 & 0.4 & 0.1 & 0.15 & 0.01 \\
$\quad($ relative to NaI) & & & & & $10^{5}$ \\
Rad. hardness $(\mathrm{Gy})$ & 1 & 10 & $10^{3}$ & 1 & \\
\hline \hline
\end{tabular}

ment (see below), which does not need a fast calorimeter but a calorimeter with a large light yield, sensitive to low-energy signals. Finally $\mathrm{PbWO}_{4}$ (lead tungstate), which is very dense, fast, and radiation hard, is the crystal best suited to the LHC environment and has been adopted by the CMS experiment (CMS Collaboration, 1997).

We discuss below in more detail examples of homogeneous scintillator calorimeters used in modern highenergy physics experiments.

The choice of the calorimeter technique for the BaBar experiment (Boutigny et al., 1995) at the SLAC PEP-II B-factory was dictated by the goal of reconstructing lowenergy (down to $\sim 10 \mathrm{MeV}$ ) photons and $\pi^{0}$ 's from $B$-meson decays with high efficiency, in order to be sensitive to rare decays. Excellent energy and position (and therefore mass) resolutions are also needed in order to achieve a good signal-to-background ratio for these decays. A CsI(Tl) calorimeter offers very good energy resolution and large light output. The signal yield of the BaBar calorimeter is $\sim 7000$ photoelectrons $/ \mathrm{MeV}$, which allows small noise levels $(\simeq 230 \mathrm{keV}$ per crystal $)$ and high detection efficiency at very low energies $(\simeq 95 \%$ for $20-\mathrm{MeV}$ photons). The long decay time of $\mathrm{CsI}(\mathrm{Tl})$, $\sim \mu \mathrm{s}$, is not a limitation given the relatively low interaction rate $(\simeq 100 \mathrm{~Hz})$ at the SLAC B-factory. The calo- rimeter consists of 6580 crystals covering the polar angle $-0.78<\cos \theta<0.96$ (PEP-II is an asymmetric machine), as schematically shown in Fig. 5. The (tapered) crystals have a constant thickness of $\simeq 17 X_{0}$ for particles coming from the interaction region, a trapezoidal face of transverse size $5 \times 5 \mathrm{~cm}^{2}$, and no longitudinal segmentation. They do not point to the interaction center, so that photon losses in gaps between crystals are minimized. For reasons of redundancy and efficiency each channel is read out by two Si photodiodes (the calorimeter is inside a magnetic field of $1.5 \mathrm{~T}$ ), followed by preamplifiers, shapers, and ADC's (analog-to-digital converters). A cell-to-cell response dispersion of only $\simeq 0.25 \%$ should be achieved by using an electronic calibration system, a Xe pulser, a radioactive source, and physics events at the collider (Bhabha, $e^{+} e^{-} \rightarrow \gamma \gamma$, etc.). A preliminary energy resolution of $\simeq 2 \% / E^{1 / 4} \oplus 1.8 \%$ has been achieved (BaBar Collaboration, 2000), with contributions from photoelectron statistics, longitudinal leakage, lateral shower fluctuations outside the area of $5 \times 5$ crystals used to reconstruct a shower, energy losses in the upstream dead material (amounting to $<0.5 X_{0}$ ), light collection nonuniformities, cell-to-cell calibration spread, electronic and beam background noise. All these effects contribute to the $\sim 1 / E^{1 / 4}$ energy dependence of the resolution. Figure 6 shows the reconstructed two-photon

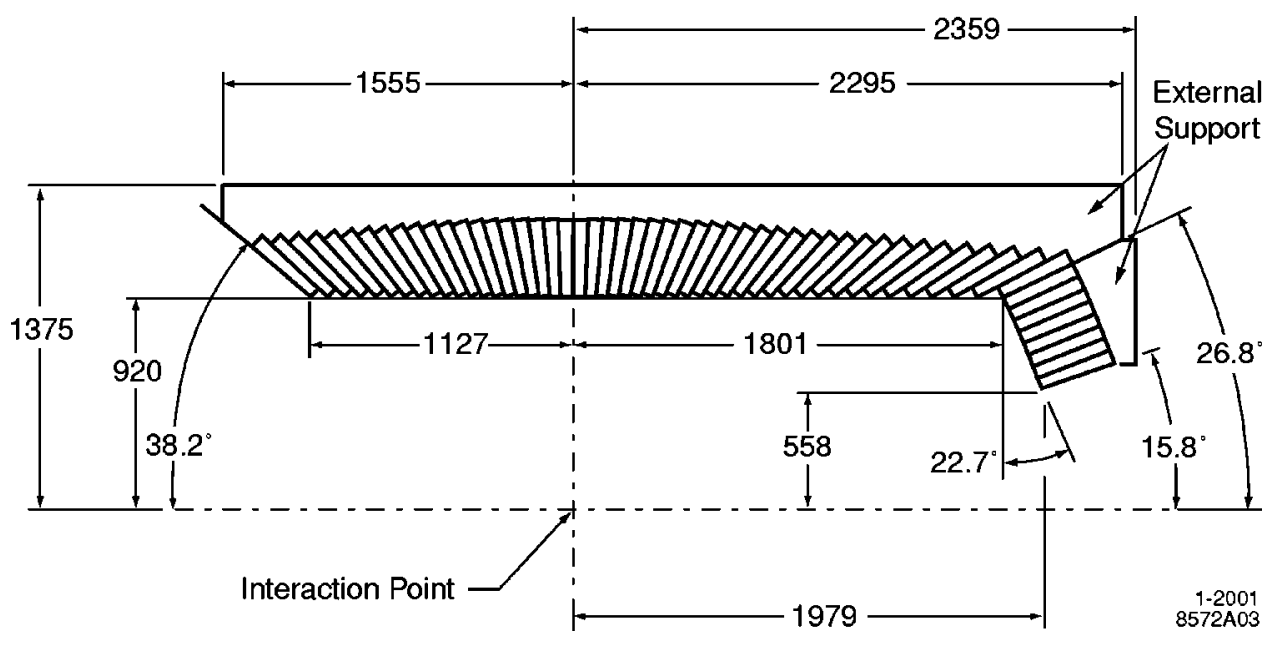

FIG. 5. View of the BaBar electromagnetic crystal calorimeter. All dimensions are in millimeters. 
$\times 10^{2}$
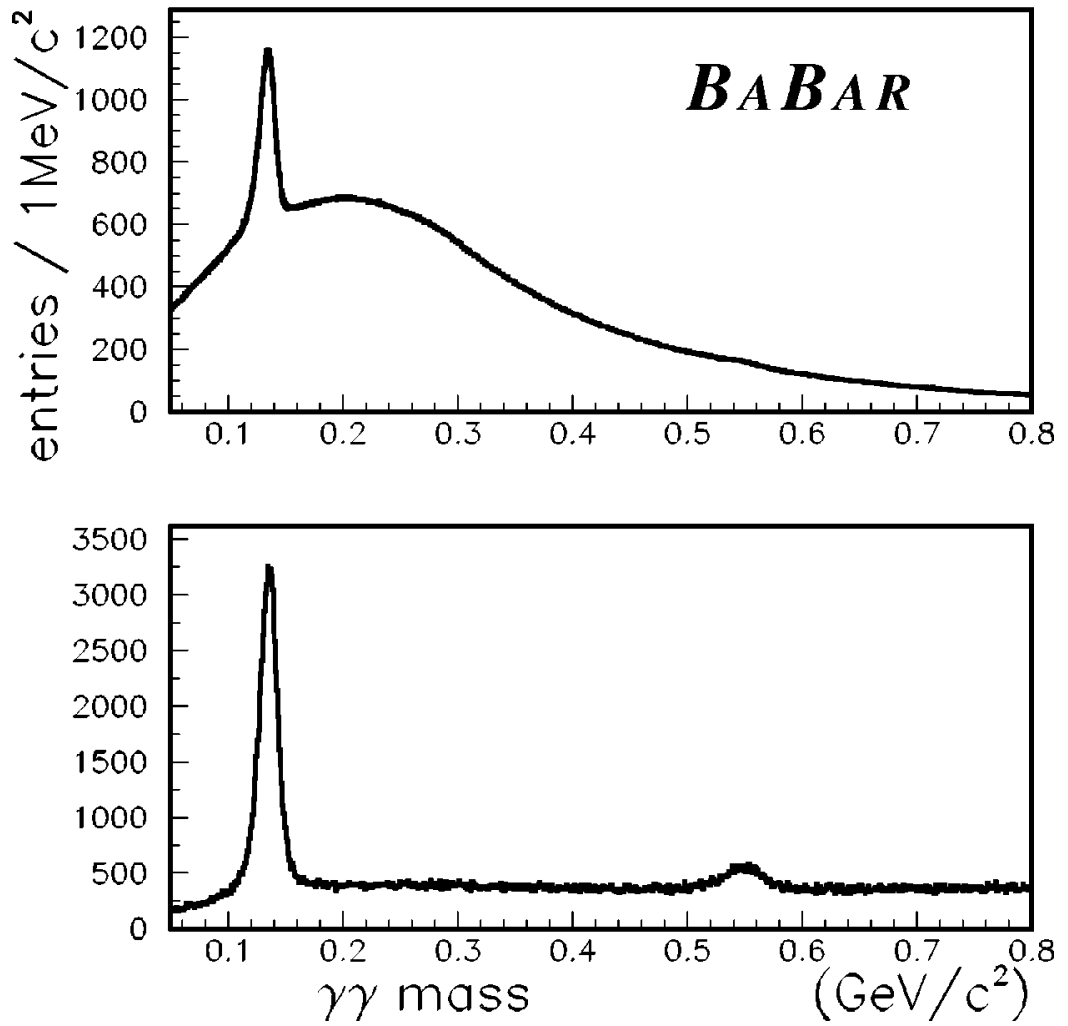

FIG. 6. The invariant mass of $\gamma \gamma$ pairs from hadronic $B$-meson events in BaBar with $E_{\gamma}$ $>30 \mathrm{MeV}$ and $E_{\gamma \gamma}>300 \mathrm{MeV}$ (top plot), and $E_{\gamma}>100 \mathrm{MeV}$ and $E_{\gamma \gamma}>1 \mathrm{GeV}$ (bottom plot). Peaks due to $\pi^{0}$ and $\eta$ production are visible. From BaBar Collaboration (2000). invariant mass obtained for $B$-meson events. The $\pi^{0}$ mass resolution measured in the data $(6.9 \mathrm{MeV})$ is in excellent agreement with the Monte Carlo expectation (6.8 MeV).

Bismuth germanate $\left(\mathrm{Bi}_{4} \mathrm{Ge}_{3} \mathrm{O}_{12}\right.$, or $\left.\mathrm{BGO}\right)$ has been used for the electromagnetic calorimeter of the L3 experiment at LEP (Bakken et al., 1985). This calorimeter, which consists of about 10000 crystals of transverse size $2 \times 2 \mathrm{~cm}^{2}$, is the crystal detector with the largest number of channels operated so far. The energy resolution obtained with test beam data is $1.5 \% / \sqrt{E(\mathrm{GeV})} \oplus 0.4 \%$, whereas the resolution measured at LEP over the full calorimeter acceptance with Bhabha electrons ( $E$ $\simeq 45 \mathrm{GeV}$ ) is $1.2 \%$, thus indicating a constant term of order $1 \%$ (see Fig. 7). This larger than expected constant term has been attributed to temperature effects, cell-to-cell calibration spread, electrons impinging near the crystal boundaries, etc. It demonstrates that the control of the response uniformity in crystal calorimeters is a crucial and difficult issue, especially in big systems.

The CMS electromagnetic calorimeter (CMS Collaboration, 1997) consists of about $83000 \mathrm{PbWO}_{4}$ crystals covering the rapidity region $|\eta| \leqslant 3$. The crystals have a transverse size of $\simeq 2 \times 2 \mathrm{~cm}^{2}$ and no longitudinal segmentation. This technique has been chosen because of the excellent energy resolution, which is important, for instance, in the search for a possible $H \rightarrow \gamma \gamma$ signal (see Sec. IV.A). As shown in Table I, lead tungstate exhibits some features which make it particularly suited to the LHC environment. It has a very short radiation length, which allows an active thickness of $\simeq 26 X_{0}$ to be fitted in a radial space as short as $23 \mathrm{~cm}$; a small Molière ra- dius, which ensures small lateral shower size; high radiation resistance; and fast response since $\sim 80 \%$ of the light is emitted in less than $15 \mathrm{~ns}$. The main drawback is that the light yield is quite modest, which requires a very careful optimization of the signal collection system. The

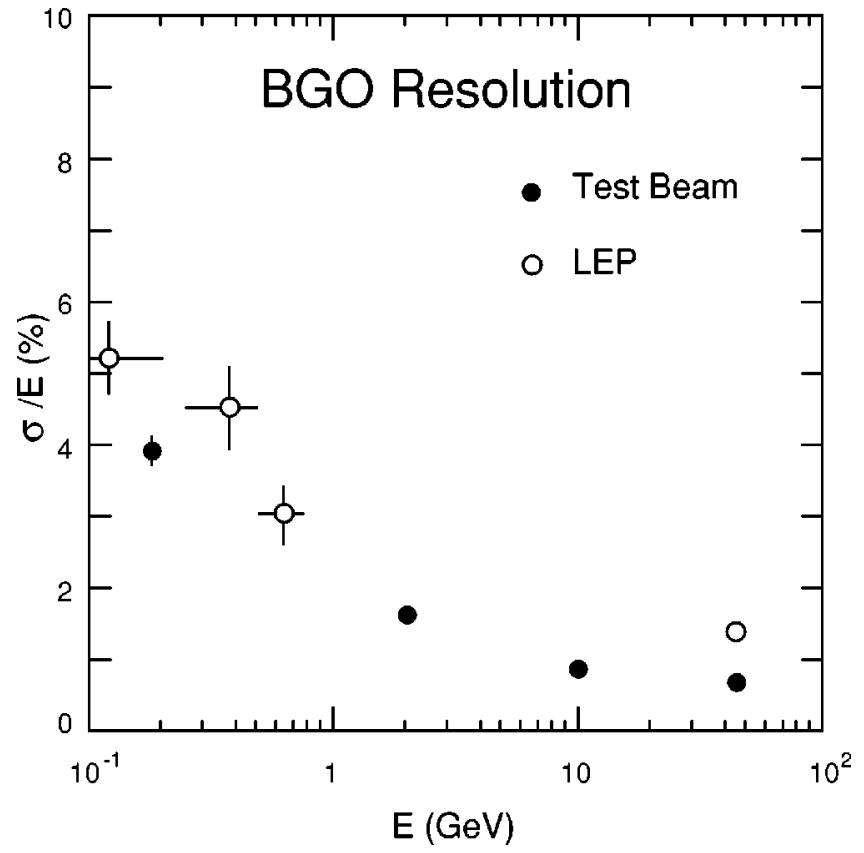

FIG. 7. Energy resolution of the L3 electromagnetic calorimeter as a function of energy, as obtained with test beam electrons (closed circles) and with physics data at LEP (open circles). From Karyotakis (1995). 


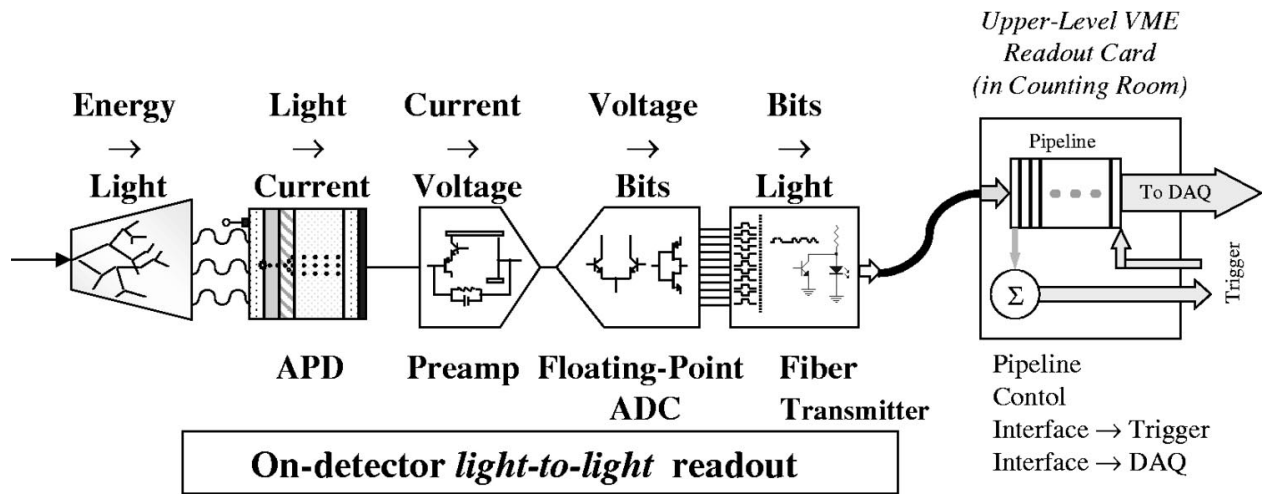

FIG. 8. The readout chain of the CMS electromagnetic calorimeter.

CMS goal is to achieve an output of $\sim 4000$ photoelectrons per GeV. Because of these small signals, and because of other stringent requirements for operation at the LHC (see Sec. IV.A), the CMS electromagnetic calorimeter is read out with a challenging and sophisticated almost fully digital electronic chain, shown in Fig. 8. The light signal from the crystal is transformed into an electric signal using avalanche photodiodes in the barrel part of the detector. These devices are $p-n$ junctions where photoelectrons undergo avalanche multiplication. Their gain $(\sim 50)$ is needed because of the small $\mathrm{PbWO}_{4}$ light yield. They are also able to work in the 4-T field in which the CMS calorimeter is immersed. The main drawback is their sensitivity to temperature ( $-2 \%$ gain variation per degree) and to the applied bias voltage $(-2 \%$ gain variation per volt), which requires temperature regulation and voltage control to better than 0.1 degrees and $40 \mathrm{mV}$, respectively. The avalanche photodiode is followed by a four-gain preamplifier-shaper system (shaping time $40 \mathrm{~ns}$ ) and by a 40-MHz 12-bit ADC. The ADC output is transformed back into light and transferred from the detector to the counting room by digital optical links at a rate of 800 $\mathrm{Mbit} / \mathrm{s}$. It is noteworthy that the detector-mounted readout chain, i.e., all components up to the ADC (see Fig. 8 ) plus the temperature regulation system, takes a radial space of $\simeq 25 \mathrm{~cm}$, which is comparable to the crystal thickness. The energy resolution obtained from the beam tests of a matrix of crystals is shown in Fig. 9. The data can be fit with a stochastic term of $3.3 \% / \sqrt{E(\mathrm{GeV})}$, a local constant term of $0.27 \%$, and a noise term of $0.19 / E(\mathrm{GeV})$. One of the main challenges of the CMS calorimeter is to achieve an overall constant term of the energy resolution of $\simeq 0.5 \%$, over the full detector acceptance, as needed at the LHC (see Sec. IV.A). In this respect, two effects are particularly important. Radiation damage, which affects the transparency of the $\mathrm{PbWO}_{4}$ crystals, is expected to produce a response drop of $\simeq 2 \%$ over the duration of an LHC run $(\simeq 15 \mathrm{~h})$. This drop can give rise to nonuniformities because it is not constant along the crystal depth (the front part of the crystal is more damaged by radiation), and the shower longitudinal profile fluctuates event by event. The full response can be recovered with a time constant of a few hours. In addition, as is also the case for the avalanche photodiode, the crystal response has a temperature dependence $(-2 \%$ light variation per degree at room temperature), which again requires temperature regulation and monitoring to better than $0.1{ }^{\circ} \mathrm{C}$. These effects can be controlled mainly by means of a laser system, by calibrating part of the crystals with test beams before installation in the final detector, and by using control physics samples (e.g., $Z \rightarrow e e$ events) when running at the LHC (see Sec. IV.C).

\section{d. Noble-liquid calorimeters}

The main features of noble liquids used for calorimetry applications ( $\mathrm{Ar}, \mathrm{Kr}, \mathrm{Xe})$ are presented in Table II. When a charged particle traverses these materials, about half of the lost energy is converted into ionization and half into scintillation. This latter is due to the recombination of electron-ion pairs, and gives rise to fast signals $(\sim 10 \mathrm{~ns})$ in the spectral region $120-170 \mathrm{~nm}$. The best energy resolution would obviously be obtained by collecting both the charge and light signal, which are anticorrelated as shown in Fig. 10. However, no large-scale

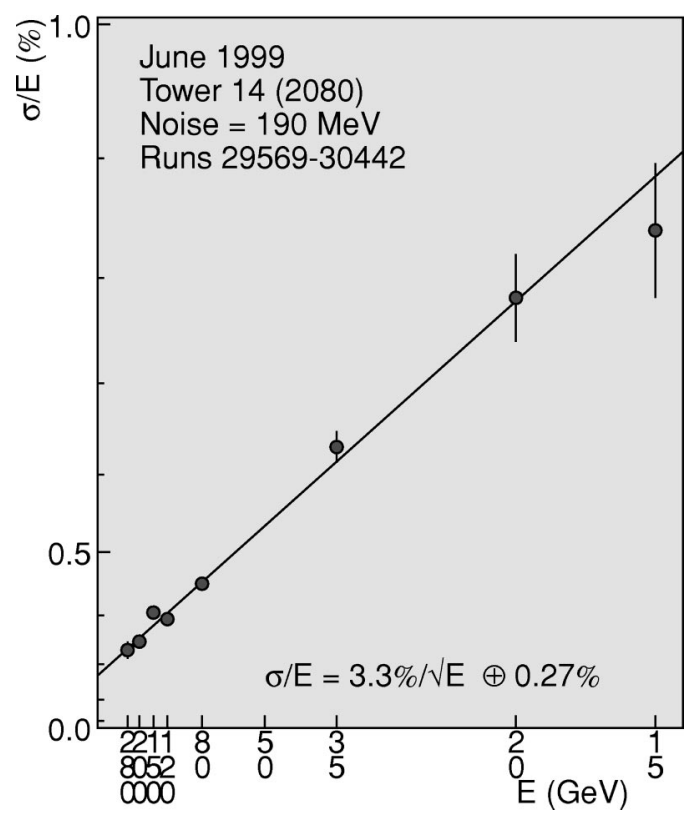

FIG. 9. The fractional energy resolution as a function of energy measured with electrons incident on a matrix of preseries crystals of the CMS electromagnetic calorimeter. The line is a fit with the function given in the plot. 
TABLE II. Main properties of liquid argon, krypton, and xenon.

\begin{tabular}{lccc}
\hline \hline & $\mathrm{Ar}$ & $\mathrm{Kr}$ & $\mathrm{Xe}$ \\
\hline$Z$ & 18 & 36 & 58 \\
$A$ & 40 & 84 & 131 \\
$X_{0}(\mathrm{~cm})$ & 14 & 4.7 & 2.8 \\
$R_{M}(\mathrm{~cm})$ & 7.2 & 4.7 & 4.2 \\
Density $\left(\mathrm{g} / \mathrm{cm}^{3}\right)$ & 1.4 & 2.5 & 3.0 \\
Ionization energy $(\mathrm{eV} /$ pair $)$ & 23.3 & 20.5 & 15.6 \\
Critical energy $\epsilon(\mathrm{MeV})$ & 41.7 & 21.5 & 14.5 \\
Drift velocity at saturation $(\mathrm{mm} / \mu \mathrm{s})$ & 10 & 5 & 3 \\
\hline \hline
\end{tabular}

calorimeter based on both readout principles has been constructed yet because of the technical and geometrical difficulties of extracting charge and light in the same instrument.

Excellent energy resolution can nevertheless be achieved in homogeneous liquid calorimeters by collecting the ionization signal alone, as demonstrated by the following simple calculation. If we assume that the full particle energy is absorbed by the liquid, and converted only into scintillation and ionization, then the total signal is given by $N=N_{\text {ion }}+N_{\text {scint }}$, where $N_{\text {ion }}$ is the number of electron-ion pairs and $N_{\text {scint }}$ the number of photons. The fluctuation on $N_{\text {ion }}$, i.e., the part of the signal which is read out, is (from binomial statistics)

$$
\sigma\left(N_{\text {ion }}\right)=\sqrt{N \frac{N_{\text {ion }}}{N} \frac{N_{\text {scint }}}{N}} .
$$

If, for instance, $80 \%$ of the released energy goes into ionization, i.e., $N_{\text {ion }} / N=0.8$, and $20 \%$ into scintillation, then Eq. (19) gives $\sigma\left(N_{\text {ion }}\right) \sim 0.4 \sqrt{N}$. Thus the energy resolution is a factor 2.5 better than that expected from a pure $1 / \sqrt{N}$ behavior (Fano factor).

Liquid argon is the most commonly employed noble liquid for sampling calorimeters (see Sec. II.C.2) be-

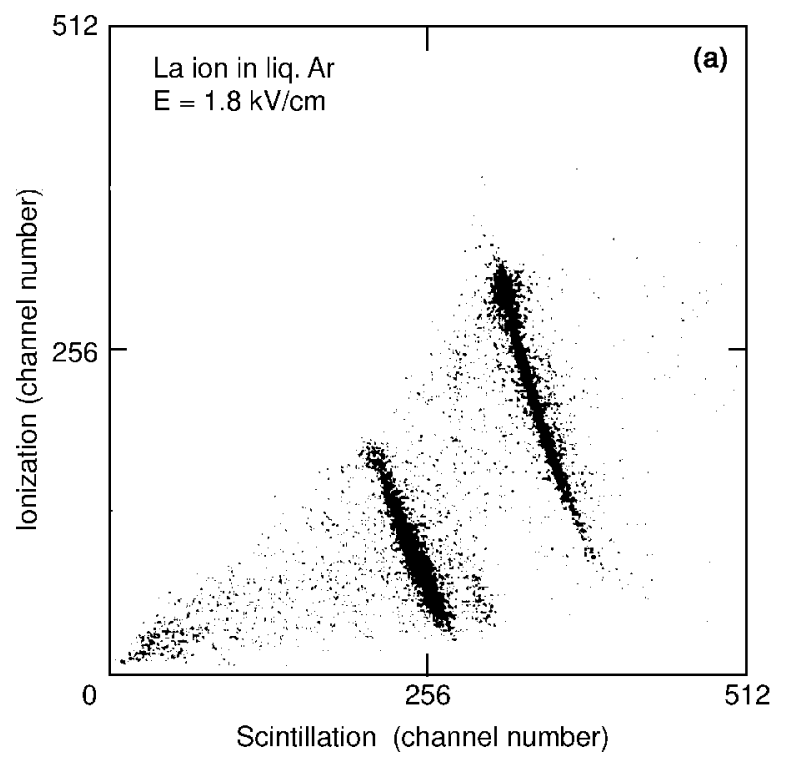

cause of its low cost and high purity. On the other hand, krypton is usually preferred for homogeneous calorimeters, mainly because of its much shorter radiation length, which allows more compact detectors. Xenon would be an even better choice in this respect; however, it is very rare in nature and therefore expensive. In general, noble-liquid calorimeters offer good radiation resistance and good response uniformity by construction since the liquid is distributed in a uniform way throughout the detector. The disadvantage of this technique is that it requires cryogenics and purification equipment.

An example of a homogeneous liquid calorimeter is the liquid-krypton electromagnetic calorimeter of the NA48 experiment (Barr et al., 1990; Fanti et al., 1999) at the CERN SPS. The aim of this fixed-target experiment was to measure the direct $C P$ violation parameter $\epsilon^{\prime} / \epsilon$ by detecting simultaneously $K_{S, L}^{0} \rightarrow \pi^{0} \pi^{0}$ and $K_{S, L}^{0}$ $\rightarrow \pi^{+} \pi^{-}$decays. The requirements for the electromagnetic calorimeter of such an experiment are numerous. A $\pi^{0} \rightarrow \gamma \gamma$ mass resolution of $1 \mathrm{MeV}$ is needed in order to reject the combinatorial background from $K_{L}^{0} \rightarrow 3 \pi^{0}$ when two photons are lost. This in turn requires an energy resolution of $\sim 5 \% / \sqrt{E(\mathrm{GeV})}$ and a position resolution of $1 \mathrm{~mm}$ for photons with $E \sim 25 \mathrm{GeV}$. The tagging of $K_{S}^{0}$ neutral decays by demanding a coincidence between the calorimeter signal and a beam hodoscope requires a calorimeter time resolution of better than 500 ps. In addition, the calorimeter must have a fast response in order to avoid event pileup, since the event rate was $\sim 100 \mathrm{kHz}$. The chosen technique is a quasihomogeneous liquid-krypton detector (Unal, 2001) schematically shown in Fig. 11. It has a length of $\simeq 1.2 \mathrm{~m}$ $\left(\simeq 25 X_{0}\right)$ and is segmented into 13500 cells of transverse size $2 \times 2 \mathrm{~cm}^{2}$ with no longitudinal segmentation. The readout electrodes run parallel to the beam direction and have a zigzag shape, so that the collected signal is independent of the shower distance from the electrodes. This is needed to achieve a good response uni-

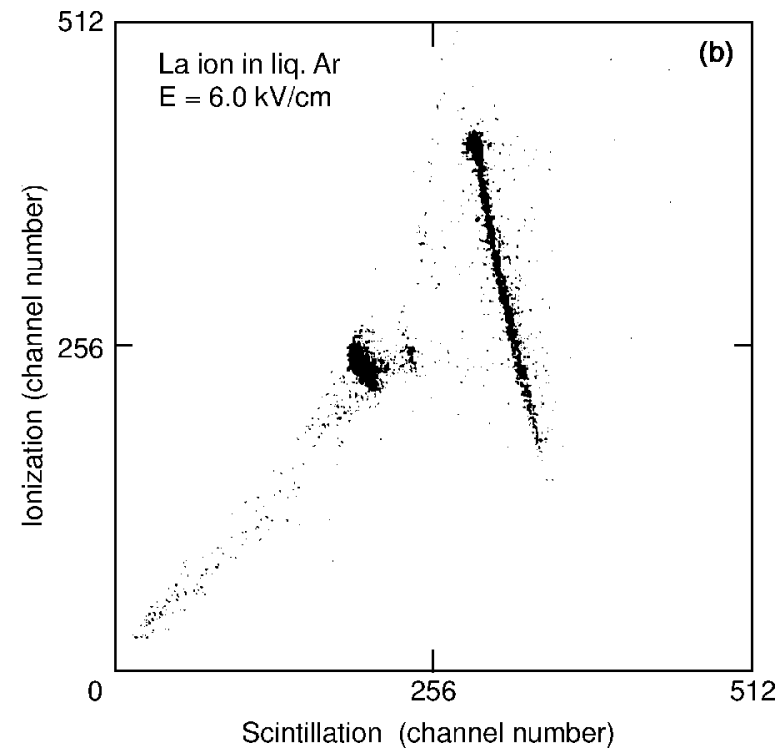

FIG. 10. The anticorrelation between the ionization signal and the scintillation signal produced in a liquid-argon chamber by a beam of La ions, for two values of the high voltage. From Crawford (1987). 
LKr CALORIMETER ELECTRODE STRUCTURE

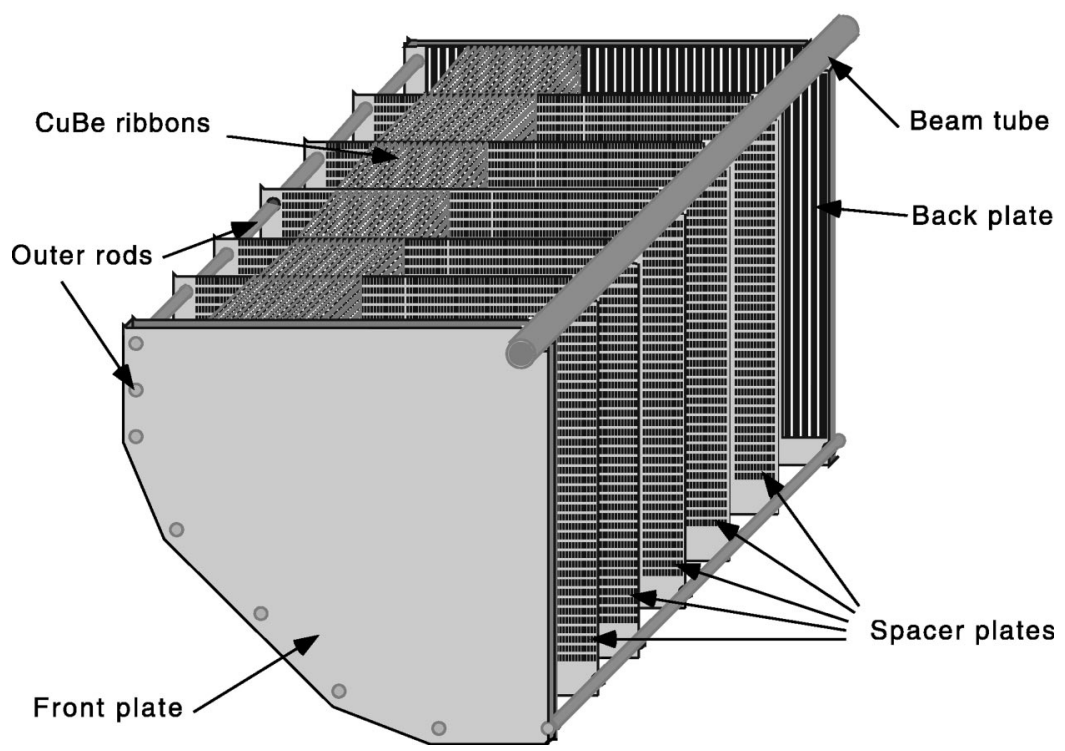

FIG. 11. Schematic view of a quarter of the NA48 liquid krypton electromagnetic calorimeter.

formity and a constant term of $\simeq 0.5 \%$. The calorimeter is read out with Si preamplifiers located on the detector faces, which therefore operate at the liquid krypton temperature of $120 \mathrm{~K}$, followed, outside the cryostat, by shapers (shaping time $80 \mathrm{~ns}$ ), 40-MHz ADC, and pipelines. Examples of the achieved performance with data (Martini, 1998) are shown in Fig. 12. The reconstructed $\pi^{0}$ mass has a resolution of $\simeq 1.1 \mathrm{MeV}$, and the calorimeter time resolution for $K^{0}$ events is $\simeq 230$ ps.

\section{Sampling calorimeters}

We have mentioned that the energy resolution of sampling calorimeters is in general worse than that of homogenous calorimeters, owing to the sampling fluctuations produced by the absorber layers interleaved with the active layers. It is typically in the range $5-20 \% / \sqrt{E(\mathrm{GeV})}$ for electromagnetic calorimeters.
On the other hand, sampling calorimeters are relatively easy to segment longitudinally and laterally, and therefore they usually offer better space resolution and particle identification than homogeneous detectors. They are almost universally used at accelerators to measure hadronic showers (see Sec. III), since they provide enough interaction lengths with a reasonable detector thickness (typically $<2 \mathrm{~m}$ ). They can be made compensated (see Sec. III.A). Moreover, the hadronic energy resolution is limited by the nature of strong interactions and not necessarily by sampling fluctuations.

Sampling calorimeters can be classified, according to the type of active medium, into scintillation calorimeters, gas calorimeters, solid-state calorimeters, and liquid calorimeters. In the first case the signal is collected in the form of light, in the last three cases in the form of electric charge. Commonly used absorber materials are lead, iron, copper, and uranium.
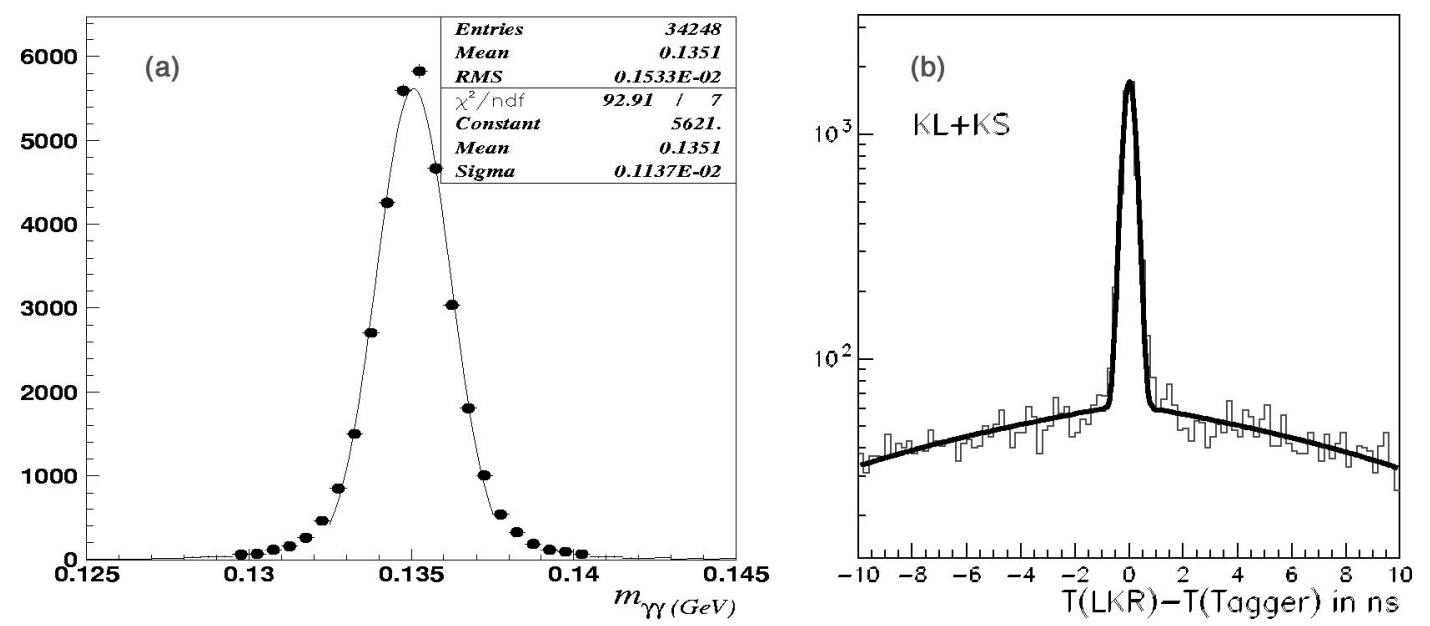

FIG. 12. (a) Reconstructed $\pi^{0}$ mass in the NA48 calorimeter. (b) The difference between the time measured by the NA48 calorimeter and the time given by a $K_{S}^{0}$ beam tagger. A peak due to $K_{S}^{0}$ events is visible on top of the background due to $K_{L}^{0}$ events. From Martini (1998). 


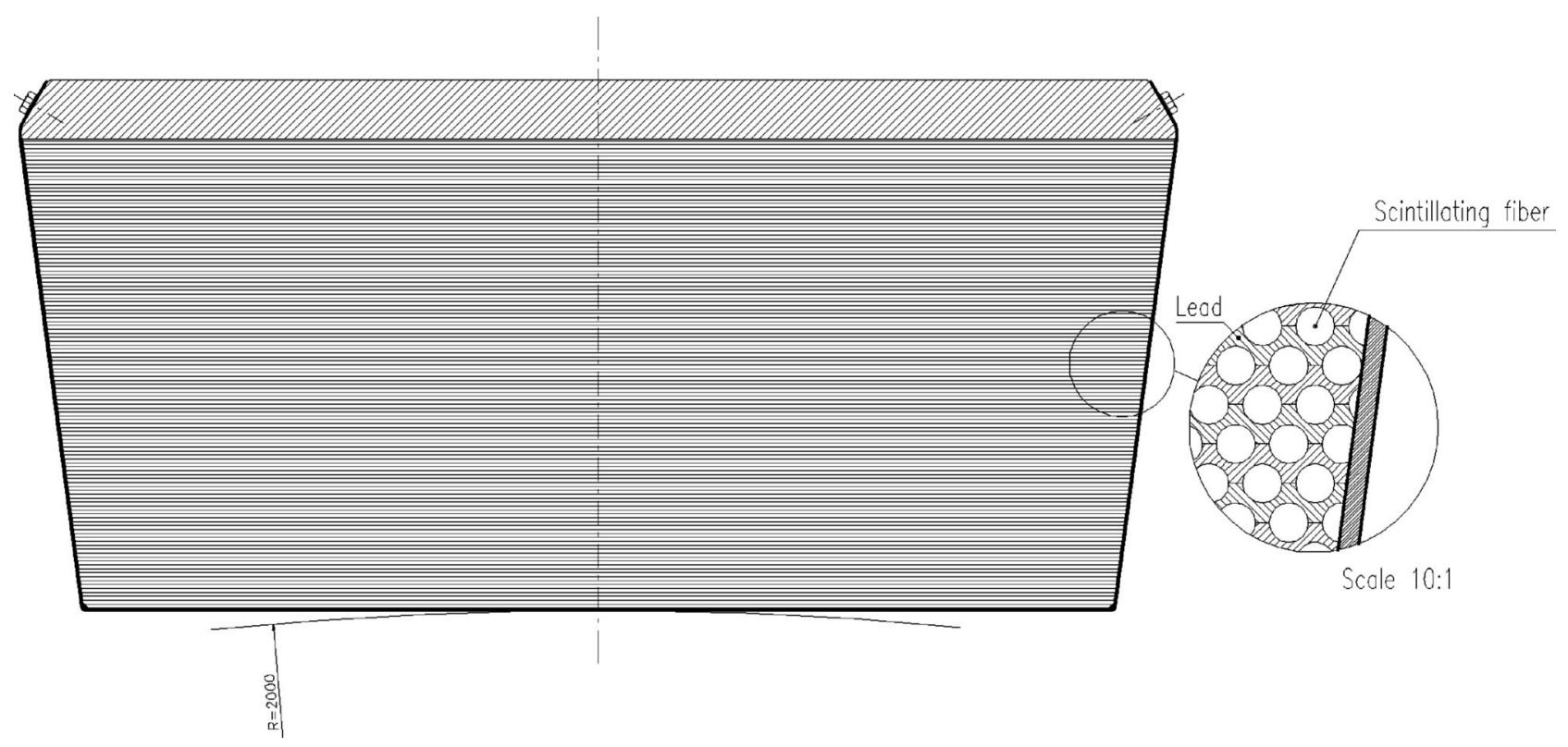

FIG. 13. Schematic layout of the barrel part of the KLOE electromagnetic calorimeter (Antonelli et al., 1995).

\section{a. Scintillation sampling calorimeters}

A large number of sampling calorimeters use organic (plastic) scintillators arranged in fibers or plates. These detectors are relatively cheap, can be built in a large variety of geometries, can be easily segmented, have a fast response and an acceptable light yield, and can be made compensating by properly tuning the ratio between the amounts of absorber and scintillator. Scintillation sampling calorimeters are used for instance in the ZEUS (Behrens et al., 1990) (see Sec. III.D), CDF (Balka et al., 1988; Bertolucci et al., 1988), and KLOE (Antonelli et al., 1995; Adinolfi et al., 2002) experiments.

The main drawback of this technique is that the optical readout suffers from aging and radiation damage. Furthermore nonuniformities at various stages of the light collection chain are often the source of a large constant term.

A more extensive discussion of these calorimeters can be found in Wigmans (2000). Here we describe briefly, as an example, the electromagnetic calorimeter of the KLOE experiment at DAФNE, the Frascati $\phi$ factory. The experimental goals (primarily the measurement of $C P$ violation in $K^{0}$ decays) and requirements are similar to those discussed in Sec. II.C.1 for the NA48 experiment. The calorimeter must reconstruct $K_{L, S}^{0} \rightarrow \pi^{0} \pi^{0}$ decays, which yield photons in the energy range 20-280 MeV, while rejecting the $K_{L}^{0} \rightarrow 3 \pi^{0}$ background. In addition, excellent time resolution is required in order to determine the $K^{0} \rightarrow \pi^{0}$ s vertex from the photon arrival times in the calorimeter. The chosen detector is a lead-scintillator calorimeter, consisting of $0.5-\mathrm{mm} \mathrm{Pb}$ layers in which 1-mm-diameter fibers are embedded. The calorimeter is divided into a barrel part and two end caps. The detector structure in the barrel is shown in Fig. 13. The $\sim 4$-m-long fibers run mostly orthogonal to the incident particles (they are parallel to the beam axis in the barrel) in order to avoid channeling, and are read out at both ends. The calorimeter thickness is $23 \mathrm{~cm}$, corresponding to about $15 X_{0}$. The readout granularity is $4.4 \mathrm{~cm}$ in the coordinate perpendicular to the fiber direction and $4.4 \mathrm{~cm}$ in depth. Because of the very thin absorber layers, the KLOE calorimeter is almost a homogeneous calorimeter. Beam tests, as well as measurements with complete physics events in the experiment, have shown an excellent energy resolution of $\sigma / E$ $\simeq 5 \% / \sqrt{E(\mathrm{GeV})}$ in the energy range $50-300 \mathrm{MeV}$. The reconstructed $\pi^{0} \rightarrow \gamma \gamma$ and $\eta \rightarrow \gamma \gamma$ mass spectra in $\phi$ $\rightarrow \pi^{+} \pi^{-} \pi^{0}$ and $\phi \rightarrow \eta \gamma$ events, respectively, are shown in Fig. 14. The optical properties of the selected fibers, e.g., the long attenuation length $(\sim 3 \mathrm{~m})$ and the large light yield, allow a time resolution of $\sigma_{t}$ $=54 \mathrm{ps} / \sqrt{E(\mathrm{GeV})} \oplus 50 \mathrm{ps}$, as measured with data.

\section{b. Gas sampling calorimeters}

Gas calorimeters have been widely employed until very recently (e.g., for LEP experiments), mainly because of their low cost and segmentation flexibility. However, they are not well suited to present and future machines because of their modest electromagnetic energy resolution $[\leqslant 20 \% / \sqrt{E(\mathrm{GeV})}]$, to which several effects, such as Landau fluctuations and path length variations in the active layers (Fischer, 1978), contribute. Owing to the low density of the active medium, the sampling fraction is small $(\ll 1 \%)$; operation in proportional mode is therefore required to obtain an acceptable signal-to-noise ratio. The operation of a gas calorimeter in proportional mode, i.e., with proportional wire planes in the active layers and a large voltage on the wires to produce avalanche multiplication of the electron signal, yields signal gains of $10^{3}-10^{5}$. However, the stability and uniformity of the detector response are modest, because the gain is very sensitive to several factors such as the precise diameter and position of the 

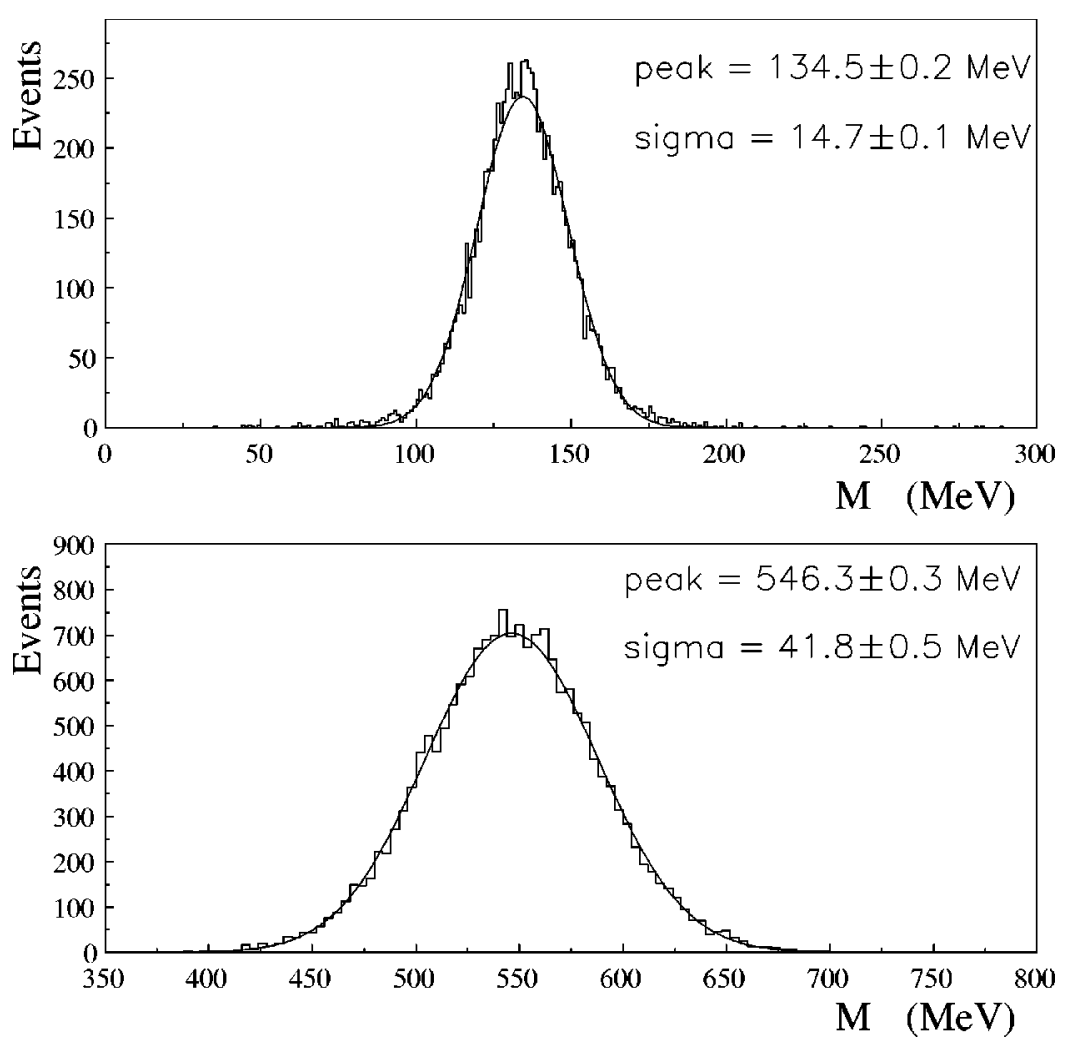

FIG. 14. Invariant mass distributions of photon pairs from $\phi \rightarrow \pi^{+} \pi^{-} \pi^{0}$ events with $\pi^{0}$ $\rightarrow \gamma \gamma$ (top) and from $\phi \rightarrow \eta \gamma$ events with $\eta$ $\rightarrow \gamma \gamma$ (bottom), as reconstructed in the KLOE calorimeter (Antonelli et al., 1995; Adinolfi et al., 2002). wires, the gas pressure, the temperature and purity, the high voltage setting, etc. It is difficult with this technique to achieve a response stability and uniformity at the level of a few permil, as is needed, for instance, at the LHC. One of the best performing gas sampling electromagnetic calorimeters was used in the ALEPH experiment at LEP (Décamp et al., 1990).

\section{c. Solid-state sampling calorimeters}

In most cases of solid-state sampling calorimeters the active medium is silicon. The main advantage of these detectors is that the density of the active layers is a factor of about 1000 larger than in gas calorimeters, which allows the construction of more compact devices and a higher signal-to-noise ratio. This latter is also due to the fact that only $3.6 \mathrm{eV}$ are needed to produce an electronhole pair in $\mathrm{Si}$, compared to $\simeq 30 \mathrm{eV}$ in gas. Therefore solid-state calorimeters are operated with unity gain, which avoids the drawbacks of charge multiplication (see above). The main disadvantages of this technique are the high cost, which prevents its use in large-scale detectors, and the poor radiation resistance. Small and compact Si sampling calorimeters, often employing a very dense absorber like tungsten, have been widely used as luminosity monitors for the LEP detectors.

\section{d. Liquid sampling calorimeters}

These detectors are discussed here in some detail because they offer good application perspectives for future experiments.

Warm-liquid (e.g., tetramethylpentane or TMP) calorimeters work at room temperature, without the over- head of cryogenics, which can be an advantage in certain specialized applications (Engler et al., 1999). However, they are characterized by poor radiation resistance and they suffer from purity problems.

Cryogenic liquid sampling calorimeters have been and still are widely employed in high-energy physics experiments (e.g., R807/ISR, Mark II, Cello, NA31, Helios, SLD, D0, H1), mainly with argon as the active medium. This well-established technique offers several advantages. The liquid density (see Table II) gives enough charge to allow operation in the ion chamber mode, which ensures a better response uniformity than in calorimeters with electron amplification. Liquid sampling calorimeters are relatively uniform and easy to calibrate because the active medium is homogeneously distributed inside the volume and the signal collection is not subject to the cell-to-cell variations that characterize detectors with optical readout. They provide good energy resolution $[\lesssim 10 \% / \sqrt{E(\mathrm{GeV})}]$ and a stable response with time. They are radiation hard. The drawbacks are the cryogenic equipment, which complicates the operation and introduces additional dead material in front of the calorimeter (cryostat); the need to achieve and maintain high-purity conditions, which in turn requires a purification system; and the fact that classical liquid calorimeters have a relatively slow charge collection. This last disadvantage, which would render these detectors unsuitable for operation at high-rate machines, has been recently overcome by the introduction of a novel geometry, the "accordion" geometry (Aubert, 1990), chosen for the ATLAS lead-liquid argon electromagnetic calorimeter. 


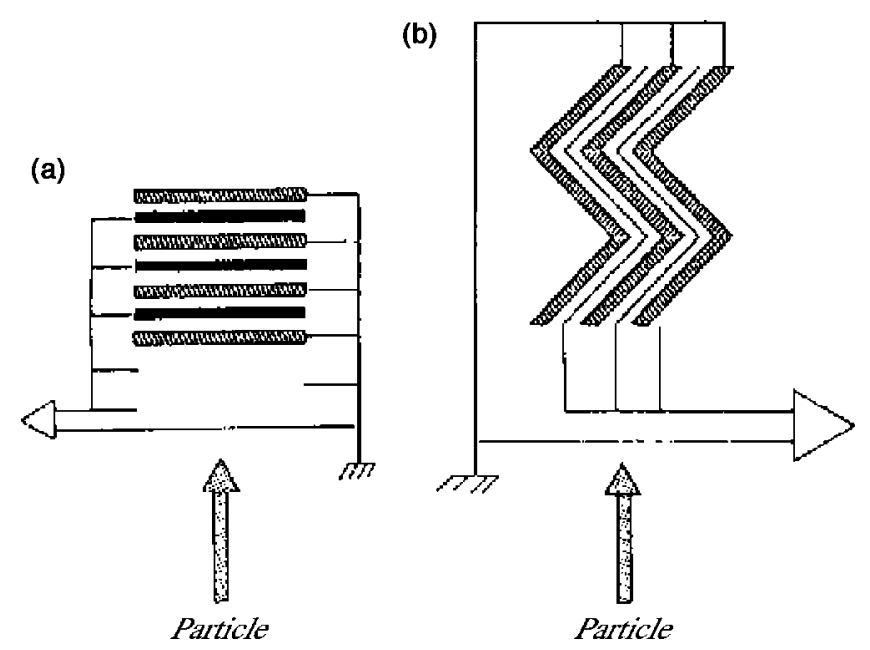

FIG. 15. Schematic view of a traditional sampling calorimeter geometry (a) and of the accordion calorimeter geometry (b).

In standard liquid-argon sampling calorimeters, the alternating absorber and active layers are disposed perpendicular to the direction of the incident particle, as illustrated in Fig. 15(a). The ionization signal produced by the shower in the liquid-argon gaps is collected by electrodes located in the middle of the gaps. These electrodes carry the high voltage, whereas the absorbers are at ground. For a typical liquid-argon gap of $2 \mathrm{~mm}$ on either side of the collection electrode and a high voltage of $\simeq 2 \mathrm{kV}$ across the gap, the electron drift time to the electrode is $\simeq 400 \mathrm{~ns}$. This time, which is needed to collect the total ionization charge, is too slow for operation at the LHC, where detector responses of $50 \mathrm{~ns}$ or smaller are needed (see Sec. IV.A). The solution is to integrate the ionization current over a time $\left(t_{p}\right)$ of only $40-50 \mathrm{~ns}$, thus collecting only a fraction of the total charge. This solution has the drawback that the signalto-noise ratio is degraded, and can only work if the signal transfer time from the electrodes to the readout chain is much smaller than $t_{p}$, i.e., if cables and connections (which introduce capacitance and inductance and therefore give rise to a long time constant of the circuit) are minimized. With the standard electrode geometry shown in Fig. 15(a), long cables are needed to gang together successive longitudinal layers to form calorimeter towers and to transfer the signal from these towers to the electronic chain, which is in general located at the end of one calorimeter module. As a consequence, the charge-transfer time from the electrodes to the first element of the readout chain (usually a preamplifier) is several tens of nanoseconds, i.e., comparable to $t_{p}$, and a very tiny signal is collected if $t_{p}=40-50 \mathrm{~ns}$. In addition, these cables introduce dead spaces between calorimeter towers at the expense of the detector hermeticity.

These problems can be solved by placing the absorber and gap layers perpendicular to the particle direction. In this way the signal from the collection electrodes can be extracted directly from the front and back faces of the calorimeter and sent to the readout chain with a minimum number of cables and connections. Dead spaces inside the detector active volume are also minimized

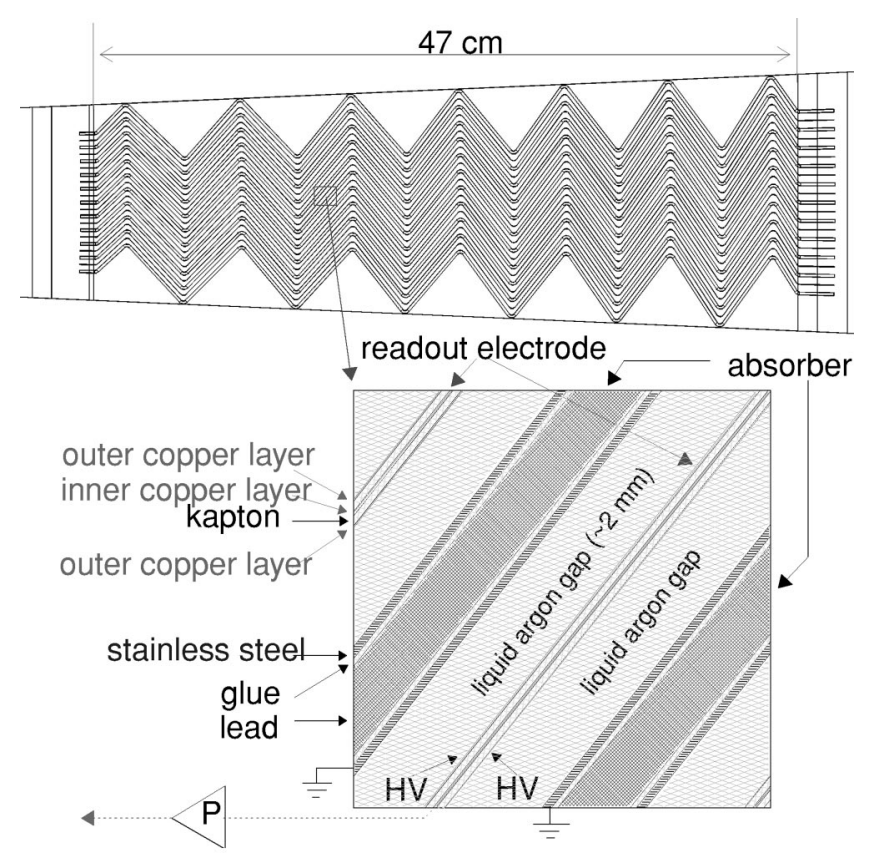

FIG. 16. Schematic view of the electrode structure of the ATLAS electromagnetic calorimeter. In the top picture particles enter the calorimeter from the left.

with this geometry. However, in order to prevent the incident particles from escaping through the liquidargon gaps without crossing the absorber, the electrodes must be bent into an accordion shape, as illustrated in Fig. 15(b).

This is the technique developed for the ATLAS electromagnetic calorimeter (ATLAS Collaboration, 1996b). The structure of this calorimeter is shown in Fig. 16. The lead layers have a thickness of 1.1-2.2 mm, depending on the rapidity region, and are separated by $4-\mathrm{mm}$ liquid-argon gaps. The calorimeter covers the rapidity region $|\eta|<3.2$ and is divided longitudinally into three compartments (Fig. 17). The first compartment is segmented into fine strips of pitch $4 \mathrm{~mm}$ in the $\eta$ direction, which provide good $\gamma / \pi^{0}$ separation capabilities as needed at the LHC; the second compartment has square towers of size $4 \times 4 \mathrm{~cm}^{2}$, and the third compartment has a factor of 2 coarser granularity in $\eta$ than the second compartment. The approximately 200000 channels are read out with a three-gain electronic chain located outside the cryostat and consisting of a preamplifier, a shaper (peaking time $\simeq 40 \mathrm{~ns}$ ), a $40-\mathrm{MHz}$ analog pipeline, and a 12-bit ADC. Digitization is performed in the last stage of the chain, after the first-level trigger has accepted an event.

Figure 18 shows two examples of expected performance. The energy resolution measured with a prototype module of the calorimeter is about $10 \% / \sqrt{E(\mathrm{GeV})}$, with a local constant term of $\simeq 0.3 \%$ and a noise term of $\simeq 0.25 / E(\mathrm{GeV})$ [Fig. 18(a)]. By using the longitudinal and lateral segmentation of the calorimeter, it will be possible to measure the direction of incident photons, and therefore the position of the primary vertex in $H \rightarrow \gamma \gamma$ events (see Sec. IV.A) with an estimated resolution of about $1 \mathrm{~cm}$ [Fig. 18(b)]. 


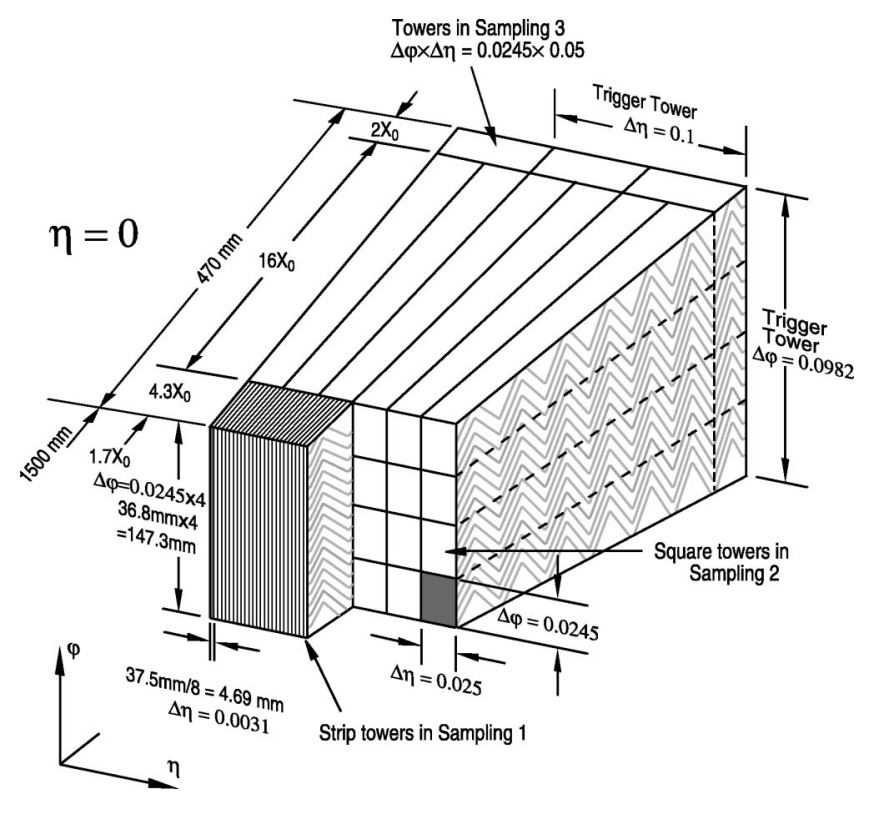

FIG. 17. Schematic view of the segmentation of the ATLAS electromagnetic calorimeter.

As already mentioned, one aspect that requires careful control is the purity of the liquid. Electronegative molecules dissolved in the liquid, such as oxygen molecules or unsaturated carbon composites, can capture the ionization electrons, thus reducing the collected signal. Therefore it is important to avoid any oxygen leaks inside the cryostat, and materials that emit impurities by outgassing (for instance, when exposed to high radiation doses). Argon is the best liquid from this point of view. It has the lowest boiling temperature ( $87 \mathrm{~K}$ compared to $120 \mathrm{~K}$ for krypton), and therefore outgassing is reduced. Commercial liquid argon is very pure (impurity concentration below $\simeq 0.5 \mathrm{ppm}$ ) whereas krypton needs purification. It is relatively cheap, and therefore can easily be

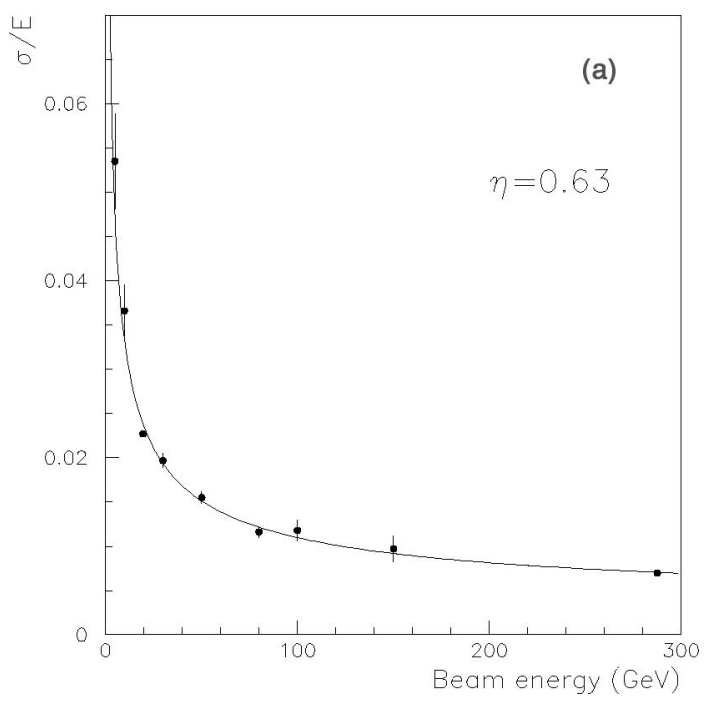

replaced in the event of major pollution, whereas krypton and xenon are more expensive. Experience with the $\mathrm{H} 1$ and D0 calorimeters, which have been equipped with sophisticated systems of probes and purity monitors, has shown a very good control of the liquid quality. For instance, the response of the D0 calorimeter has dropped by only $0.5 \%$ over ten years of operation. In addition, purity is less of an issue if the calorimeter is operated with a fast shaping time, because the electrons drift over only very short distances $\left(\simeq 200 \mu \mathrm{m}\right.$ for $\left.t_{p} \simeq 40 \mathrm{~ns}\right)$ before being collected. In the ATLAS calorimeter the sensitivity to impurities is reduced by a factor of $10 \mathrm{com}$ pared to that of the $\mathrm{H} 1$ and D0 calorimeters which have integration times of $\simeq 450 \mathrm{~ns}$.

On the other hand, when operating with fast shaping, the liquid response exhibits a quite strong temperature dependence and typically drops by $2 \%$ for a temperature increase of one degree. Care must therefore be taken to ensure a temperature distribution inside the cryostat uniform to a fraction of a degree.

Finally, it should be noted that the ATLAS and CMS experiments, although motivated by exactly the same physics goals, have chosen two completely different calorimeters, which demonstrates that often more than one solution exists for a given case. CMS has put the emphasis on excellent intrinsic energy resolution, hence the choice of crystals, whereas ATLAS has preferred a technique with moderate energy resolution but with potentially a more uniform response and with angular measurement and powerful particle identification capabilities. The readout chain is also different in the two calorimeters, i.e., almost fully digital in CMS and almost fully analog in ATLAS.

\section{HADRON CALORIMETRY}

In this section we present the physics and certain aspects of the detectors used in modern hadron calorim-

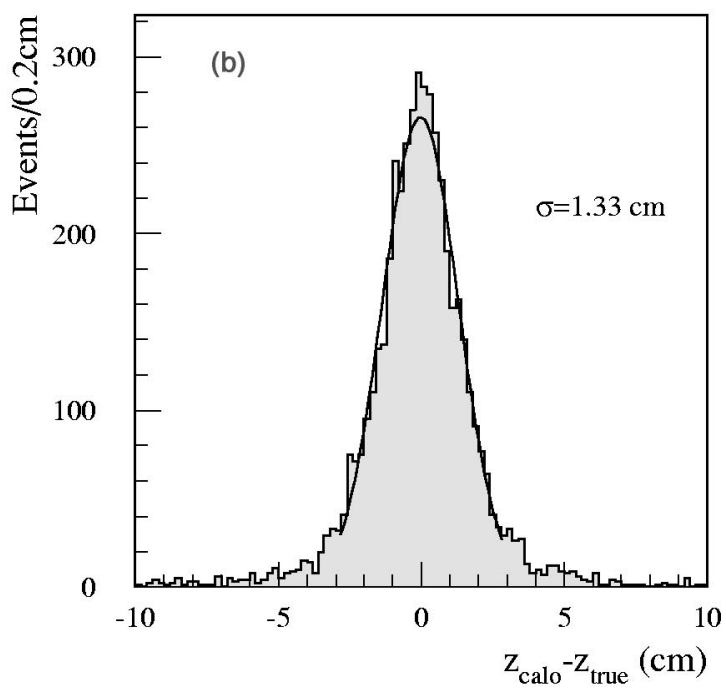

FIG. 18. (a) Fractional electron energy resolution as a function of energy as obtained from a beam test of the "module zero" of the ATLAS barrel electromagnetic calorimeter (the electronic noise has been subtracted). (b) Difference between the true primary vertex and the vertex reconstructed by the electromagnetic calorimeter, as obtained for $H \rightarrow \gamma \gamma$ events with $m_{H}$ $=100 \mathrm{GeV}$ simulated with GEANT. 
TABLE III. Characteristic properties of the hadronic cascade.

\begin{tabular}{|c|c|c|c|c|}
\hline Reaction & Properties & $\begin{array}{l}\text { Influence on energy } \\
\text { resolution }\end{array}$ & $\begin{array}{l}\text { Characteristic } \\
\text { time }(\mathrm{s})\end{array}$ & $\begin{array}{l}\text { Characteristic } \\
\text { length }\left(\mathrm{g} \mathrm{cm}^{-2}\right)\end{array}$ \\
\hline $\begin{array}{l}\text { Hadron } \\
\text { production }\end{array}$ & $\begin{array}{l}\text { Evaporation } \simeq A^{0.1} \ln \mathrm{s} \\
\text { Inelasticity } \simeq 1 / 2\end{array}$ & $\begin{array}{l}\pi^{0} / \pi^{+} \text {ratio } \\
\text { Binding energy loss }\end{array}$ & $10^{-22}$ & Abs. length $\lambda \simeq 35 A^{1 / 3} \mathrm{~g} \mathrm{~cm}^{-2}$ \\
\hline $\begin{array}{l}\text { Nuclear } \\
\text { deexcitation }\end{array}$ & $\begin{array}{l}\text { Evaporation energy } \simeq 10 \% \\
\text { Binding energy } \simeq 10 \% \\
\text { Fast neutrons } \simeq 40 \% \\
\text { Fast protons } \simeq 40 \%\end{array}$ & $\begin{array}{l}\text { Binding energy loss } \\
\text { Different response of } \\
\text { detecting medium to } n, \\
\text { charged particle, and } \gamma_{\text {s }}\end{array}$ & $10^{-18}-10^{-13}$ & $\begin{array}{l}\text { Fast neutrons } \lambda_{n} \simeq 100 \\
\text { Fast protons } \lambda_{p} \simeq 20\end{array}$ \\
\hline $\begin{array}{l}\text { Pion and } \\
\text { muon decays }\end{array}$ & $\begin{array}{l}\text { Fractional energy of } \mu \text { 's } \\
\text { and } \nu \text { 's } \simeq 5 \%\end{array}$ & Loss of $\nu$ 's & $10^{-8}-10^{-6}$ & $\gg \lambda$ \\
\hline $\begin{array}{l}\text { Decay of } c, b \\
\text { particles } \\
\text { produced in } \\
\text { multi-TeV } \\
\text { cascades }\end{array}$ & $\begin{array}{l}\text { Fractional energy of } \mu \text { 's } \\
\text { and } \nu \text { 's at percent level }\end{array}$ & $\begin{array}{l}\text { Loss of } \nu \text { 's, } \mu \text { 's } \\
\text { Tails in resolution } \\
\quad \text { function }\end{array}$ & $10^{-12}-10^{-10}$ & $\ll \lambda$ \\
\hline
\end{tabular}

etry. We emphasize the degree of understanding which has transformed the design of such instruments from an empirical art to a science-based, powerful, and widely used technology.

These devices were first employed in the study of the cosmic-ray spectrum during the late 1950s. The energy $E$ was assumed to be related to the hadronic shower multiplicity $n(x)$ of fast charged particles versus shower depth $x$ through the specific ionization $\epsilon$ as $E$ $=\epsilon \int n(x) d x$. This estimate, crude by modern standards, is nevertheless correct to within a factor of $\simeq 2$ (Murzin, 1967).

Modern instruments are built with a good understanding of the physics of the hadronic cascade (Sec. III.A) and of the limits to the energy resolution (Sec. III.B). The optimization of such detectors can be attained because the signal response of calorimeters to the shower particles is understood, also thanks to the development of Monte Carlo codes of the showering process (Sec. III.C). State-of-the art calorimeter facilities have been constructed (Sec. III.D) based on this knowledge.

\section{A. Physics of the hadronic cascade}

By analogy with electromagnetic showers, the energy degradation of hadrons proceeds through an increasing number of (mostly) strong interactions with the calorimeter material. However, the complexity of the hadronic and nuclear processes produces a multitude of effects that determine the functioning and the performance of practical instruments, and make hadronic calorimeters more complicated instruments to optimize. Experimental studies by many groups helped to unravel these effects and permitted the design of optimized detectors.

Some of the characteristic properties of the hadronic cascade are summarized in Table III. The hadronic interaction produces two classes of effects. First, energetic secondary hadrons are produced with a mean free path ("interaction length") $\lambda \approx 35 A^{1 / 3} \mathrm{~g} \mathrm{~cm}^{-2}$ between interactions; their momenta are typically a fair fraction of the primary hadron momentum, i.e., at the GeV scale. Sec- ond, in hadronic collisions with the material nuclei, a significant part of the primary energy is consumed in nuclear processes such as excitation, nucleon evaporation, spallation, etc., resulting in particles with characteristic nuclear energies at the $\mathrm{MeV}$ scale.

We first address features of particle production in the hadronic cascade. The richness of the physics is illustrated in Fig. 19, which shows the spectra of the major particle components, averaged over many cascades, induced by $100-\mathrm{GeV}$ protons in lead. These spectra are-in a spectacular fashion-dominated by electrons, positrons, photons, and neutrons at low energy. Note the structures in the photon spectrum: the line at approximately $8 \mathrm{MeV}$ is the result of an $(n, \gamma)$ reaction and a fingerprint of nuclear physics; the line at $511 \mathrm{keV}$ results from $e^{+} e^{-}$annihilation photons. These low-energy spectra encapsulate all the information relevant to the hadronic energy measurement. Deciphering this message becomes the story of hadronic calorimetry.

The energy dependence of one component is shown in Fig. 20. Neutrons are chosen because they are representative of other particles (protons, pions, etc.) at high energy and also a yardstick for the importance of nuclear effects. The ordinate-flux times energy-exhibits the approximate proportionality between the number of particles in the shower and the energy of the incident particle.

Figure 20 exhibits a small, albeit significant, deviation from a linear energy dependence. It is due to a fairly obvious physics feature, shown in Fig. 19. The fast hadronic component contains protons, neutrons, charged pions, and neutral pions. Because of the charge independence of hadronic interactions in each high-energy collision, on average one-third of the pions produced will be $\pi^{0}$ 's, $F_{\pi^{0}}=1 / 3$. This is the "odd man" in this group of energetic hadrons because these neutral pions will decay to two photons, $\pi^{0} \rightarrow \gamma \gamma$, before having a chance to reinteract hadronically. We have already analyzed how these photons behave: they will induce an electromagnetic cascade, proceeding along its own laws of electromagnetic interactions. This physics process acts like a 


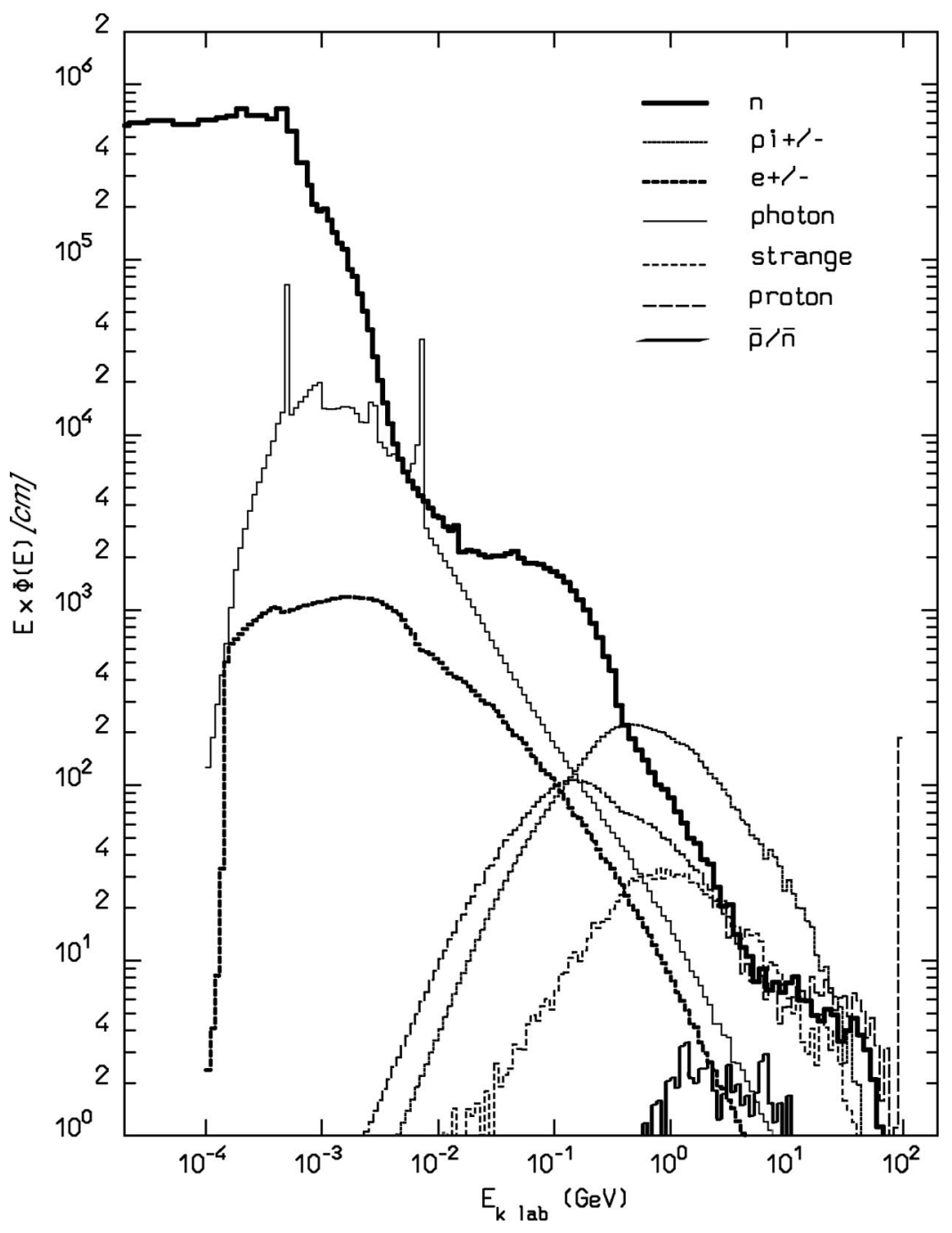

FIG. 19. Particle spectra produced in the hadronic cascade initiated by $100-\mathrm{GeV}$ protons absorbed in lead. The energetic component is dominated by pions, whereas the soft spectrum is composed of photons and neutrons. The ordinate is in "lethargic" units and represents the particle track length, differential in $\log E$. The integral of each curve gives the relative fluence of the particle. Fluka calculations (Ferrari, 2001). "one-way diode," transferring energy from the hadronic part to the electromagnetic component, which will not contribute further to hadronic processes.

As the number of energetic hadronic interactions increases with incident energy, so will the fraction of the electromagnetic cascade. This simple picture of the hadronic showering process leads to a power-law dependence of the two components [see also Gabriel et al. (1994) and Groom (1998)]; for the hadronic fraction $F_{h}$ one finds $F_{h}=\left(E / E_{0}\right)^{k}$ with $k=\ln \alpha / \ln m$. The parameter $E_{0}$ denotes a cutoff for further hadronic production, typically $E_{0} \approx 1-2 \mathrm{GeV} ; m$ is the multiplicity of fast hadrons produced in a hadronic collision; the parameter $\alpha$ gives the fraction of hadrons not decaying electromagnetically; the value of $k$ is $\approx-0.2$. Values of $F_{h}$ are of order $0.5(0.3)$ for a 100 (1000) -GeV shower. As the energy of the incident hadron increases, it is doomed to dissipate its energy in a flash of photons.

This identity change from hadronic to electromagnetic energy is reflected in the relative hadronic particle fluxes shown in Fig. 20. The relative reduction of neutrons is balanced by increased electromagnetic energy. More quantitatively, the various components are presented in Fig. 21 for protons on lead.
Contributions from neutrons and photons from nuclear reactions, which have consequences for the performance of these instruments, are also shown in Fig. 21. The total energy carried by photons from nuclear reactions is substantial: only a fraction, however, will be recorded in practical instruments, as most of these photons are emitted with a considerable time delay ( $\leqslant 1 \mu \mathrm{s})$. These delayed photons, soft neutrons, and binding energy all show that these nuclear effects produce a form of "invisible" energy. In general this invisible energy cannot be detected at all or only with much reduced efficiency. Let $\eta_{e}$ be the efficiency for observing a signal $E_{\text {vis }}^{e}$ (visible energy) from an electromagnetic shower, i.e., $E_{\mathrm{vis}}^{e}=\eta_{e} E(\mathrm{em})$; let $\eta_{h}$ be the corresponding efficiency for purely hadronic energy to provide visible energy in an instrument. Therefore for a pion-induced shower the visible energy $E_{\text {vis }}^{\pi}$ is

$$
\begin{aligned}
E_{\mathrm{vis}}^{\pi} & =\eta_{e} F_{\pi^{0}} E+\eta_{h} F_{h} E \\
& =\eta_{e}\left(F_{\pi^{0}}+\frac{\eta_{h}}{\eta_{e}} F_{h}\right) E,
\end{aligned}
$$




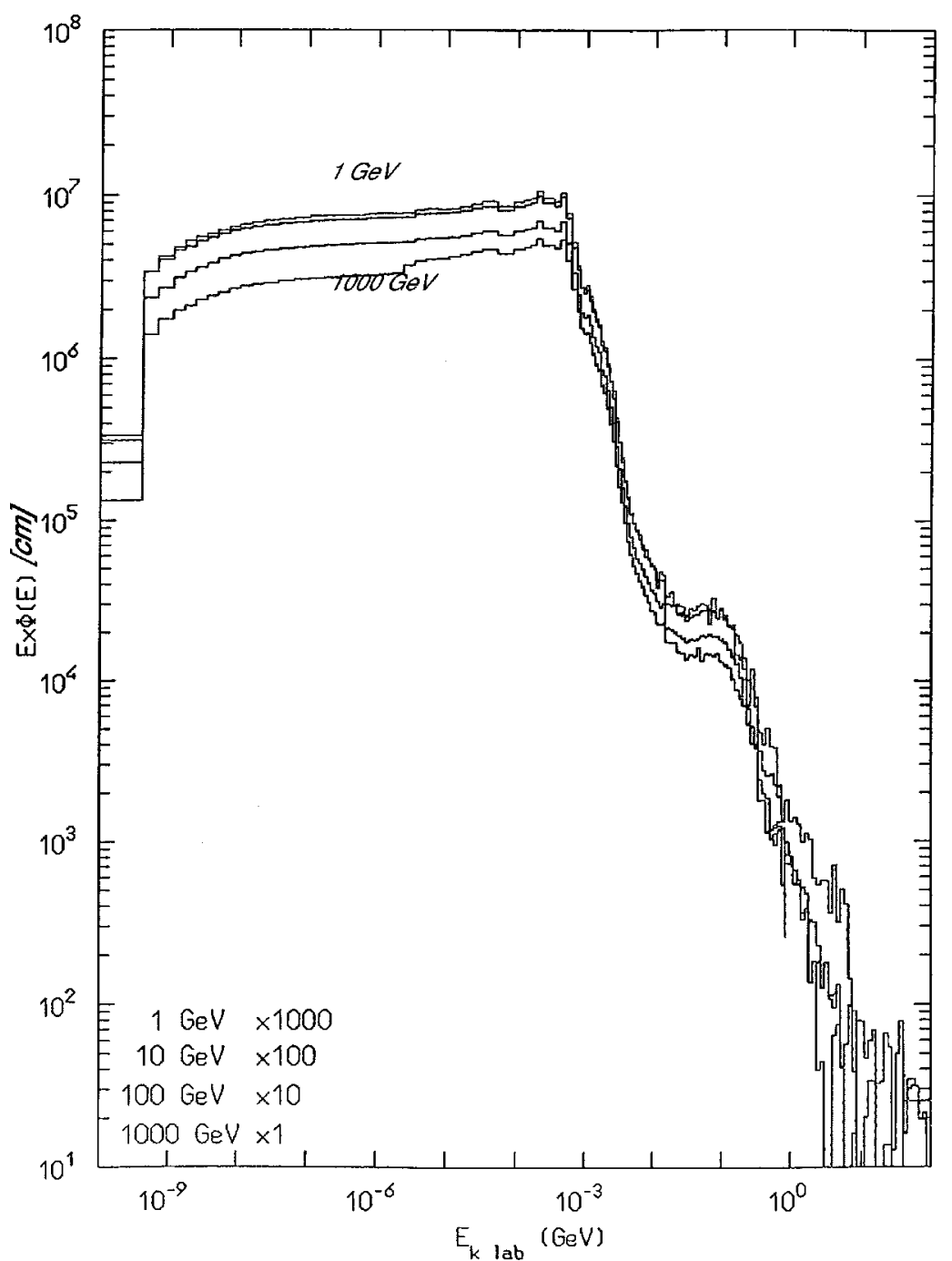

FIG. 20. The "universality" of the shower particle spectrum as a function of energy is only approximate. Shown is one componentneutrons-produced by protons on lead. With increasing energy of the incident hadron, the hadronic component is reduced relative to the electromagnetic component. The ordinate is as in Fig. 19 (Ferrari, 2001) where $E$ is the incident pion energy. The ratio of observable, i.e., "visible," signals induced by electromagnetic and hadronic showers, usually denoted $e / \pi$, is therefore

$$
\frac{E_{\mathrm{vis}}^{\pi}}{E_{\mathrm{vis}}^{e}}=\left(\frac{e}{\pi}\right)^{-1}=1-\left(1-\frac{\eta_{h}}{\eta_{e}}\right) F_{h} .
$$

In general $\eta_{e} \neq \eta_{h}$, therefore the average response of a hadron calorimeter as a function of energy will not be linear because $F_{h}$ decreases with incident energy. More subtly, for $\eta_{h} \neq \eta_{e}$ we have to expect that event-by-event fluctuations in the $F_{h}$ and $F_{\pi^{0}}$ components will have an impact on the energy resolution of such instruments. The relative response $e / \pi$ turns out to be the most important yardstick for gauging the performance of a hadronic calorimeter. In the following we show that fluctuations in the invisible energy dominate the fluctuations in the detector signal, and hence the energy resolution. The road to high-performance hadronic calorimetry has been opened by understanding how to compensate for these invisible energy fluctuations (Fabjan and Willis, 1975).

\section{B. Energy resolution of hadron calorimeters}

The average properties of the hadronic cascade also determine the intrinsic fluctuations, and hence the energy resolution, as we shall discuss. Subsequently we shall analyze further contributions due to features of practical detectors, including the signal response of their instrumentation.

Inescapably, hadronic cascades imply nuclear interactions with their correlated invisible energy. With less energy measurable from a hadronic shower than from an electromagnetic shower, we expect that on average for particles with the same incident energy the signal response to hadrons will be lower, i.e., $e / \pi>1$. Event by event the visible energy will fluctuate between two extremes: fully electromagnetic, yielding the same signal as an electron, or fully hadronic with a maximum of invisible energy, as shown conceptually in Fig. 22.

This simple analysis already provides the following qualitative conclusions for instruments with $e / \pi \neq 1$ :

- fluctuations in $F_{\pi^{0}}$ are a major component of the energy resolution;

- the average value $\left\langle F_{\pi^{0}}\right\rangle$ is energy dependent and therefore calorimeters have a response to hadrons that is nonlinear with energy; 


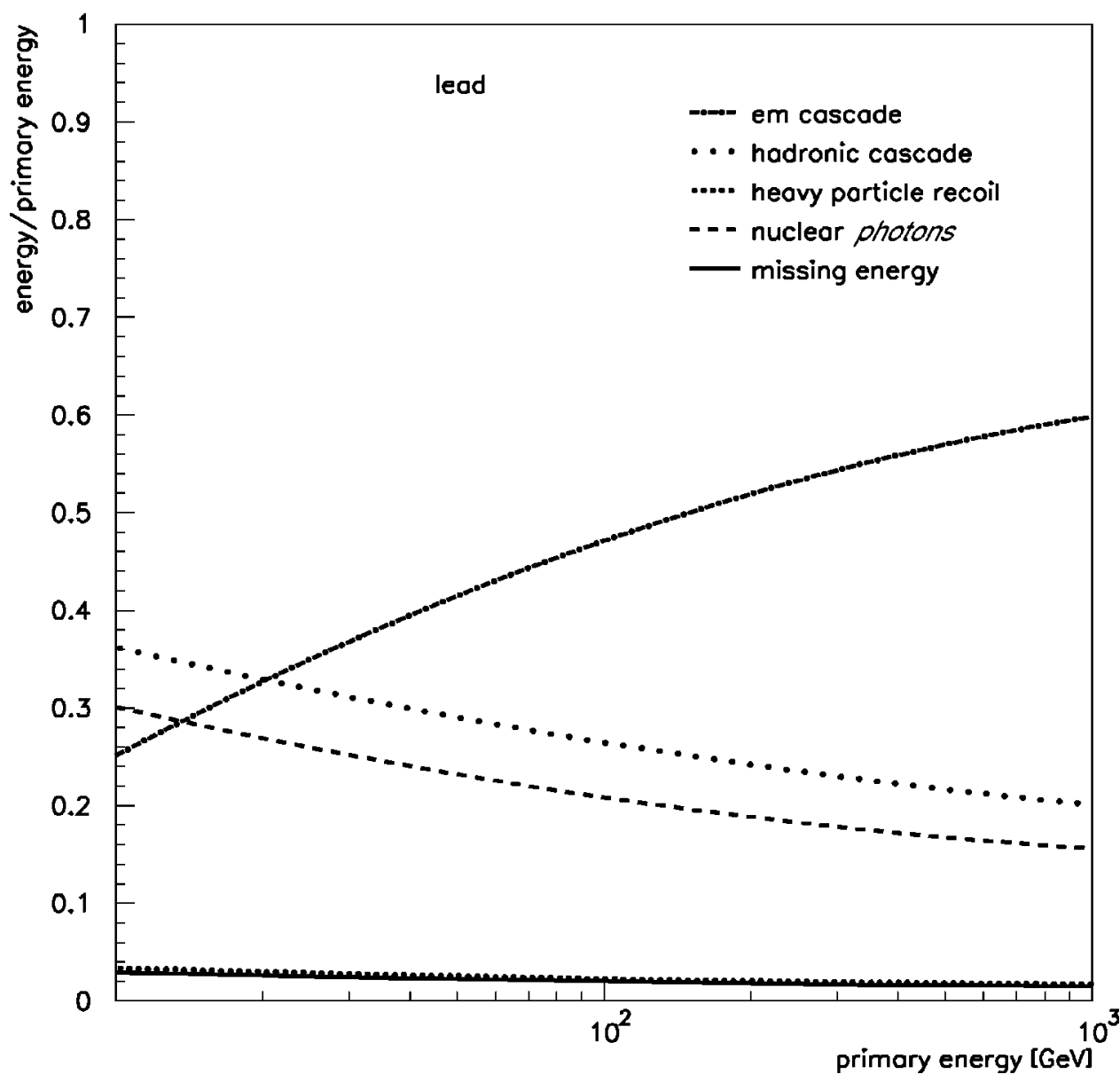

FIG. 21. Characteristic components of proton-initiated cascades in lead. With increasing primary energy the $\pi^{0}$ component increases (Ferrari, 2001).

- the above-mentioned fluctuations are nonGaussian and therefore the energy resolution scales weaker than $1 / \sqrt{E}$.

Detectors that achieve compensation for the loss of invisible energy, i.e., $e / \pi=1$, are called "compensated" calorimeters.

The effect of $e / \pi$ has been observed (Fig. 23), and evaluated (Wigmans, 1988) quantitatively (Fig. 24). Re-

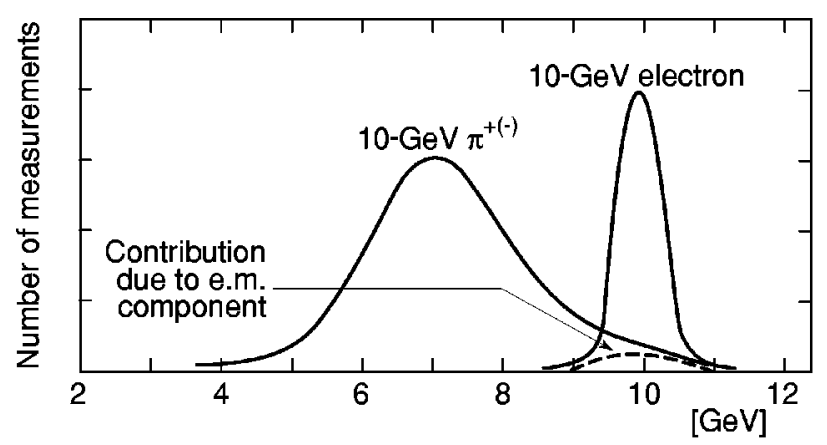

Signal (in energy units) obtained for a $10 \mathrm{GeV}$ energy deposit

FIG. 22. Conceptual response of calorimeters to electrons and hadrons. The curves are for an electromagnetic calorimeter with $\sigma / E=0.1 / \sqrt{E}$ and for a hadronic calorimeter with $\sigma / E$ $=0.5 / \sqrt{E}$ and $e / \pi=1.4$. The hadron-induced cascade fluctuates between almost completely electromagnetic and almost completely hadronic energy deposit, broadening the response and producing non-Gaussian tails. markably, it is possible to "tune" the $e / \pi$ response of a calorimeter in the quest for achieving $e / \pi=1$, and thus optimize the performance.

A convenient (albeit nontrivial) reference scale for the calorimeter response is the signal from minimumionizing particles (mip). We define $e /$ mip as the signal produced by an electron relative to a mip. Assume the case of a mip depositing, for example, $\alpha \mathrm{GeV}$ in a given calorimeter. If an electron depositing $\beta \mathrm{GeV}$ produces a signal $\beta / \alpha$, the instrument is characterized by a ratio $e / \operatorname{mip}=1$. Similarly, the relative response to the purely hadronic component of the hadron shower is $\eta_{h} F_{h} E / \mathrm{mip}$, or $h / \mathrm{mip}$. The latter can be decomposed into

$$
h / \operatorname{mip}=\left(\begin{array}{lll}
f_{\text {ion }} \operatorname{ion} / \operatorname{mip}+f_{n} & n / \operatorname{mip}+f_{\gamma} & \gamma / \mathrm{mip}
\end{array}\right) .
$$

The fractions $f_{\text {ion }}, f_{n}, f_{\gamma}$ denote the average fractions of ionizing hadrons, neutrons, and photons.

Practical hadron calorimeters are almost always built as sampling devices; the energy sampled in the active layers, $f_{\text {samp }}$ [Eq. (17)], is typically a small fraction, a few percent or less, of the total incident energy.

The energetic hadrons lose relatively little energy ( $\$ 10 \%$ ) through ionization before being degraded to such low energies that nuclear processes dominate. Therefore the response of the calorimeter will be dominated by the values of $n / \mathrm{mip}$ and $\gamma / \mathrm{mip}$ in both the absorber and the readout materials. 

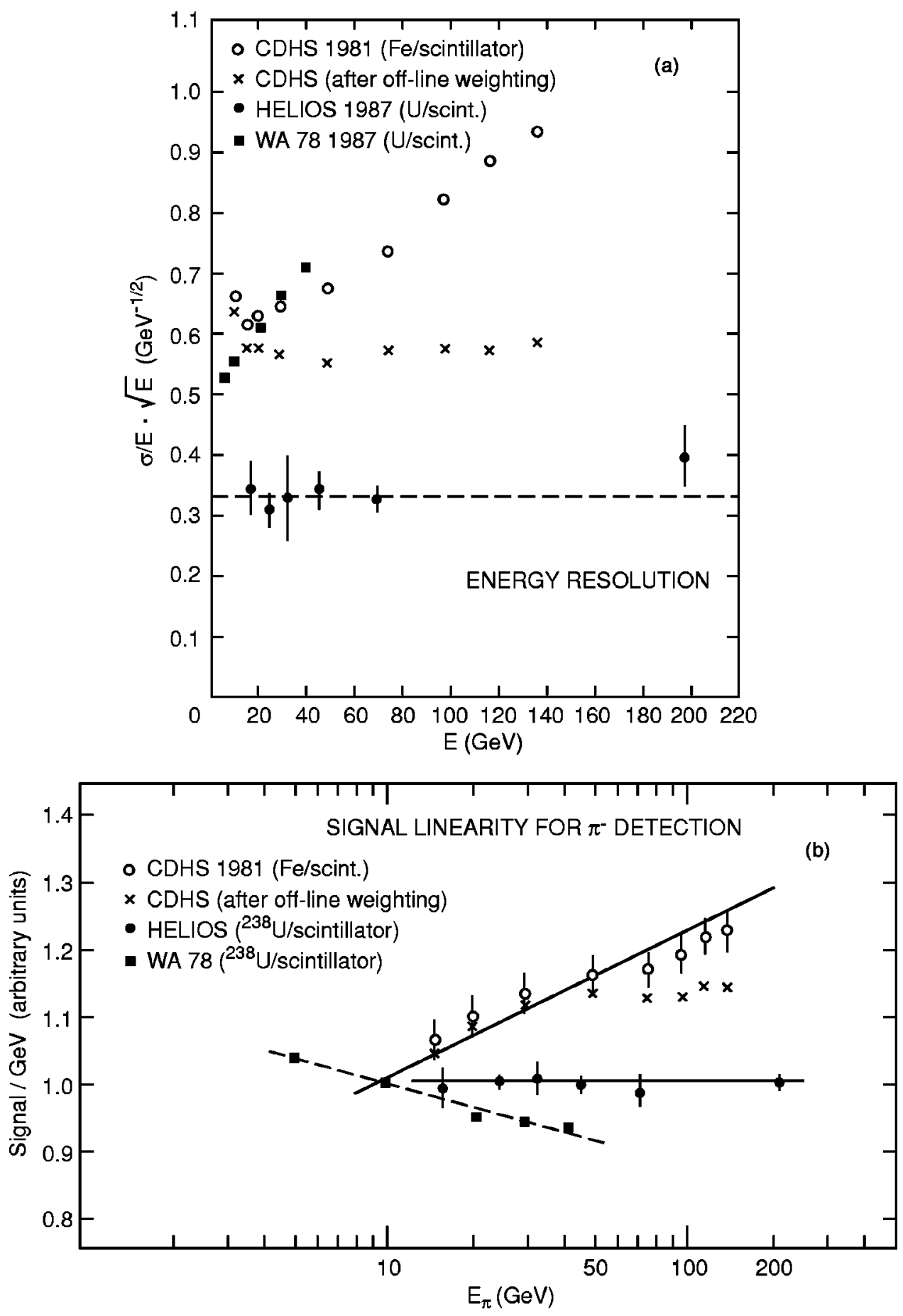

FIG. 23. Experimental observation of the consequences of $e / \pi \neq 1$. Results of measurements of pion absorption in undercompensating, compensating, and overcompensating calorimeters. In diagram (a), the energy resolution $\sigma / E \cdot \sqrt{E}$ is given as a function of the pion energy, showing deviations from scaling for noncompensating devices. In diagram (b), the signal per $\mathrm{GeV}$ is plotted as a function of the pion energy, showing signal nonlinearity for noncompensating detectors (Fabjan and Wigmans, 1989).
It is instructive to analyze $n / \mathrm{mip}$, because of the very large number of neutrons with $E_{n} \lesssim 20 \mathrm{MeV}$ and the richness and intricacies of $n$-induced nuclear reactions. A variety of processes such as $\left(n, n^{\prime}\right),(n, 2 n),(n, 3 n)$, ( $n$, fission) in addition to elastic scattering take place in high- $Z$ materials. The ultimate fate of neutrons with energies $E_{n} \leqslant 1-2 \mathrm{MeV}$ is dominated by elastic scattering; cross sections are large $(\sim$ barns $)$ and mean free paths short (a few centimeters); the energy loss is $\sim 1 / A$ (target) and hence small. Once thermalized, a neutron will be captured, giving rise to $\gamma$ emission.

This abundance of neutrons gives a privileged role to hydrogen, which may be present in the readout material.
Because of the large $n$ - $p$ elastic cross section, on average half of the neutron kinetic energy is transferred. The recoil proton produced in the active material contributes directly to the calorimeter signal, i.e., is not sampled like a mip (a $1-\mathrm{MeV}$ proton has a range of $\sim 20 \mu \mathrm{m}$ in the scintillator). The second important $n$ reaction is the production of excitation photons through the $\left(n, n^{\prime}, \gamma\right)$ reaction (Wigmans, 1988).

This difference in response between high- $Z$ absorbers and hydrogen-containing readout materials has a consequence. Consider the contributions of $n / \mathrm{mip}$ as a function of $f_{\text {samp }}$. The mip signal will be decreased proportionally by increasing the thickness of the absorber 

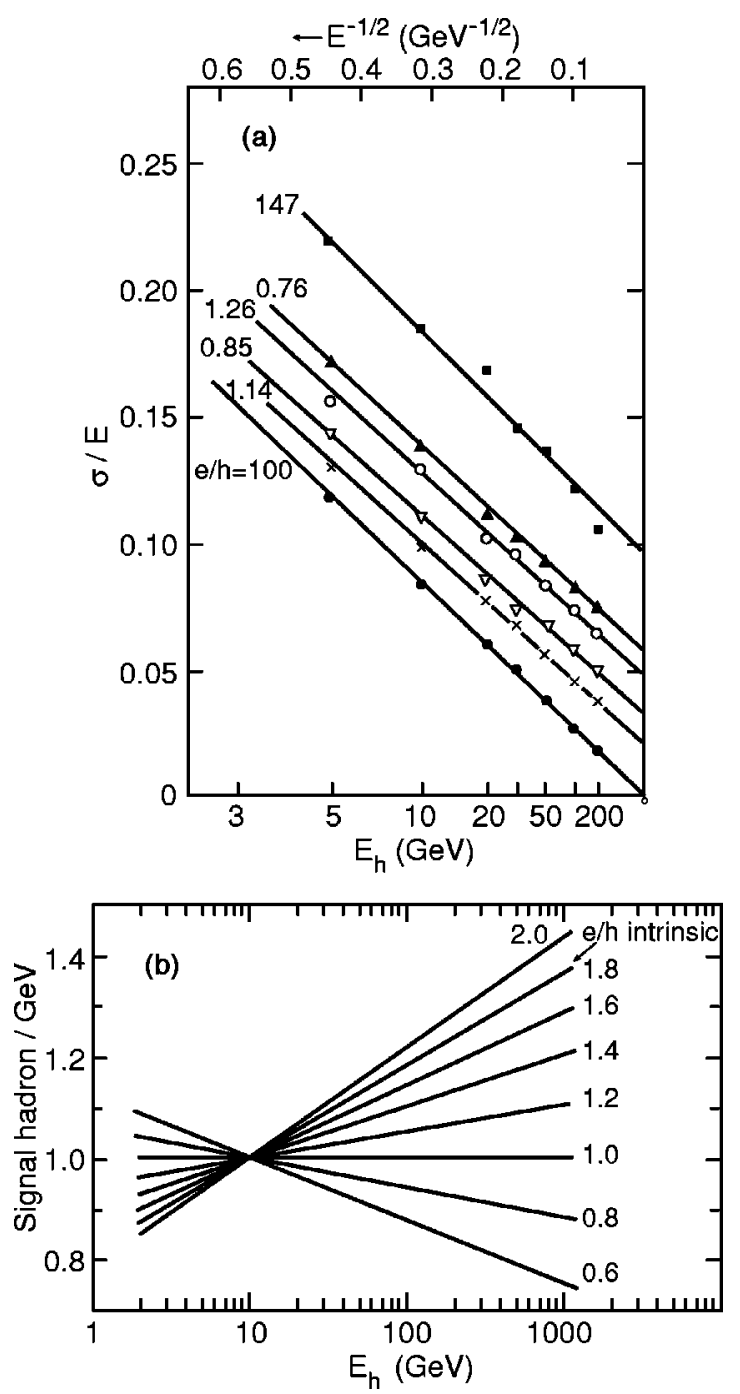

FIG. 24. Monte Carlo simulation of the effects of $e / \pi \neq 1$ on energy resolution (a) and response linearity (b) of hadron calorimeters with various values for $e / h$ (intrinsic), where $h$ (intrinsic) denotes the response to the purely hadronic component of the shower (Wigmans, 1988). plates, i.e., decreasing $f_{\text {samp }}$, whereas the signal from proton recoils will not be affected. As a consequence, the $n /$ mip signal will contribute more significantly to $e / \pi$. Therefore changing the sampling fraction allows $e / \pi$ to be altered. An example is shown in Fig. 25. The tuning of the ratio $R_{d}=$ passive material [mm]/active material $[\mathrm{mm}]$ is a powerful tool for acting on $e / \pi$ (Wigmans, 1988).

How tightly are the various contributions to the invisible energy correlated with the average behavior as measured by $e / \pi$ ? A quantitative answer needs rather complete shower and signal simulations. Two examples are shown in Fig. 26. One observes a significant reduction in the fluctuations and an intrinsic hadronic energy resolution of $\sigma / E \sim 0.2 \sqrt{E(\mathrm{GeV})}$. In principle, tuning of $e / \pi$ can be applied to all sampling calorimeters, opening the way to better performance.

There are several further consequences if $e / \pi \neq 1$. The energy resolution, which no longer scales with $1 / \sqrt{E}$, is usually approximated by $\sigma / E=a_{1} / \sqrt{E} \oplus a_{2}$, where a "constant" term $a_{2}$ is added quadratically, even though physics arguments suggest $a_{2}=a_{2}(E)$ (Wigmans, 2000). Furthermore, the hadronic fraction $F_{h}$ becomes different for pions $\left[F_{h}(\pi)\right]$ and protons or neutrons $\left[F_{h}(p)\right]$, typically $F_{h}(\pi) \simeq 0.85 F_{h}(p)$, resulting in differences of response in calorimeters with $e / \pi \neq 1$ (Gabriel et al., 1994; Akchurin et al., 1998).

This analysis implies another, at first sight, surprising result. A homogeneous hadron calorimeter (some purists may consider a BGO crystal hadron calorimeter) will have an $e / \pi \sim 1.4$ and therefore will be a rather lousy hadron calorimeter, which cannot compete with a run-of-the-mill but carefully designed $e / \pi=1$ leadscintillator calorimeter. Only a homogeneous $Z=1$ (liquid-hydrogen) calorimeter would have $e / \pi=1$ and would match the performance of a sampling calorimeter.

To complete the analysis of the contributions to the energy resolution we need to consider sampling fluctuations. For electromagnetic calorimeters we gave a simple explanation and an empirical parametrization:
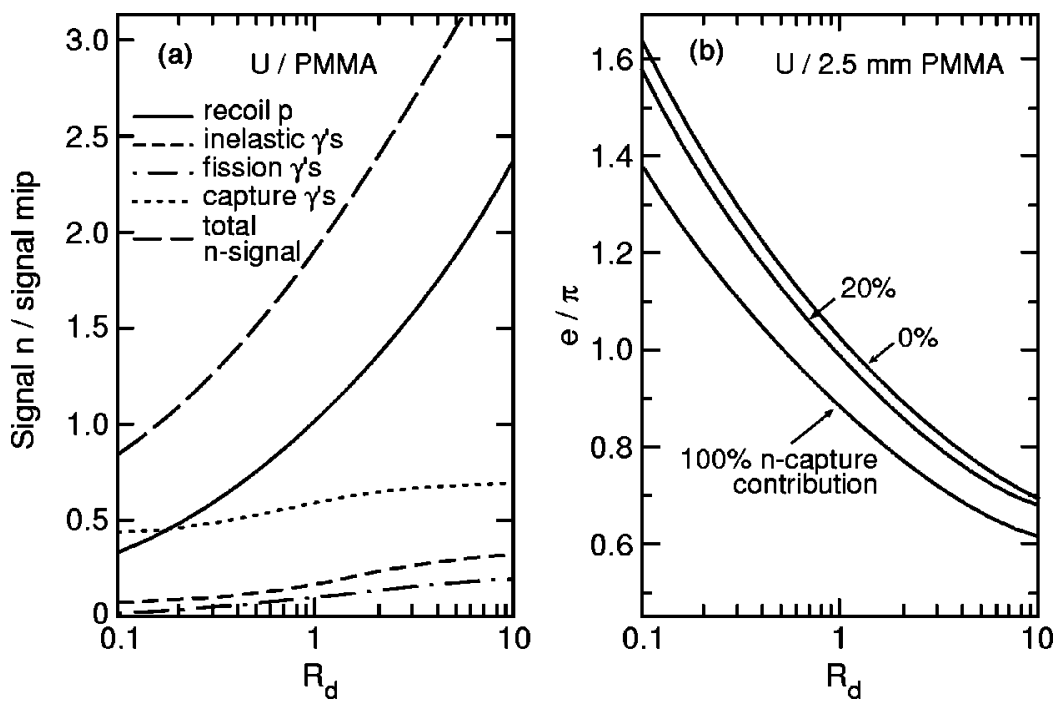

FIG. 25. (a) Different contributions to the signal ratio $\mathrm{n} / \mathrm{mip}$, for $\mathrm{U}$-plastic scintillator calorimeters, as a function of the ratio of the thicknesses of passive and active layers $R_{d}$. (b) The $e / \pi$ ratio as a function of $R_{d}$, assuming that 0,20 , or $100 \%$ of the $\gamma$ released in thermal neutron capture contribute to the calorimeter signal (Wigmans, 1988). 


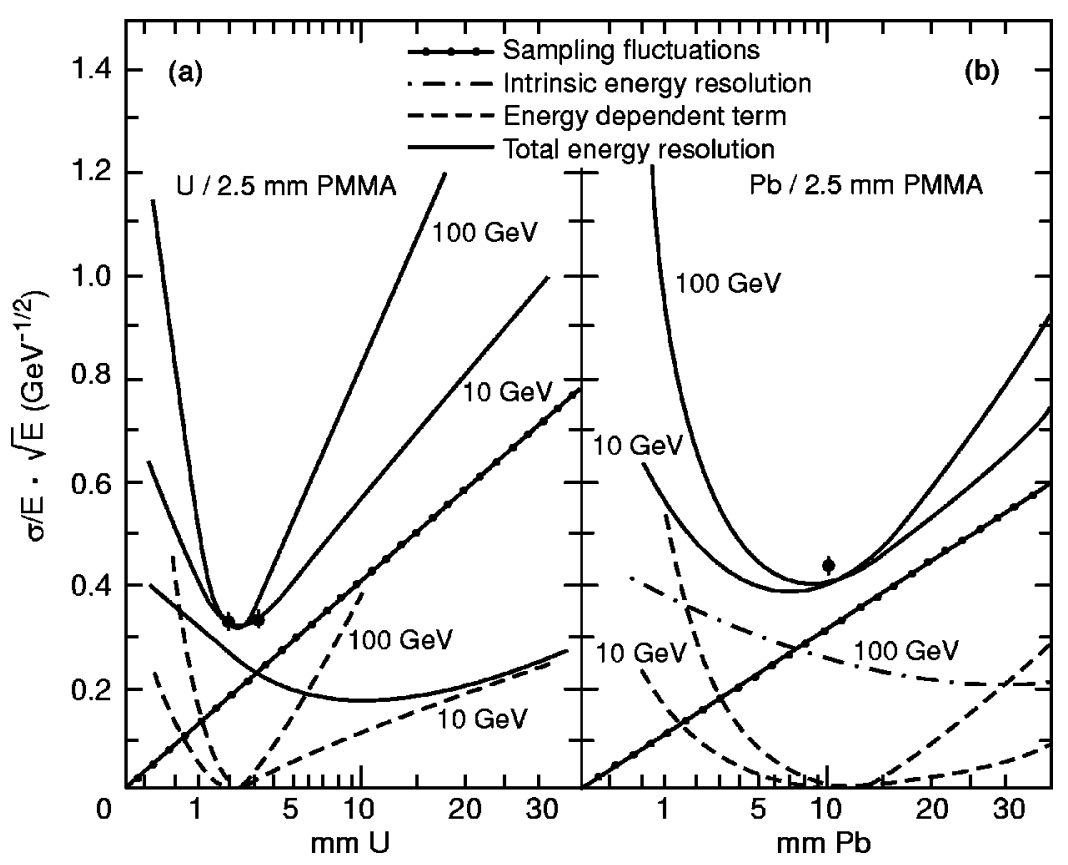

FIG. 26. The total energy resolution and the various contributions for the detection of 10and $100-\mathrm{GeV}$ hadrons in scintillator calorimeters, as a function of the thickness of the uranium plates (a) and of the lead plates (b). In both cases the thickness of the scintillator plates is $2.5 \mathrm{~mm}$. The dots in the curves are measured resolution values of actual calorimeters (Wigmans, 1988).

$$
\sigma_{\mathrm{samp}} / E=c \cdot[\Delta E(\mathrm{MeV}) / E(\mathrm{GeV})]^{1 / 2},
$$

where $\Delta E$ is the energy lost in one sampling cell and $c(\mathrm{em}) \simeq 0.05$ to 0.06 for typical absorber and readout combinations.

Similar arguments apply for the hadronic cascade; empirically, it has been observed that $c(\pi) \simeq 0.09$ in this case (Fabjan, 1987; Drews et al., 1990). For highperformance hadron calorimetry, sampling fluctuations cannot be neglected.

We can summarize the foundations of modern, optimized hadron calorimetry as follows:

- the key performance parameter is $e / \pi=1$, which guarantees linearity, $E^{-1 / 2}$ scaling of the energy resolution, and best resolution;

- by proper choice of type and thickness of active and passive materials the response can be tuned to obtain $e / \pi \simeq 1$;

- the intrinsic resolution in practical hadron calorimeters can be as good as $(\sigma / E) \cdot \sqrt{E} \leqq 0.2$;

- sampling fluctuations contribute at the level of

$$
\sigma / E \approx 0.09[\Delta E(\mathrm{MeV}) / E(\mathrm{GeV})]^{1 / 2} \text {. }
$$

\section{Monte Carlo codes for hadronic cascade simulation}

Modern calorimetry would not have been possible without extensive shower simulation. The first significant use of such a technique was aimed at the understanding of electromagnetic calorimeters. For example, electromagnetic codes were used in the optimization of $\mathrm{NaI}$ detectors in the pioneering work of Hofstädter, Hughes, and collaborators (Hughes, 1972). Over the years one code, EGS, has become de facto the world standard for electromagnetic shower simulation (Nelson, Hirayama, and Rogers, 1985).

Early hadronic cascade simulations were motivated by experimental work in cosmic-ray physics (Murzin, 1967) and sampling calorimetry (Ranft, 1970). However, it was the codes developed by the Oak Ridge group (Gabriel and Amburger, 1974), with their extensive modeling of nuclear physics, neutron transport, spallation, and fission, which became indissociable from the development of modern hadron calorimetry (Fabjan and Willis, 1975).

Today, for applications at accelerators and cosmic-ray studies, reliable codes are needed, modeling the physics from thermal neutrons $(\mathrm{meV})$ to hadrons of up to the $10^{20}$-eV scale.

We shall give a general description of the myriad approaches used (Wellisch, 1999) and highlight the present status by comparing experimental measurements with models in certain taxing cases. A recent overview can be found in Kling (2001).

The principal requirements for modern shower simulation codes are as follows:

- to be tuneable to reproduce the available experimental data;

- to provide for the possibility of extrapolating beyond accessible accelerator energies;

- to include the nuclear physics needed to describe low-energy neutron and photon production and interactions;

— to allow for the possibility of "event biasing," i.e., to artificially enhance certain reactions in order to economize CPU time;

— to include "customized code" for specialized simulation tasks.

\section{Shower physics modeling techniques}

For practical purposes three approaches are used to describe hadronic interactions, each with a distinct range of applications.

(a) The data-driven models incorporate experimental information in the modeling. They are particularly 
relevant in the domains of low-energy neutron scattering, photon evaporation, evaluation of inclusive cross sections, and isotope production. The codes [examples are MARS (Azhgirey et al., 1996), MORSE (Emmet, 1975), GEANT4 (GEANT4 Collaboration, 1994)] rely on neutron data libraries such as JENDL (Nakagawa et al., 1994) or FENDL (Wienke and Herman, 1998). Furthermore, these codes [e.g., GEANT4, HERMES (Cloth et al., 1988)] are used to simulate photon evaporation at low to moderate excitation energies, using, for example, the ENSDF data library (Bhat, 1992). Typically, one of these codes is used in any of the global simulations to model neutron interactions below $\sim 20 \mathrm{MeV}$.

(b) The parametrization-driven models aim to parametrize and extrapolate cross sections that are used over the full range of hadronic shower energies. In their modeling power they are situated between the "fast" parametrized models and the complex hadronic-interaction codes. They reproduce well inclusive data and global shower properties. They usually do not contain internal correlations and energy conservation. Well-known examples are GEISHA (Fesefeldt, 1985) and to a certain extent GCALOR (or GEANT-CALOR) (Zeitnitz and Gabriel, 1996).

(c) The hadronic-interaction models are the basis of or part of many currently used Monte Carlo programs. Besides modeling high-energy phenomena, they represent the soundest way of extrapolating beyond presently available test beam energies. Various forms of string models are used (Pi, 1992; Ranft, 1997) at high energy (above several GeV). The FLUKA code (Ferrari and Sala, 2001) uses the string model approach for the high-energy domain, hadronic-interaction modeling from $\sim 20 \mathrm{MeV}$ to a few $\mathrm{GeV}$, and data-driven modeling in the hadronic energy regime below $20 \mathrm{MeV}$.

\section{Applications: Illustrative examples}

We present comparisons of simulation with experiment to illustrate the quality of shower modeling.

Figure 27 presents modeling by three different codes of the $\pi^{0}$ component versus energy, and compares the results of this modeling with a measurement in the ATLAS Tile calorimeter (Kulchitsky, 2000). The different models vary in their prediction of this very fundamental quantity by up to $\sim 15 \%$. A parametrization of this component (Groom, 1998) is also shown. In Fig. 28 measurements of the energy resolution of the ATLAS $\mathrm{Cu}-\mathrm{LAr}$ calorimeter are compared with three different simulations, showing a rather wide spread for a relatively basic quantity (Schacht, 2000). One reason for this disappointing result may be the incomplete modeling of the readout response, so crucial for the understanding of hadronic calorimeters. In contrast, rather good agreement is obtained for a more complex calorimeter using the FLUKA code (Ferrari and Sala, 2001).

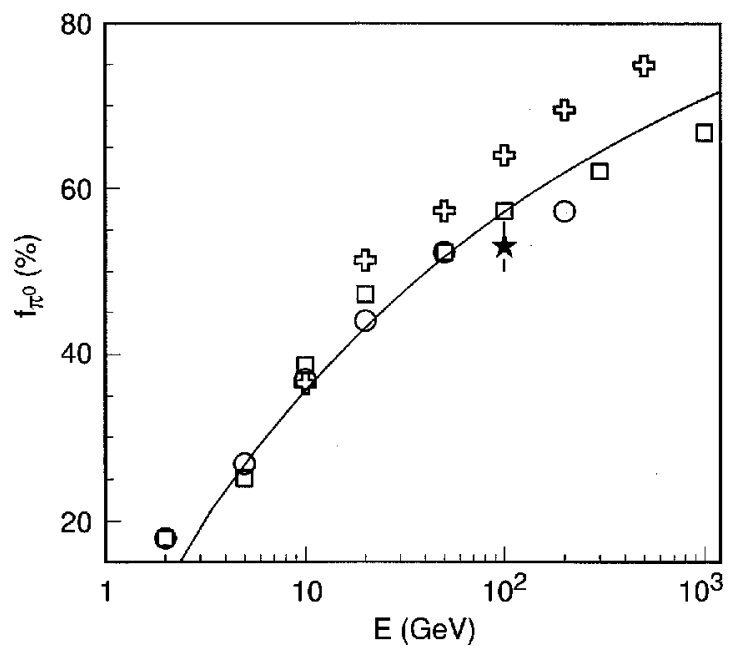

FIG. 27. The fraction of $\pi^{0}$ 's, $f\left(\pi^{0}\right)$, produced in hadronic showers versus energy. The star is a measurement, squares are GCALOR, circles GEISHA, and crosses CALOR predictions. One parametrization is also shown. From Kulchitsky (2000).

The quality of the low-energy neutron simulations can be gauged from Fig. 29. It shows the radially integrated production of ${ }^{115 \mathrm{~m}} \mathrm{In}$ from ${ }^{115} \mathrm{In}$ dosimeters for different energies and absorbers. These dosimeters are sensitive to neutrons in the $0.8-15-\mathrm{MeV}$ range through the reaction ${ }^{115} \operatorname{In}\left(n, n^{\prime}\right){ }^{115 \mathrm{~m}} \mathrm{In}$. The measurements are compared with the FLUKA calculations. The agreement is remarkable, better than 20\% (Fasso et al., 1983).

A particularly challenging application of these Monte Carlo techniques is in the range beyond present accelerator energies. As discussed in Sec. VI, the use of the

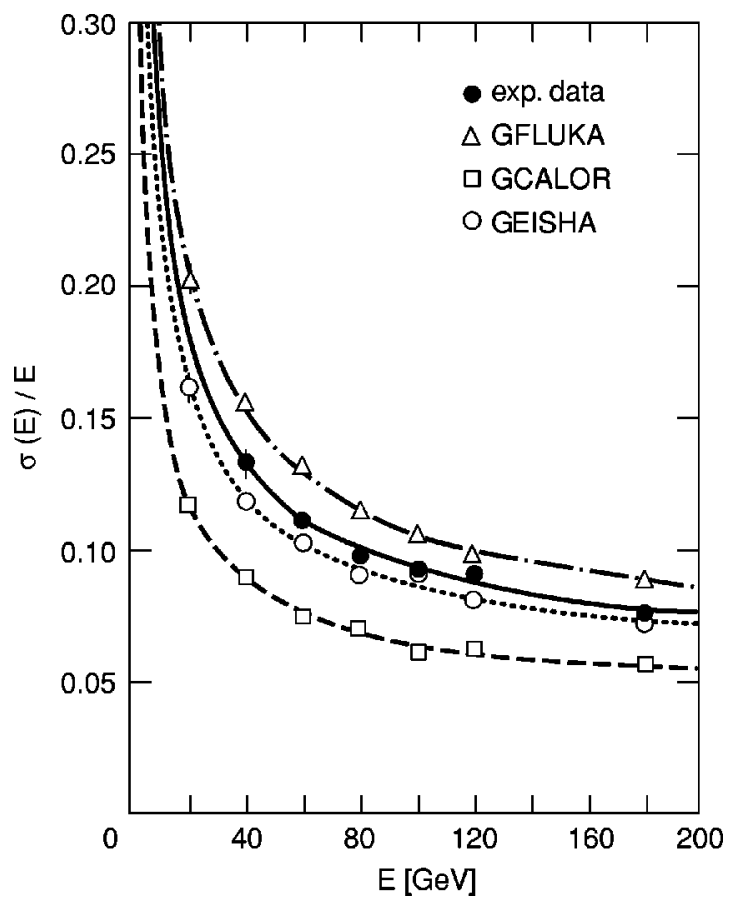

FIG. 28. Preliminary energy resolution data of an ATLAS LAr-Cu hadronic calorimeter module and comparison with three different Monte Carlo codes (Schacht, 2000). 


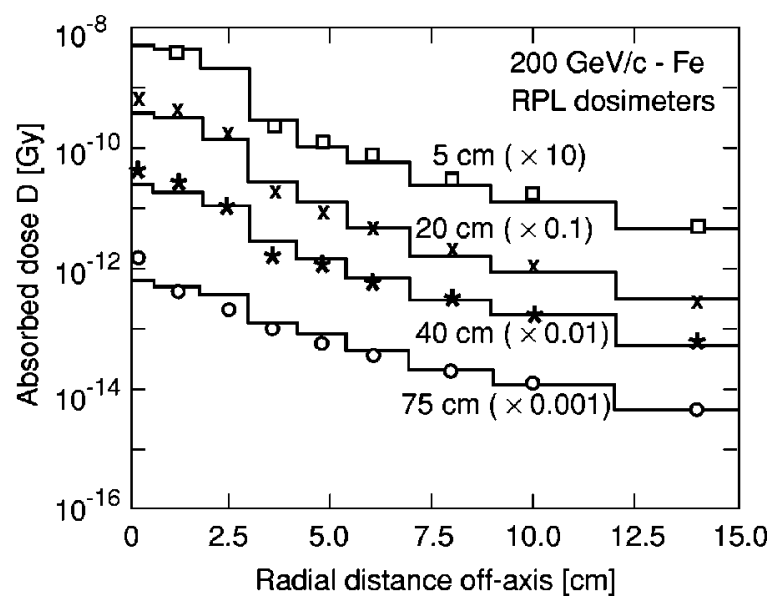

FIG. 29. Comparison of the calculated and measured production of ${ }^{115 \mathrm{~m}} \mathrm{In}$ from ${ }^{115} \mathrm{In}$ (radially integrated) as a function of depth in $\mathrm{Fe}$ and $\mathrm{Pb}$. These In dosimeters are sensitive to neutrons in the 0.8-15-MeV range (Fasso et al., 1983).

Earth's atmosphere as a hadronic detector allows cosmic hadrons and nuclei up to and beyond $10^{20} \mathrm{eV}$ to be probed. This requires "dead reckoning" of the detector response based on Monte Carlo techniques. In particular, considerable extrapolations of and faith in the simulation models are needed to establish the absolute energy scale. The estimate of the primary energy is based on knowing the shower shape and length: knowledge of $f_{\pi^{0}}$, the nucleon-nucleon cross section, particle multiplicities, transverse momentum distributions, etc., all contribute. Recently, various groups have agreed on the need to check their generators more critically and to assess the systematics of the energy measurements. As a caveat, Fig. 30 shows the range of predictions at relatively low energy $\left(10^{15} \mathrm{eV}\right)$ given by several frequently used Monte Carlo suites (Heck et al., 1999; Kampert et al., 2001; Knapp et al., 2003). The prediction for muons and electrons, typically the basis for the energy estimate, varies by almost a factor 2 . For a comparative

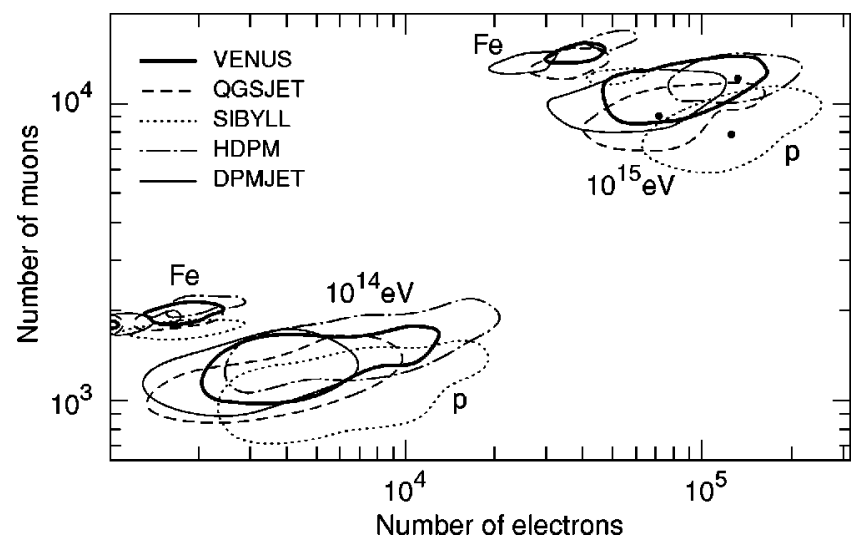

FIG. 30. Contours in the muon-number-electron-number plane for proton and iron showers in the Earth's atmosphere. Although the center-of-mass energies involved are close to those of present colliders, the results obtained with different codes show large differences (Heck et al., 1999).

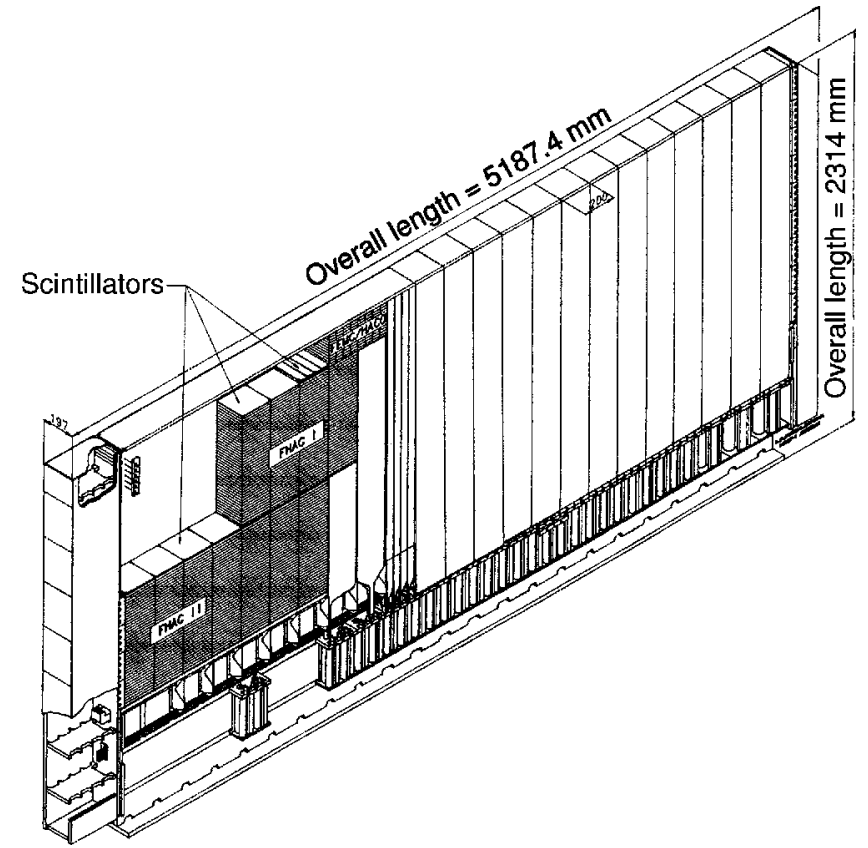

FIG. 31. View of one module of the ZEUS U-scintillator calorimeter. Wavelength-shifter readout is used to read cells of 5 $\times 20-\mathrm{cm}^{2}$ cross section in the electromagnetic compartment and of $20 \times 20 \mathrm{~cm}^{2}$ in two subsequent hadronic compartments (Derrick et al., 1991).

discussion of different models at the ultrahigh energies, see Ranft (2001).

We conclude that

- modern calorimetry owes much to Monte Carlo modeling;

- nevertheless, predictions have to be taken cum granu salis, in particular the extrapolation into performance and energy regimes not accessible to experimental benchmarking. Caveat emptor.

\section{Examples of hadron calorimeter facilities}

A number of hadron calorimeters have been developed during the past 20 years. We briefly describe three of them, each one reflecting in its own way the state of the art.

Research at the $e p$ collider HERA requires first and foremost precision jet spectroscopy to study the underlying dynamics of $e$-quark collisions. As typical jet energies are of $\mathcal{O}$ (hundred $\mathrm{GeV}$ ), energy and position resolution for jets are at a premium. The H1 Collaboration developed a calorimeter based on the LAr-Pb and LAr-Fe sampling technology. In this device a certain level of "off-line" compensation is achieved because hadron showers are longitudinally measured up to ten times and longitudinal shower weighting can be applied (Andrieu et al., 1993).

The ZEUS Collaboration at HERA (Derrick et al., 1991) developed an intrinsically compensated calorimeter using the U-scintillator sampling technique, modeled after the pioneering Axial Field Spectrometer facility (Akesson et al., 1985). The ZEUS calorimeter is 


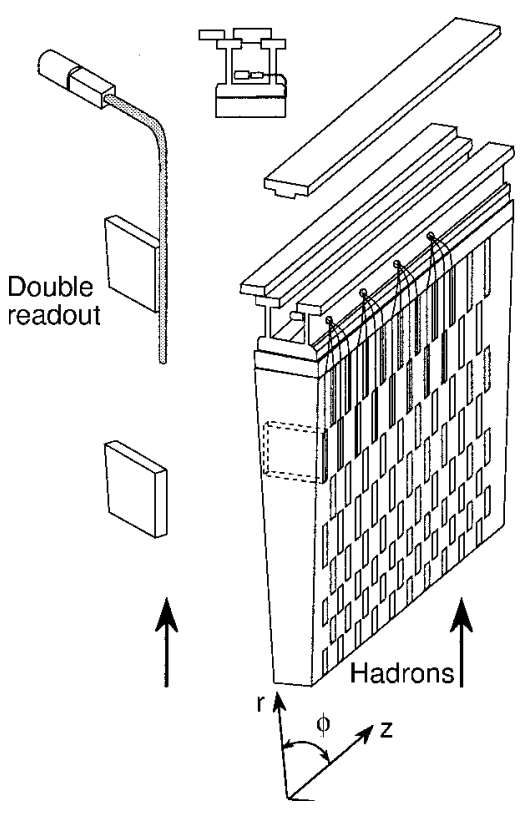

FIG. 32. View of one module of the ATLAS hadronic barrel calorimeter. Thirty-six such modules complete the cylindrical detector. Each of the longitudinally oriented scintillating tiles is read with two wavelength-shifting fibers (ATLAS Collaboration, 1996c).

constructed in a modular form (Fig. 31), with units measuring approximately $5 \mathrm{~m}$ long by $20 \mathrm{~cm}$ wide with a depth of more than $2 \mathrm{~m}$. The ratio of the thickness of the ${ }^{238} \mathrm{U}$ plates $(3.3 \mathrm{~mm})$ to the scintillator plates $(2.6 \mathrm{~mm})$ was tuned to achieve $e / \pi=1$, confirmed by measurements to be $e / \pi=1.00 \pm 0.03$. The measured hadronic energy resolution, $\sigma / E$ (hadrons $)=0.35 / \sqrt{E(\mathrm{GeV})}$, is consistent with a sampling resolution of $\sigma / E($ sampling, hadrons $)=0.23 / \sqrt{E(\mathrm{GeV})}$ and an intrinsic resolution of $\sigma / E$ (intrinsic, hadrons) $=0.26 / \sqrt{E(\mathrm{GeV})}$. The price to pay for optimized hadronic performance is a rather coarse sampling frequency, which reflects in the electron energy resolution $\sigma / E($ electrons $)=0.18 / \sqrt{E(\mathrm{GeV})}$.

A novel calorimeter facility, presently under construction for the ATLAS experiment at the LHC (ATLAS Collaboration, 1996a), has been optimized for a different physics program (see Sec. IV.A).

A "hybrid" solution has been chosen: the electromagnetic calorimeter uses the accordion geometry (see Sec. II.C.2) and represents approximately one interaction length $\lambda$. The subsequent eight $\lambda$ are based on a scintillator tile-Fe plate structure, albeit in a novel and unconventional geometry (Fig. 32). For ease and economy of construction, the tiles are mostly oriented along the shower axis; this causes no degradation of performance. This geometry also greatly facilitates longitudinal and transverse segmentation and permits effective longitudinal weighting of the shower energy. Weighting leads to an energy resolution of $\sigma / E \approx(0.42 / \sqrt{E}+0.018 / E)$ $\oplus 1.8 / E$ and a good linear response (Akhmadaliev et al., 2002).

\section{CALORIMETER OPERATION IN ACCELERATOR EXPERIMENTS}

In this section we discuss the operation of calorimeters in accelerator experiments. First, examples of performance requirements are listed, then integration issues are described, and finally calibration techniques and strategies presented.

\section{A. Performance requirements}

The operation of calorimeters in modern experiments sets a large number of stringent requirements, both from the technical (e.g., fast response, low noise, dynamic range) and physics performance (e.g., good energy, space, and time resolution, particle identification) points of view. Constraints come also from the integration with the rest of the experiment, for example, the presence of a magnetic field, and from environmental conditions such as radiation levels. Therefore the choice of the optimal detector technique and geometry is a multidimensional problem which takes into account all the above issues as well as cost.

The requirements, and therefore the calorimeter choice, are obviously different for different applications. Since operation at the LHC imposes particularly severe demands in terms of physics and technical performance, the main requirements of the ATLAS and CMS calorimeters (ATLAS Collaboration, 1996a; CMS Collaboration, 1997) are discussed below as examples.

- Fast response. At the LHC design luminosity of $10^{34} \mathrm{~cm}^{-2} \mathrm{~s}^{-1}$, on average 25 events are expected to be produced at each bunch crossing, i.e., every $25 \mathrm{~ns}$. These are mostly soft interactions (called minimumbias events), characterized by low- $p_{T}$ particles in the final state. When, occasionally, an interesting high- $p_{T}$ physics event occurs, for instance, the production of a $W$ boson or a Higgs boson, this event will be overlapped with (on average) 25 minimum-bias events produced in the same bunch crossing. If the detector response is not fast enough, signals from other soft interactions from the preceding and following bunch crossings will also be present. This overlap of events is called "pileup." At each bunch crossing, the pileup of $\sim 25$ minimum-bias events produces about 1500 particles in the region $|\eta|<2.5$ with average transverse momentum $\sim 500 \mathrm{MeV}$, giving a total average transverse energy of $\sim 1 \mathrm{TeV}$. Although this average transverse energy can be subtracted, the event-by-event fluctuations cannot and produce a smearing of the calorimeter response (called pileup noise) which contributes in the form $\sim$ (pileup r.m.s.) $/ E$ to the energy resolution. To reduce the magnitude of this contribution, i.e., the pileup r.m.s., a fast calorimeter response (at the level of $50 \mathrm{~ns}$ or faster) is needed in order to integrate over a minimum number of bunch crossings. This in turn requires high-performance readout electronics. With a response time of $\sim 50 \mathrm{~ns}$, the typical pileup r.m.s. over a region containing an electromag- 
netic shower is $E \sim 250 \mathrm{MeV}$ in the central part of the detector (at $\sim 90^{\circ}$ from the beam axis), which gives a contribution of $2.5 \%$ to the energy resolution of $E$ $=10 \mathrm{GeV}$ electrons. In addition, a fine calorimeter granularity is also important in order to minimize the probability that pileup particles hit the same cell as an interesting object (e.g., an electron from a possible $H \rightarrow 4 e$ decay).

- Radiation hardness. Because of the huge flux of particles produced by the high-rate $p p$ collisions, the LHC experimental environment will be characterized by high levels of radiation. Over ten years of operation, the calorimeter regions at $|\eta| \sim 5$, where the radiation is the greatest because of the high energy density of the particles hitting the forward parts of the detector, will be exposed to a flux of up to $\sim 10^{17}$ neutrons $/ \mathrm{cm}^{2}$ and to a dose of up to $10^{7} \mathrm{~Gy}$ ( $1 \mathrm{~Gy}=1 \mathrm{~J} \mathrm{~kg}^{-1}$ is a unit of absorbed energy). Radiation-hard detectors and electronics are therefore needed, as well as quality control and radiation tests of every single piece of material installed in the experiment.

- Angular coverage. LHC calorimeters must be hermetic and cover the full azimuthal angle and the rapidity region $|\eta|<5$ (i.e., down to $1^{\circ}$ from the beam axis). This is required mainly for a reliable measurement of the event total transverse energy, which is in turn needed to detect neutrinos (or other hypothetical weakly interacting particles). These particles can only be detected indirectly by observing a significant amount of missing transverse energy in the final state.

Since energy losses in poorly instrumented regions of the apparatus or along the beam axis could fake a neutrino signal, the calorimeters have to cover as much of the solid angle as possible. For example, for events containing a supersymmetric Higgs with $m_{H}$ $=150 \mathrm{GeV}$ decaying into $\tau$ pairs and for an ideal calorimeter with infinite resolution, the missing transverse energy would be measured with an r.m.s. of $\sim 2 \mathrm{GeV}$ if the calorimeter coverage extended over $|\eta|<5$, and $\sim 8 \mathrm{GeV}$ if the coverage extended over $|\eta|<3$. This deterioration is due to particles escaping detection because produced at small angles from the beam line.

- Excellent electromagnetic energy resolution. This is needed, for instance, to extract a possible $H \rightarrow \gamma \gamma$ signal from the irreducible background of $\gamma \gamma$ events produced by known processes. Since the irreducible background is typically a factor of $\sim 50$ larger than the signal for a Higgs mass below $\sim 150 \mathrm{GeV}$, a very good mass resolution, at the level of $1 \%$, is needed in order to observe a narrow resonance above the $\gamma \gamma$ irreducible background.

- Angle measurements. The energy resolution is not the only contribution to the width of the reconstructed $\gamma \gamma$ invariant mass distribution from $H \rightarrow \gamma \gamma$ decays. In order to reconstruct the two-photon invariant mass it is necessary to know the direction of both photons.
Typically, an angular resolution of $\sigma_{\theta} \sim 50 \mathrm{mrad} /$ $\sqrt{E(\mathrm{GeV})}$ is required to achieve a $\gamma \gamma$ mass resolution of $1 \%$.

- Large dynamic range. Electrons in particular need to be measured with accuracy over an unprecedented energy range going from a few $\mathrm{GeV}$ up to $\sim 3 \mathrm{TeV}$. This large range is required on the one hand to detect the soft electrons produced in the decays of $b$ hadrons, and, on the other hand, to look for heavy particles decaying into electrons (e.g., additional gauge bosons $W^{\prime}$ and $Z^{\prime}$ ) up to masses of $\sim 6 \mathrm{TeV}$.

The readout systems of the LHC electromagnetic calorimeters must be sensitive to signals as low as $\sim 50 \mathrm{MeV}$ (which is the typical electronic noise per channel) and as high as $3 \mathrm{TeV}$ (which is the maximum energy deposited in one calorimeter cell by electrons produced in the decays of $Z^{\prime}$ and $W^{\prime}$ with masses $\sim 6 \mathrm{TeV}$ ). This corresponds to a dynamic range of $10^{5}$, which is realized in practice by using multigain electronic chains. A smaller dynamic range would increase the electronic noise because of a significant contribution of the quantization noise.

- Jet energy resolution and linearity. The jet energy resolution needs to be at the level of $\sim 50 \% / \sqrt{E(\mathrm{GeV})} \oplus 3 \%$ for the LHC physics program (ATLAS Collaboration, 1999). This includes a precise measurement of the top mass; the search for the Higgs boson in the $t t H$ channel with $H \rightarrow b \bar{b}$, which demands a good $b \bar{b}$ mass resolution to observe a narrow peak over the large background; searches for new heavy resonances (e.g., a $Z^{\prime}$ ) decaying into two jets, etc. In particular, detection of new heavy resonances in the $\mathrm{TeV}$ mass range requires a small constant term since the latter is the dominant contribution to the energy resolution at high energy.

The linearity of the reconstructed jet energy should be better than $2 \%$ up to $\sim 4 \mathrm{TeV}$, which sets constraints on the quality of the calorimeter response to hadrons (implying a good level of compensation). This requirement comes from the fact that a calorimeter nonlinearity could produce an instrumental enhancement of the (steeply falling) QCD jet cross section at high energy, similar to the signature expected from quark compositeness. For instance, an uncorrected nonlinearity of $5 \%(2 \%)$ could fake a compositeness scale $\Lambda \sim 20 \mathrm{TeV}(\Lambda \sim 30 \mathrm{TeV})$.

- Particle identification. An efficient rejection of jets faking electrons and photons is needed for several physics studies at the LHC (e.g., Higgs searches). Usually a jet consists of many particles and can easily be distinguished from a single electron or photon because of the broader shower size in the calorimeters or the presence of several tracks. However, occasionally a jet can fake a single photon. This can happen if the original quark fragments into a very hard $\pi^{0}$ plus a few other very soft (and hence undetected) par- 
ticles, and the two photons from the $\pi^{0}$ decay are too close to be resolved. For a $\pi^{0}$ of $E \sim 50 \mathrm{GeV}$ the distance between the two decay photons is smaller than 1 $\mathrm{cm}$ at $150 \mathrm{~cm}$ from the interaction point (this is typically the distance at which electromagnetic calorimeters are located). Therefore the two photons appear as a single photon in the electromagnetic calorimeter, unless the latter has a fine enough granularity to be able to detect two distinct close-by showers. Although the probability for a jet to fragment into a single isolated $\pi^{0}$ is small, the cross section for di-jet production is, for instance, $\sim 10^{8}$ times larger than the $H$ $\rightarrow \gamma \gamma$ cross section, which makes it a dangerous background. This sets requirements on the granularity of the electromagnetic calorimeter, which must provide adequate $\gamma / \pi^{0}$ discrimination.

\section{B. Integration}

For most high-energy physics applications, calorimeters are integrated into complex experiments consisting of several subdetectors of which the calorimeter is only one component. This has both negative and positive consequences. The drawback is that calorimeters have to satisfy overall constraints, such as space limitations, and work in an environment that can deteriorate their response (material, magnetic field, etc.). In many cases additional devices or special software techniques are needed in order to recover, at least in part, the loss in performance. The advantage is that the calorimeter task can be made more effective by combining the calorimeter measurements with the information from other subdetectors. For instance, the use of an energy-flow algorithm, by which the momentum of charged particles is measured in the inner detector and the energy of neutral particles in the calorimeters, has allowed the ALEPH experiment to improve the energy resolution for $Z$ $\rightarrow q \bar{q}$ events by almost a factor of 2 compared to a purely calorimetric measurement of the event energy (Buskulic et al., 1995). Similar results have been obtained by the other LEP experiments.

Two issues related to the integration and performance of calorimeters in large-scale experiments are discussed here as examples: material effects and particle identification.

\section{Impact of material}

Energy losses in the material (e.g., from tracking devices) that particles have to traverse before reaching the active part of the calorimeter are most important for electrons and photons, and therefore most detrimental for the performance of electromagnetic calorimeters.

The material in the inner detectors of the LEP and Tevatron experiments is typically a few percent of a radiation length, but the inner detectors of future LHC experiments will be more massive. Furthermore, the coil providing the magnetic field in the inner cavity often sits in front of the electromagnetic calorimeter (for example, in the OPAL and ATLAS experiments), and the calo-

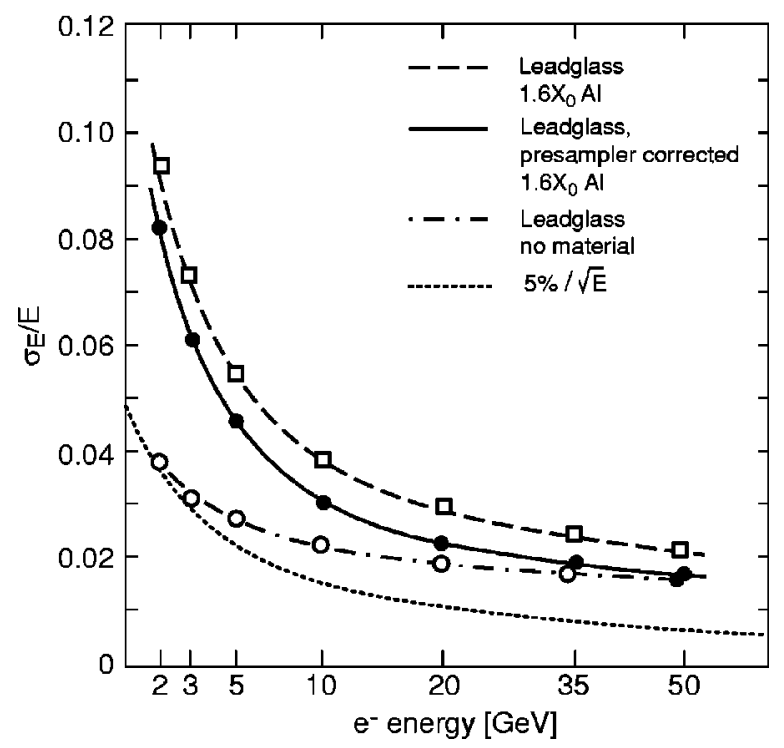

FIG. 33. Relative energy resolution of the OPAL lead-glass end-cap electromagnetic calorimeter as a function of the energy of the incident electron beam. The open circles show the calorimeter resolution with no material in front, the open squares show the calorimeter resolution with a $1.6 X_{0}$ - $\mathrm{Al}$ piece in front and no presampler corrections, the closed circles show the resolution obtained after presampler corrections (see text). From Beard et al. (1990).

rimeter support structures and cables provide additional dead layers (not to mention the contribution of the cryostat in the case of noble-liquid calorimeters).

Although the average energy lost by electrons and photons in the upstream material can be determined and corrected for, the event-by-event fluctuations cannot, unless dedicated devices are used (see below). These fluctuations provide an additional contribution to the energy resolution and, if the upstream material is large $\left(>1 X_{0}\right)$, can spoil the calorimeter intrinsic performance. This is illustrated in Fig. 33, which shows the electron energy resolution of the lead-glass end-cap electromagnetic calorimeter of the OPAL experiment as obtained in a beam test performed in various conditions (Beard et al., 1990). The open circles and open squares give the calorimeter energy resolutions without and with a slab of material ( $1.6 X_{0}$ of aluminum) in front, respectively. It can be seen that over the energy range 10-50 $\mathrm{GeV}$ the calorimeter resolution is deteriorated by a factor of 1.7-1.3 by the presence of additional material.

Both simulations and test beam measurements of various calorimeters have shown that an acceptable recovery of the energy resolution is possible by using dedicated devices, provided that the upstream material does not exceed 2.5-3 $X_{0}$. Examples of such devices are massless gaps and presampler detectors. These are thin layers of active medium placed in front of electromagnetic calorimeters or in the cracks between calorimeter parts (for instance, in the D0 and ATLAS calorimeters). Massless gaps are usually integrated in the calorimeter structure, whereas presamplers are separate devices read out independently. In both cases, the energy re- 
leased by the incident particles in these devices is proportional to the energy lost upstream. Therefore, by collecting the energy in these layers, and by adding it (suitably weighted) to the energy measured in the calorimeter, it is possible to recover for energy losses event by event, and thus take into account fluctuations. In most cases massless gaps and presamplers have a coarse granularity, since they are used for energy measurements and not for position measurements.

The OPAL end-cap presampler, which is made of thin multiwire gas chambers operated in saturated mode, is installed in front of the end-cap lead-glass calorimeter. As shown in Fig. 33, by adding the presampler energy, suitably weighted, to the lead-glass energy, about half of the deterioration in resolution due to the material upstream of the calorimeter is gained back at $10 \mathrm{GeV}$. At lower energies the improvement is smaller, at higher energies larger. If the material in front becomes too large $\left(2.5-3 X_{0}\right)$, then the correlation between the energy deposited upstream and the energy deposited in presamplers and massless gaps is lost. This is because lowenergy particles in the shower are completely absorbed by the dead material if this is too thick, and therefore they do not contribute to the signal in the active devices. This phenomenon is more pronounced the smaller the incident particle energy, which explains why at low energy the material effects are larger and recovery is more difficult (see Fig. 33).

\section{Particle identification}

Calorimeters with good transverse and longitudinal segmentation offer good particle identification capabilities. This is because hadronic showers are usually longer and broader than electromagnetic showers. Therefore by measuring the energy fractions deposited in the cells of a segmented calorimeter it is usually possible to distinguish incident hadrons from electrons and photons.

The particle identification capability can be made more powerful by combining the information from the calorimeter and other subdetectors. An example is presented in Fig. 34, which shows the expected electron/jet separation capability of the ATLAS experiment, as obtained from detailed GEANT simulations. The electromagnetic and hadronic calorimeters alone provide a jet rejection of 1000 , for more than $90 \%$ electron efficiency. Another factor of 10 in rejection is obtained by requiring a track in the inner detector to point to the shower in the calorimeter, and to match in momentum the shower energy. A rejection of more than $10^{5}$ is achieved by using in addition the Transition Radiation Tracker (ATLAS Collaboration, 1997) to distinguish charged hadrons from electrons, and by recognizing converted photons.

\section{Calorimeter calibration}

Calorimeter calibration has several purposes: to equalize the cell-to-cell output signals in order to obtain a response as uniform as possible and therefore a small

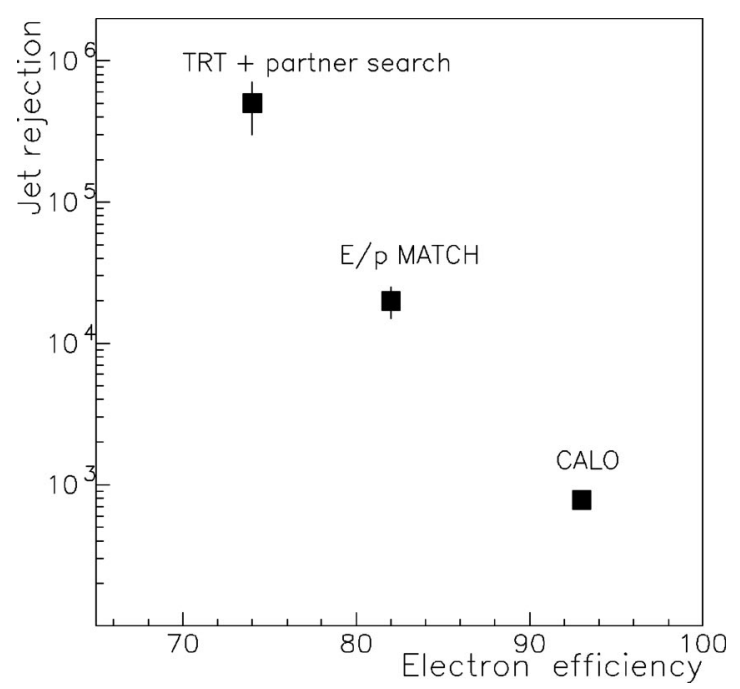

FIG. 34. Jet rejection as a function of the electron identification efficiency in the $p_{T}$ range $20-50 \mathrm{GeV}$, as obtained from a GEANT simulation of the ATLAS detector. The improvement obtained by using the information of the various subdetectors is shown (see text).

contribution to the constant term of the energy resolution; to set the absolute energy scale for electrons, photons, single hadrons, and jets; to monitor variations in the detector response with time. No single calibration system is able to achieve all these goals, therefore several methods are usually combined.

It should be noted that with the increasing energy of present and future machines, response uniformity, and therefore calibration, become more and more important issues. Furthermore, the increasing size and complexity of the experiments render the calibration and monitoring tasks very challenging, given that calorimeters are often equipped with a large number of channels (up to several hundred thousand).

Three main tools are usually employed to calibrate a calorimeter:

- Hardware calibration. This is mainly used to equalize and monitor the cell-to-cell response of the detector and of the associated electronics. The electronics calibration system injects a known pulse at the input of the readout chain. Channel-to-channel dispersions as small as $0.2 \%$ can be achieved. However, this system does not allow a calibration of the detector response, for which other devices (e.g., lasers, radioactive sources) are used that inject a well-known light or charge signal into the active elements of the detector. Their aim is to equalize the detector response, this time at the cell level, and to monitor variations of this response with time. In the ZEUS uranium-scintillator calorimeter a natural calibration source is provided by the radioactivity of the absorber.

- Test beam calibration. Usually some calorimeter modules are exposed to test beams before being installed in the final detector. One of the main aims of this step 

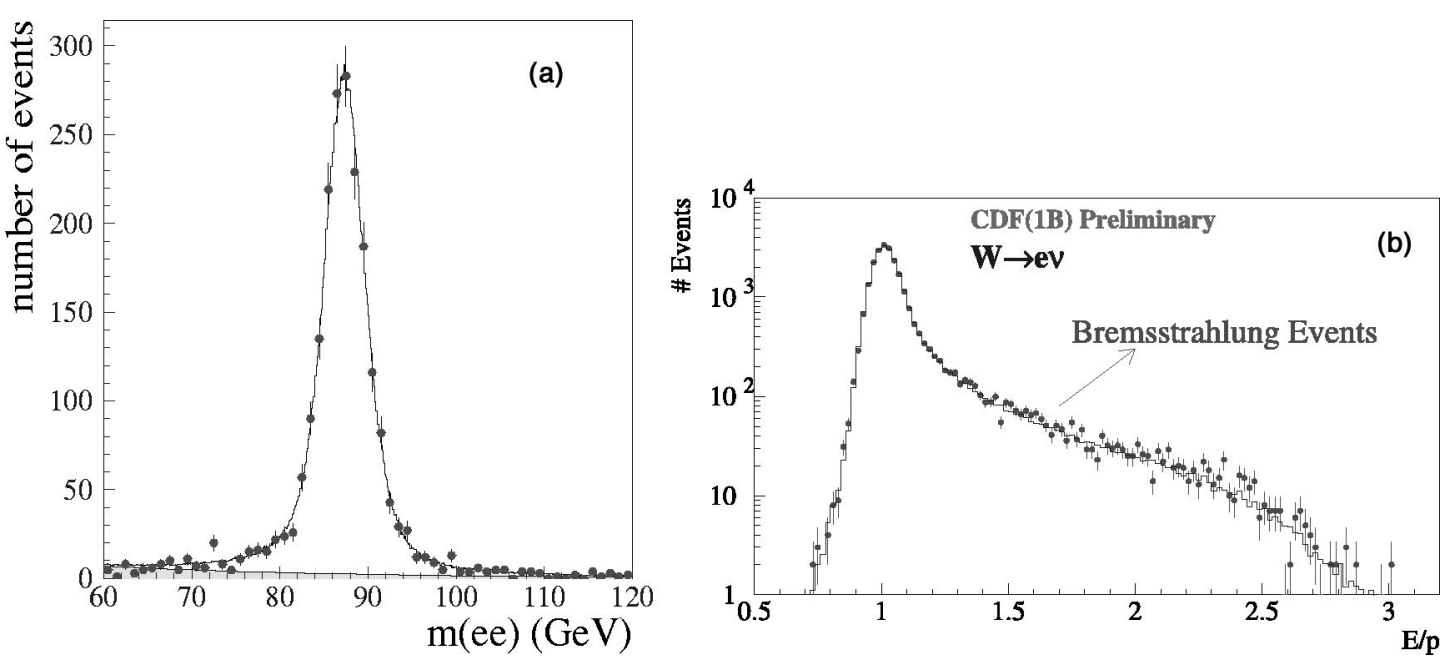

FIG. 35. (a) The di-electron mass spectrum reconstructed in the D0 central calorimeter before the final energy scale calibration for the run-I $Z \rightarrow e$ data sample (Abbott et al., 1998). The superimposed curve shows the fit. (b) The $E / p$ ratio for isolated electrons from $W$ decays as obtained from the CDF run-IB data (Abe et al., 1995; Kim, 1999). The superimposed curve shows the fit.

is to set a preliminary absolute energy scale for electrons and pions, given that the incident beam energy is well known.

- In situ calibration with physics samples. A further calibration step is needed after installation in the experiment. This is because the experimental environment, e.g., the presence of material in front of the calorimeter due to tracking devices, is not the same as at the test beam and is not seen by the hardware calibration. Furthermore, the calorimeter response to jets and the missing transverse energy cannot be measured at the test beam where only single particles are available. The in situ calibration allows one to correct residual nonuniformities, to understand the impact of the upstream material and of the environment, to follow the detector response variations with time, and to set the final absolute energy scale under experimental conditions. This is achieved by using well-known control physics samples, such as $Z \rightarrow e e$ events or $W \rightarrow j j$ decays in $t \bar{t}$ events.

One of the most challenging steps of the calorimeter calibration procedure is the setting of the absolute energy scale using physics samples. It is therefore briefly discussed here. Only the case of hadronic machines is considered, since at $e^{+} e^{-}$colliders the precise knowledge of the center-of-mass energy provides useful constraints and renders this operation easier.

The electromagnetic absolute energy scale at hadronic colliders is set mainly by using well-known resonances such as $\pi^{0} \rightarrow \gamma \gamma, J / \Psi \rightarrow e e, Y \rightarrow e e$ in the low-energy range and $Z \rightarrow e e$ at higher energies.

As an example, in the D0 experiment the calorimeter energy scale is calibrated by using $Z \rightarrow e e$ events and the relation $E_{\text {true }}=\alpha E_{\text {meas }}+\delta$, where $E_{\text {meas }}$ is the electron energy measured in the calorimeter and the parameters $\alpha$ and $\delta$ are varied until the reconstructed $Z$ mass peak agrees with the nominal value. Figure 35(a) shows the reconstructed $Z \rightarrow e e$ mass from the run-I data (Abbott et al., 1998) before the final scale calibration. The peak of the distribution is $\sim 5 \%$ below the nominal $Z$ mass. This wrong initial scale has been attributed mainly to the fact that no module of the final D0 central calorimeter was calibrated with test beams (only prototypes), and indicates the importance of performing such test beam measurements in order to keep the energy correction factors (and therefore the related systematic uncertainties) minimal.

An alternative (and complementary) method consists of transferring the energy scale from the tracker to the electromagnetic calorimeter by measuring the $E / p$ ratio for isolated electrons, where $E$ is the electron energy as measured in the calorimeter and $p$ is the electron momentum as measured in the inner tracker. This procedure involves several steps. The momentum scale in the inner tracker is first calibrated by using isolated muons, e.g., from $Z \rightarrow \mu \mu$ decays. The momentum scale for electrons is not automatically available at this stage because electrons lose part of their energy through bremsstrahlung. A Monte Carlo simulation of the tracker containing the details of the material distribution is used to compute the electron energy losses and hence obtain the initial electron momentum. Finally, the momentum scale is transferred to the electromagnetic calorimeter by adjusting the $E / p$ distribution for electrons to 1 . An example from CDF (Abe et al., 1995; Kim, 1999) is shown in Fig. 35(b).

By using these methods, a precision on the absolute electron energy scale of $\sim 0.1 \%$ has been achieved by both the CDF and D0 experiments. This precision is limited by the statistics of the above-mentioned physics samples. The dominant sources of systematic uncertainties are the incomplete knowledge of the tracker material, calorimeter response nonlinearities, and radiative $Z$ decays.

The uncertainty on the electron energy scale is the dominant systematic error on the $W$ mass as measured at the Tevatron in the electron-neutrino channel (Abe 


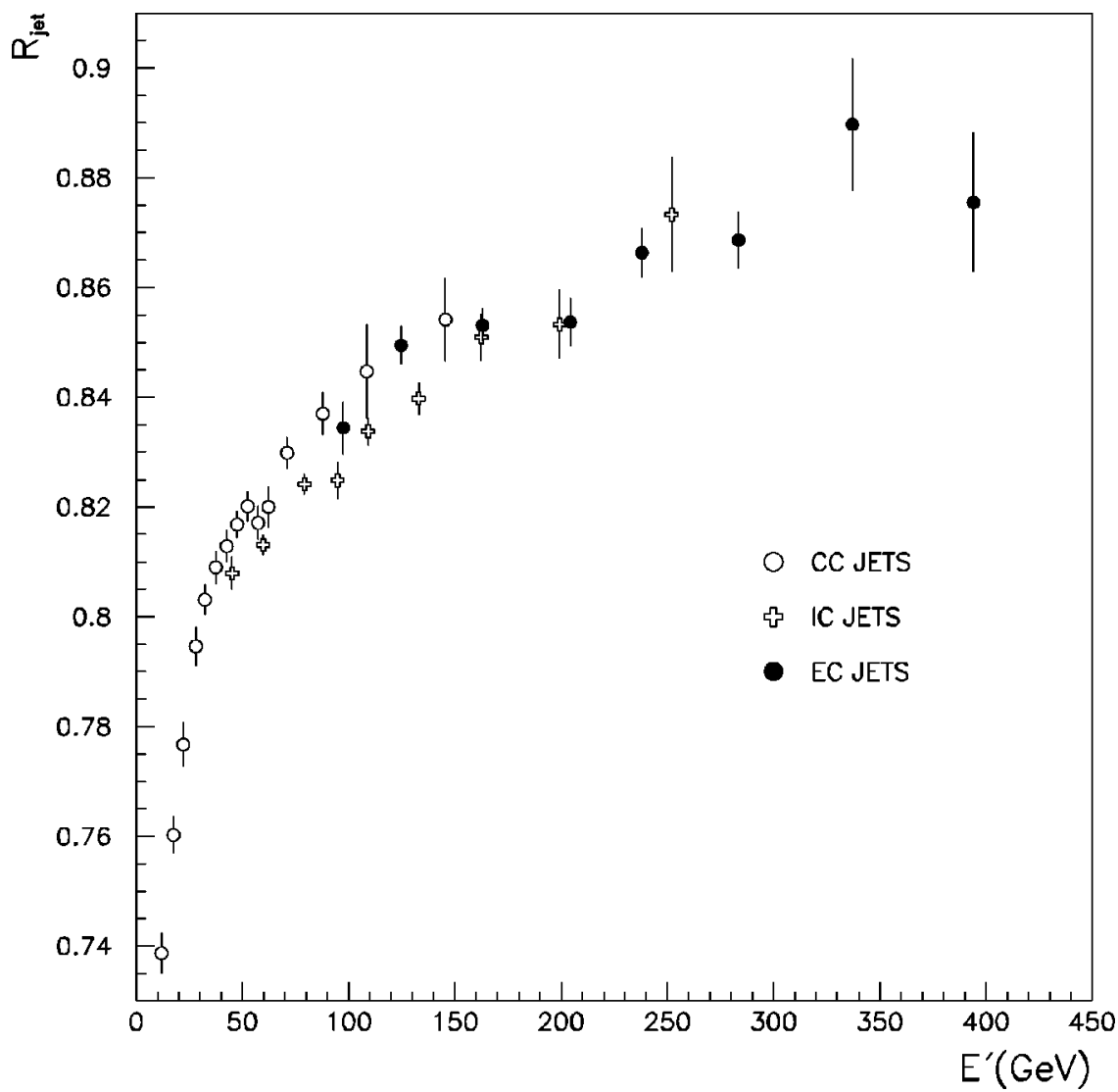

FIG. 36. Inverse of the correction factor to the jet energy as a function of energy, as obtained with a sample of $\gamma$-plus-one-jet events in the D0 experiment (run-I data). The different symbols indicate different calorimeter regions (central, end cap, intercryostat). From Abbott et al. (1999).

et al., 1995; Abbott et al., 1998; Kim, 1999). One of the LHC physics goals is to measure the $W$ mass to about 15 $\mathrm{MeV}$; this requires a calibration of the electron scale to the very challenging precision of $0.02 \%$ (ATLAS Collaboration, 1999). The $C P$-violation experiments $\mathrm{KTeV}$ and NA48, which needed a similar knowledge of the electromagnetic energy scale to reconstruct with precision the $K_{S, L}^{0}$ decay vertices and therefore their relative production rate (which enters directly in the $\epsilon^{\prime} / \epsilon$ measurement), have indeed achieved such a precision.

The setting of the energy scale of the jet, i.e., inferring the original parton energy from the measured jet debris, is more complex than the setting of the electron scale since there are more numerous (and more difficult to control) sources of uncertainties. The calorimeters are calibrated by the use of test beams of single particles (electrons, pions) and not by jets: part of the jet energy can be carried away from neutrinos produced, for instance, in pion decays; part of the energy can be lost outside the cone that is used to collect the jet energy (this is usually the case if the original parton has irradiated one or more gluons); the rest of the event can contribute some energy inside the cone used to reconstruct the jet energy that needs to be subtracted; the calorimeter response to hadrons is usually noncompensated. Again, physics samples are necessary to set the final jet scale. The main samples used at hadron colliders are the associated production of a single jet with a photon or a $Z \rightarrow \ell \ell$. If there is only one jet and one boson in the event, then the boson and the jet must have equal and opposite momenta in the plane transverse to the beam axis [i.e., $\vec{p}_{T}(\gamma, Z)=-\vec{p}_{T}($ jet $\left.)\right]$. This is because the momenta of the interacting partons have negligible components transverse to the beam axis. The transverse momentum of the photon or $Z \rightarrow \ell \ell$ particle can be determined with high precision by using the electromagnetic calorimeter and the inner detector as described above. Therefore the jet scale can be obtained by requiring $\mid \vec{p}_{T}($ jet $)|=| \vec{p}_{T}(\gamma, Z) \mid$, that is, from the electromagnetic and tracker scales.

Figure 36 shows the inverse of the correction factor to the measured jet energy obtained in D0 as a function of energy by using a sample of $\gamma$-plus-one-jet events (Abbott et al., 1999). The experimental points can be fitted with the function $R_{\text {jet }}(E)=a+b \ln (E)+c \ln (E)^{2}$, where the logarithmic energy dependence reflects the logarithmic increase of the electromagnetic component of the hadronic cascade with energy (see Sec. III.A). The correction decreases with energy mainly because the calorimeter response to a jet becomes more compensating at high energy and because the energy losses in the dead material become smaller. The residual uncertainty on the jet scale after correction is at the level of $3 \%$ both in CDF and D0. It comes mainly from the limited statistics of the physics samples, from the subtraction of the underlying event and of the background, and from the corrections for the energy lost by longitudinal leakage, in the dead material, and out of the jet cone. The knowledge of the jet energy scale is the dominant systematic uncertainty in the measurement of the top mass at the Tevatron (Abbott et al., 1998; Abe et al., 1999), contrib- 
uting about $4 \mathrm{GeV}$ out of a total systematic error of about $5.5 \mathrm{GeV}$ in both $\mathrm{CDF}$ and D0.

Because of the larger event statistics, the LHC experiments hope to calibrate the jet energy scale with a precision of $\sim 1 \%$. This is important in order to measure the top mass to $\sim 1 \mathrm{GeV}$. In addition to the abovementioned physics samples, $W \rightarrow j j$ decays produced from top decays can also be used at the LHC to achieve this goal. Events due to $t \bar{t}$ production in which one top decays as $t \rightarrow b W \rightarrow b j j$ and one top as $t \rightarrow b W \rightarrow b \ell \nu$ are expected to be collected at the rate of one million per year of LHC operation and to have negligible backgrounds. The calorimeter jet scale can then be determined by requiring that the invariant mass of the two jets from $W \rightarrow j j$ decays in $t \bar{t}$ events be compatible with the $W$ mass. The latter should be known to better than $30 \mathrm{MeV}$ at the time of the LHC startup. The energy scale for $b$ jets will be established by using $Z+$ jet events, where the jet is tagged as a $b$ jet in the tracking system.

\section{LOW-TEMPERATURE CALORIMETERS}

\section{A. Introduction}

The extension of calorimetry into the domain of lowenergy deposits was motivated mostly by research in astroparticle physics. In these instruments the fundamental signal excitations-atomic transitions at the eV scale in classical calorimetry-are replaced by excitation and registration of phonons produced by the particle in suitable absorbers. The energies of these primary excitations (phonons or quasiparticles) are at a scale ranging from $\mu \mathrm{eV}$ (thermal phonons) to $\mathrm{meV}$ (nonequilibrium phonons or quasiparticles). Provided these phonon excitations can be measured above the thermal phonon noise, such calorimeters have the following advantages:

- considerably better energy resolution, because a given energy deposit produces many more signal quanta with correspondingly smaller statistical fluctuations;

- detection of processes with lower energy transfer because of reduced statistical fluctuations;

- detection of processes such as nuclear recoils that only or preferentially produce phonons but not scintillation or ionization signals.

A remarkable variety of methods have been explored in this young field of "phonon calorimetry." Common to all of them is the operation of the instrument at cryogenic temperatures $T<1 \mathrm{~K}$, such that a signal can be extracted above the thermal phonon noise. We shall describe three development efforts that have been shown or promise to address some of the most fundamental questions in astroparticle physics. We shall demonstrate the power of these devices with a few representative examples: relatively massive calorimeters for the search for rare events such as dark-matter particles, and singlephoton pixel detectors for nondispersive energy imaging.
Stock of these developments is regularly taken in annual workshops (Porter et al., 2002). Recent summaries provide very useful overviews (Booth, Cabrera, and Fiorini, 1996; Twerenbold, 1996; Pretzl, 2000).

\section{B. Main technologies}

The detectors discussed are all sensitive to phonon excitations; the application shapes the technical implementation. We discuss three developments that are representative of the field.

In "thermal detectors" the temperature rise is measured after the absorption of a particle inducing a new phonon distribution. The technical developments concentrate on the choice of the absorber and adequately sensitive thermometers. A second category, "phonon sensors," is based on superconducting tunneling junctions and responds to phonons through the destruction of Cooper pairs, giving rise to quasiparticles. These devices can be used as particle detectors for photons in the UV to $x$-ray energy range, combining the function of absorber and detector. Alternatively, they have found use as thermometers when coupled to a separate absorber. The third group, "superheated superconducting granules," combines again the function of absorber and detector: an energy deposit in such granules may drive them through the transition into the normal-conducting state, resulting in a change of the detectable magnetic properties.

\section{Thermal detectors}

The idea of measuring the temperature rise has been pursued for many decades (Pretzl, 2000). However, recent physics research motivations provided a new stimulus, and modern technology turned this idea into practical instruments.

The basic concept is beautifully simple. Consider an absorber with heat capacity $C(T)$ connected through a thermal conductance $G(T)$ to a reservoir at temperature $T_{B}$ (Fig. 37). An amount of energy $E$ deposited by an incident particle will produce a temperature rise $\Delta T$ :

$\Delta T=E / C(T) \cdot \exp (-t / \tau), \tau(T)=C(T) / G(T)$.

The idea works if $C(T)$ can be made small enough. This is the case for dielectric, diamagnetic substances and superconductors at $T \ll T_{c}$ for which only the lattice contributions to $C(T)$ are important and where

$$
C(T)=1944(m / M)\left(T / \theta_{\mathrm{D}}\right)^{3}\left[\mathrm{~J} \mathrm{~K}^{-1}\right] \text {. }
$$

Here $M, m$, and $\theta_{\mathrm{D}}$ are the molecular weight, the total mass, and the Debye temperature of the absorber, respectively. This $T^{3}$ dependence of the heat capacity allows a useful signal/noise ratio to be reached if the devices are operated at temperatures $T \lesssim 1 \mathrm{~K}$.

The above estimate of $\Delta T$ implies complete and relatively fast thermalization: a naive assumption. In general, energetic (tens of $\mathrm{meV}$ ) phonons are produced on a fast time scale $(\Sigma \mu \mathrm{s})$ but thermalize slowly. In addition, the absorber may contain metastable states or trapping 


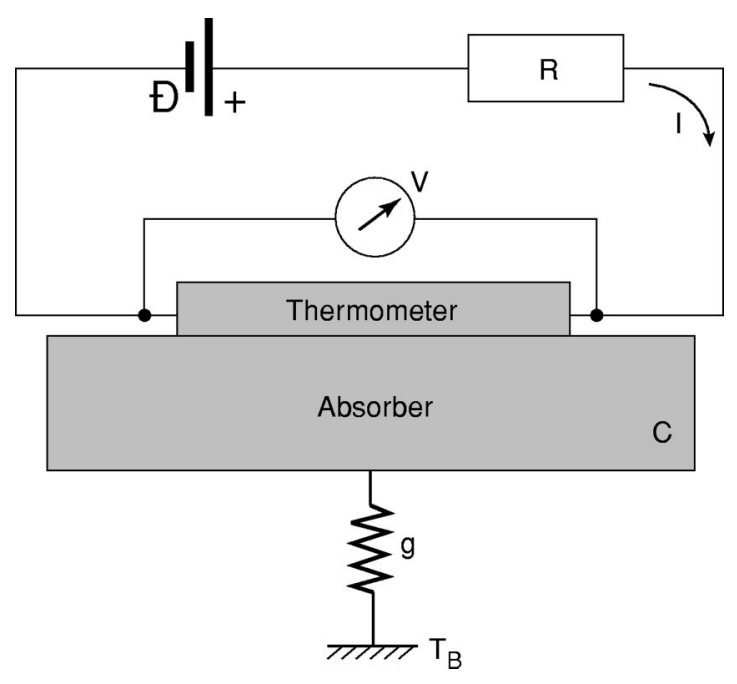

FIG. 37. Principle of a low-temperature calorimeter (Pretzl, 2000).

centers, resulting in further delayed thermalization. With this naive description an upper limit to the energy resolution $\sigma(E)$ can be estimated. Two components have to be considered: fluctuations in the intrinsic phonon background in the absorber and fluctuations of the number of phonon excitations.

The number of phonon modes is $C(T) / k_{\mathrm{B}}\left(k_{\mathrm{B}}\right.$ $=$ Boltzmann constant) with a mean energy $/$ mode of $k_{\mathrm{B}} T$. The corresponding fluctuations in this phonon gas in the absorber produce fluctuations in the phonon detector characterized by (Booth, Cabrera, and Fiorini, 1996)

$$
\sigma=\xi\left[k_{\mathrm{B}} T^{2} C(T)\right]^{1 / 2} \sim T^{5 / 2} .
$$

The factor $\xi$ expresses instrumental features (temperature sensor, thermal link, etc.) and in well-designed devices is typically in the range $\xi=1-2$. Values $\xi<1$ are achievable, however, with appropriate signal shaping (electrothermal feedback) (Irwin et al., 1995). The above equation gives the intrinsic instrument resolution due to the phonon noise in the absorber and is independent of energy. Numerically, these fluctuations are of the order of $\sigma(E) \sim 1 \mathrm{eV}$ for a germanium crystal of $10 \mathrm{~g}$ at $T=10 \mathrm{mK}$.

One should compare this resolution with the statistical fluctuations of the signal phonons. The general expression is $\sigma(E)=(\epsilon F E)^{1 / 2}$, where $\epsilon$ denotes the effective energy needed to produce an excitation and $F$ is the Fano factor previously described. Typical values in semiconductors or superconductors are $F \sim 0.1-0.2$. For the above example the phonon energy is $\epsilon \approx 1 \mathrm{meV}$ and an upper limit for $F$ is $F=1$. Therefore for a $1-\mathrm{keV}$ energy deposit one obtains $\sigma(E)=0.03 \mathrm{eV}$, which means that in this case the energy resolution would be limited by the intrinsic fluctuations. This is a naive lower limit, as in reality these detectors are also afflicted by the disease of invisible energy fluctuations: part of the absorbed energy may escape via radiative losses (electron and photon production); metastable states may trap phonons beyond the readout time; thermalization of the initial very nonthermal phonon spectrum is rarely completed during the readout time.

The excellent energy resolution of these detectors allows operation at a correspondingly low energy threshold, i.e., $E_{\text {th }} \sim$ few $\sigma(E)$. This is an important advantage in the search for (or study of) phenomena producing very small energy deposits.

A further unique feature of these phonon calorimeters is their sensitivity to low-ionizing or nonionizing particles. A typical application is the detection of nuclear recoils generated, for example, in the elastic scattering of dark-matter candidate particles that cannot be detected in ionization-sensitive calorimeters. This capability has been one of the major driving motivations in the development of these detectors.

Finally, a host of different materials are suitable as absorbers. This choice of materials opens the road to the study in novel ways of specific phenomena such as double- $\beta$ decay of selected isotopes (Alessandrello et al., 2000), dark-matter candidates, solar axions, etc. (Fiorini, 2000).

\section{Phonon sensors}

The phonon sensor is the "thermometer" needed for the operation of a bolometric calorimeter. In principle, any device with a property showing a sufficiently strong temperature dependence may be used. The typical rise time (determined by the detector physics) is $t_{r} \sim \mu \mathrm{s}$, and typical decay times are of the order of milliseconds.

In practice two types of device show a useful temperature behavior.

Doped semiconductor sensors are used as thermistors, either added as a separate component or integrated into the absorber through suitable local doping. A frequently used version is a germanium device in which uniform doping is achieved by thermal neutron irradiation (neutron-transmutation-doped germanium or Ge NTD), resulting in a resistance $R \sim T^{-\alpha}$, with $\alpha \simeq 1-10$, in the region around the operating point (Haller, 1995). The second popular sensor is the transition-edge sensor (TES). A superconducting metal electrode covers the absorber. These electrodes, which may be long, narrow, meandering strips of $\mathrm{Al}, \mathrm{Ti}$, or $\mathrm{W}$, or large electrodes of, for example, $\mathrm{Al}$, are operated at a temperature near the middle of the normal-superconductive transition. Absorption of the phonon pulse in these electrodes breaks the Cooper pairs and forms quasiparticles that subsequently release their energy and increase the resistance of the sensor. These sensors have typically a time response at the microsecond scale and allow, for example, interesting timing and spatial localization of the phonon pulse (Fig. 38). Dynamic range and energy linearity are improved by an ingenious electrothermal feedback providing dynamical biasing (Booth and Goldie, 1996; Cabrera et al., 2000).

Another frequently used sensor is the superconducting tunneling junction. In its basic execution, two superconductive films are separated by a thin (few nm thick) tunnel barrier. Phonon absorption breaks the Cooper 


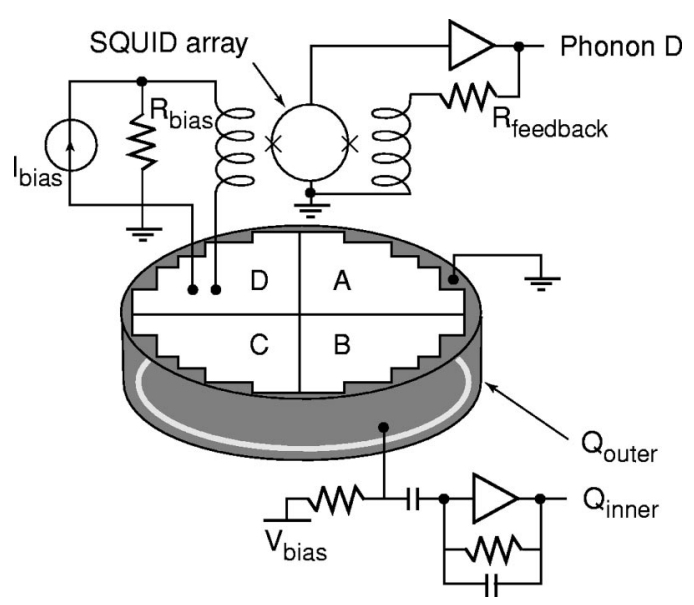

FIG. 38. Schematic diagram of a massive (few hundred grams) Ge detector for dark matter searches. Simultaneous measurements of phonons with transition-edge sensor and of the ionization charge provide the required background rejection $(\mathrm{Ca}-$ brera et al., 2000).

pairs and results in an increase of the tunneling current (excess carriers) on a fast time scale, ranging from nanosecond time constants in niobium to microsecond constants in hafnium (Booth, Cabrera, and Fiorini, 1996).

Alternatively, such excitations-quasiparticles-may be produced directly in the junction, for example, through the absorption of soft $\mathrm{x}$ rays, in which case the junction is an integrated absorber and detector (Peacock, 1999). These devices, when operated at sufficiently low temperature $T \leqq 0.1 T_{c}$, have few thermal carriers, such that the energy resolution $\sigma(E)$ is essentially limited by signal statistics $\sigma(E)=\left(\epsilon_{0} \cdot F E\right)^{1 / 2}$. The effective energy $\epsilon_{0}$ needed to produce a quasiparticle is $\epsilon_{0}$ $\sim 1.7 \Delta$, where $2 \Delta$ is the binding energy of a Cooper pair in the superconductor. The Fano factor is typically $F$ $\sim 0.2$. Such devices achieve resolutions of $\sigma(E)$ $\approx 20 \mathrm{eV}$ at $E \sim 1 \mathrm{keV}$ (Rando et al., 2000).

\section{Superheated superconducting granules}

Small (diameter $\sim 30 \mu \mathrm{m}$ ) superconducting granules (type-1 superconductors, such as $\mathrm{Sn}, \mathrm{Zn}, \mathrm{Al}$ ) are suspended in a dielectric matrix such as Teflon. The granules are operated in a metastable supercooled state at temperatures $T \sim 0.1 T_{c}$ in the presence of an external magnetic field. Particles depositing energy in the granules generate phonons that, through quasiparticle production, induce a phase transition to the normal conducting state with a time constant of $\sim 100 \mathrm{~ns}$. External pickup loops surrounding the detector volume of typically $10-50 \mathrm{~cm}^{3}$ sense the magnetic flux change due to this transition. Such detectors can be built with lowenergy thresholds (on the eV scale) and with excellent timing properties. A modular construction makes it possible to envisage detectors with active absorbers in the kilogram range (Pretzl, 2000).

\section{Representative applications}

We shall illustrate the range and potential of these instruments with a few representative applications.*

\section{Search for dark matter}

A considerable fraction of the nonvisible dark matter in the universe may be of nonbaryonic nature and may consist of as yet undiscovered weakly interacting massive particles. These particles may scatter elastically off the nuclei of the detector absorber material, leaving as sole signature nuclear recoils. Depending on the mass of the weakly interacting massive particles and on the detector nucleus, the average recoil energy varies in the $\mathrm{eV}$ to $\mathrm{keV}$ range. In conventional detectors such as scintillators, the signal from these very densely ionizing recoils is strongly suppressed ("quenched"), making phonon detection in low-temperature detectors an attractive recourse. Expected event rates are low ( $<1$ event per day and per kilogram of detector). The weakly interacting massive particles detectors have to be shielded from cosmic radiation and from local radioactive background. Additional background suppression needs to be achieved through, for example, the ability to discriminate between electron recoils (Compton scattering) and nuclear recoils.

A summary of several present dark-matter cryogenic experiments is given in Table IV. An example of the information obtained is given in Fig. 39, demonstrating the ability to discriminate among photons, electrons, and nuclear recoils (Hellmig et al., 2000).

\section{Neutrinoless double-beta decay}

Double-beta decay is a rare transition of an even-even nucleus $(A, Z)$ to its isobar $(A, Z+2)$ with the emission of two electrons and two neutrinos $(2 \nu$ double-beta decay), or with the neutrinoless emission of two electrons $(0 \nu$ double-beta decay). The latter transition would violate lepton number conservation and imply a nonzero neutrino mass.

In the search for $0 \nu$ double-beta decay it is advantageous, if not imperative, to use a large-mass, highresolution detector that contains the $0 \nu$ double-betadecay candidate isotope. With conventional detectors this technique is practically limited to ${ }^{76} \mathrm{Ge}$. Lowtemperature detectors offer a considerably larger choice of isotopes. At present, the best limits are obtained by studying the isotope ${ }^{130} \mathrm{Te}$, using $\mathrm{TeO}_{2}$ bolometers with neutron-transmutation-doped Ge sensors and with a total mass of almost $7 \mathrm{~kg}$. The success of this method, pioneered by the Milano group, has generated an ambi-

\footnotetext{
*Editor's note: Another application that has appeared after the present review was written is the detection and measurement of very-long-lifetime radioactivity [de Marcillac, Coron, Dambler, Leblanc, and Moalic, Nature 422, 876 (2003)]. The detector used a scintillating bolometer operating at a temperature $T=20 \mathrm{mK}$ with a sensor of neutron-transmuted doped germanium.
} 
TABLE IV. Weakly interacting massive particle searches with cryogenic calorimeters.

\begin{tabular}{|c|c|c|c|c|}
\hline Experiment & Location & Absorber & Readout technique & Reference \\
\hline CDMS & Stanford, USA & germanium, silicon & $\begin{array}{l}\text { phonon TES; } \\
\text { ionization }\end{array}$ & Hellmig et al., 2000 \\
\hline CRESST & Gran Sasso, Italy & sapphire (total>1 kg) & $\begin{array}{l}\text { superconducting } \\
\text { phase transition } \\
\text { thermometers }\end{array}$ & Sisti et al., 2000 \\
\hline EDELWEISS & Fréjus, France & germanium (70 g) & $\begin{array}{l}\text { phonon and } \\
\text { charge readout }\end{array}$ & Chardin et al., 2000 \\
\hline CUORICINO & Gran Sasso, Italy & $\mathrm{TeO}_{2}(6.8 \mathrm{~kg})$ & NTD Ge thermistor & Alessandrello et al., 2000 \\
\hline ORPHEUS & Bern, Switzerland & $\begin{array}{l}\text { superconducting } \\
\text { Sn grains } \\
\text { (1 kg, under construction) }\end{array}$ & 50 pickup coils & van den Brandt, 2000 \\
\hline ROSEBUD & Canfranc, Spain & sapphire $(100 \mathrm{~g})$ & NTD Ge thermistor & Cebrian et al., 2000 \\
\hline TOKYO & $\begin{array}{l}\text { Nokogiri-yama } \\
\text { Japan }\end{array}$ & $\mathrm{LiF}(\sim 170 \mathrm{~g})$ & NTD Ge thermistor & Ootani et al., 1999 \\
\hline
\end{tabular}

tious proposal for a cryogenic detector laboratory with detectors in the one-ton range (Fiorini, 2000).

\section{Microcalorimeters for x-ray astronomy}

The outstanding energy resolution of low-temperature calorimeters suggests replacing dispersive spectroscopy (e.g., a Bragg crystal spectrometer) with the wideband large acceptance of bolometers, as pioneered by McCammon et al. (1993). This group has developed an array of $36 \mathrm{x}$-ray detectors, each $0.32 \times 1.23 \mathrm{~mm}^{2}$ in size, consisting of $\mathrm{HgTe}$ absorbers with implanted silicon thermistors. The calorimeter, operated at $60 \mathrm{mK}$, shows an energy resolution $\sigma(E) \approx 4-5 \mathrm{eV}$ for $6-\mathrm{keV}$ x-ray photons. Much of the thrust of the research and development effort is directed towards larger arrays consisting of 1000 pixels and cryogenic technology suitable for space-borne operation (Kelley et al., 2000).

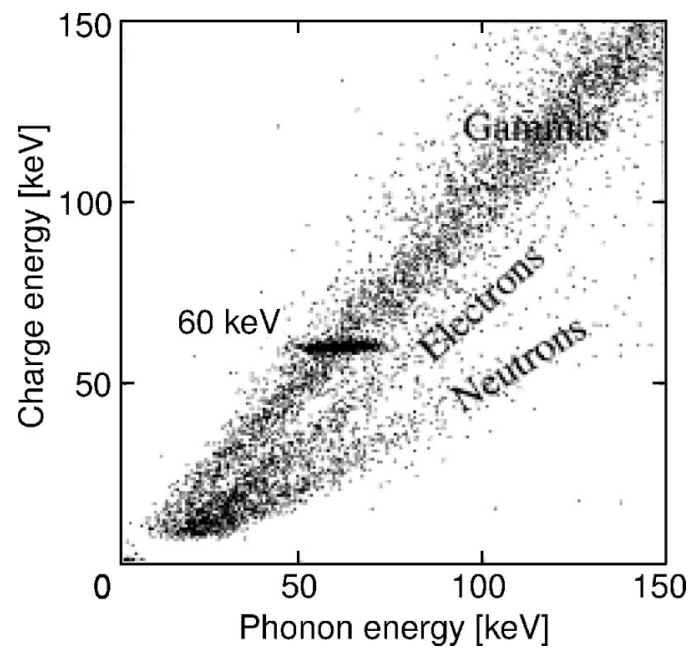

FIG. 39. Charge and phonon energy from ${ }^{14} \mathrm{C}$ electrons, ${ }^{241} \mathrm{Am}$ $60-\mathrm{keV}$ photons, and ${ }^{252} \mathrm{Cf}$ neutrons measured in a Ge detector equipped with a transition-edge phonon sensor (Hellmig et al., 2000).
4. Superconducting tunneling junctions for ultraviolet to infrared spectroscopy in astronomy

As in the previous example, detectors are being developed for dispersive spectroscopy in the wavelength region of $0.5 \mathrm{~nm}$ to $\sim 2 \mu \mathrm{m}$. For these energy deposits, arrays of superconducting tunneling junctions serve the dual function of absorber and detector. The number of excess carriers $N_{0}$ produced by the photon absorption as a function of the wavelength $\lambda$ is (Peacock, 1999)

$$
N_{0}(\lambda) \sim 7 \times 10^{5} /\left[\lambda(\mathrm{nm}) \Delta\left(T / T_{c}\right)(\mathrm{meV})\right]
$$

In a typically used junction material such as tantalum, $N_{0}(\lambda) \sim 10^{6}$ at $\lambda \approx 1 \mathrm{~nm}$. The variance of $N_{0}$, determining the spectroscopic resolution, depends on the fraction of phonons with energy $\Omega>2 \Delta$, the detectable energy which can break the Cooper pairs, and on the temperature-dependent superconductor band gap $\Delta$. Expressed in terms of wavelength resolution

$$
d \lambda(\mathrm{nm}) \sim 2.8 \times 10^{-3} \lambda^{3 / 2}[F \Delta]^{1 / 2},
$$

where $F \sim 0.2$ (Peacock, 1999). Figure 40 summarizes the resolution for a number of superconductors; the measurements shown for niobium and tantalum superconducting tunneling junctions indicate that it is possible to approach the theoretical limit of resolution. The potential of hafnium superconducting tunneling junctions is attractive but requires operation in the $10-\mathrm{mK}$ domain. This technology has been developed to the point that a demonstrator 36-pixel-array niobium-based superconducting tunneling junction is presently being operated at the William Herschel Telescope on La Palma (Canary Islands, Spain) (Rando et al., 2000). Arguably, this technique will revolutionize astronomy. 


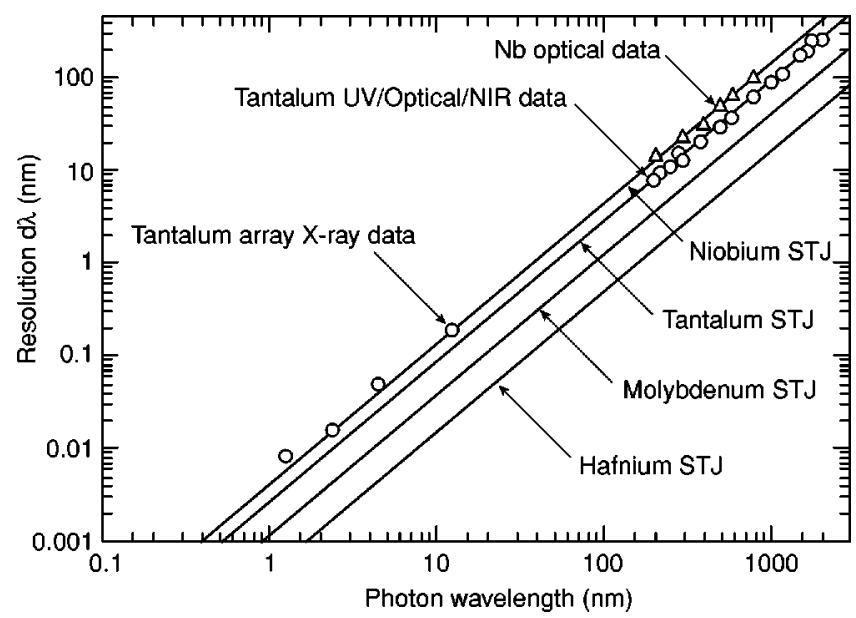

FIG. 40. Theoretical limit of resolution for several superconductors as a function of wavelength, compared to experimental data for $\mathrm{Nb}$ and $\mathrm{Ta}$ superconducting tunneling junctions. Experimental measurements obtained with niobium and hafnium are also shown (Peacock, 1999).

\section{CITIUS, ALTIUS, FORTIUS ${ }^{1}$}

\section{A. Introduction}

Every few seconds a particle with an energy close to $10^{20} \mathrm{eV}$ hits the Earth from outer space, shedding light on a major enigma in astroparticle physics: How and where are these particles produced? Is it true that they must be of galactic origin, because particles with energies $E \gtrsim 10^{20} \mathrm{eV}$ have collision cross sections with the 2.7-K microwave background so large as to limit their range $R$ to $R \leqq 50 \mathrm{Mpc}$, the Greisen-Zatsepin-Kuzmin (GZK) cutoff (Greisen, 1966; Zatsepin and Kuzmin, 1966), or are these particles messengers of the next revolution in astroparticle physics?

Equally tantalizing is the observation of very-highenergy gamma rays with $E \lesssim 1 \mathrm{TeV}$. The measurement of their flux, point of origin, and energy spectra provides clues about very energetic electromagnetic and nuclear processes. These gamma rays may be emitted in jets emerging from active galactic nuclei, from the environment surrounding rapidly spinning neutron stars, or from supernovae remnants. Very-high-energy gamma astronomy probes the origin of these energetic beams and the medium in which these gamma rays are generated (Hoffman, Sinnis, and Fleury, 1999).

Even more fleeting are signals produced by extraterrestrial neutrinos. Neutrino astronomy is yet another nascent branch of astronomy opening a totally new window to our universe. Very-high-energy neutrinos may actually signal relics of the Big Bang, e.g., of the phase transition at the grand unified theory scale; supersymmetric neutralinos may aggregate in the center of our galaxy and annihilate with accompanying neutrino emission. Much closer to us, neutrino emission from the Sun

${ }^{1}$ Plagiarized from V. Trimble's article in the SLAC Beamline, Vol. 28, No. 3, p. 18 (1998).

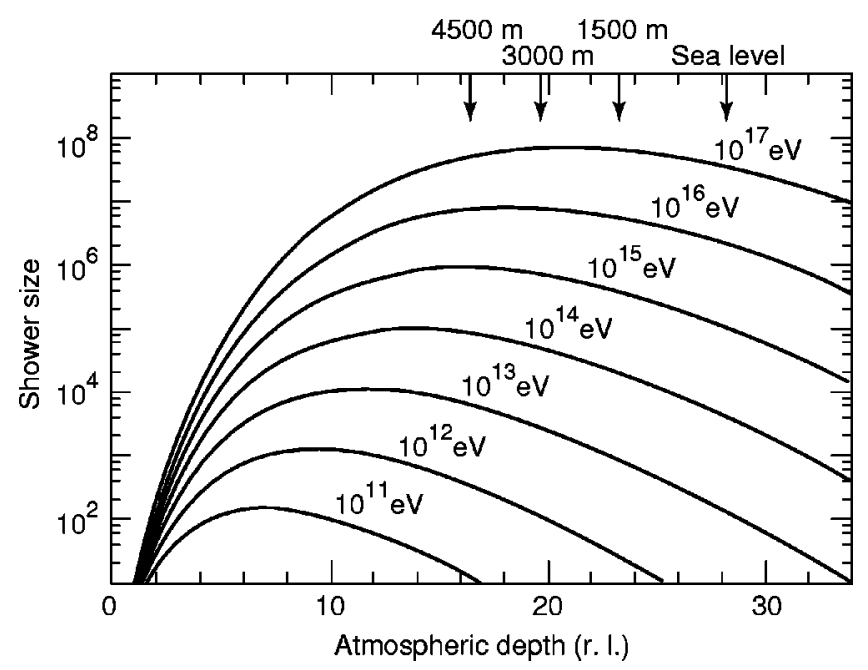

FIG. 41. Longitudinal shower developments (in radiation lengths) initiated by high-energy photons in the atmosphere. The shower size refers to the number of electrons and positrons present in the shower at a given shower depth (Cronin, Gibbs, and Weekes, 1993).

remains of fundamental significance.

Common to almost all of these investigations is the rarity of the detected signals, requiring instrumentation at an "astronomical" scale. For very-high-energy photon and hadron detection the Earth's atmosphere is, however, a precious gift of nature. At sea level the atmosphere represents an absorber of approximately 28 radiation lengths and almost 17 collision lengths: a wonderful calorimeter that is homogeneously sensitive if appropriately instrumented. Only for neutrino detection is even more absorber mass required: sea water and the Antarctic ice cap are being instrumented for neutrino astrophysics.

In the following section we shall discuss atmospheric calorimeters, their performance, and current instrumental approaches. This is followed by a brief account of "gigaton" calorimeters for neutrino astronomy.

\section{B. Atmospheric calorimeters}

At sea level the Earth's atmosphere has a pressure of $1030 \mathrm{~g} / \mathrm{cm}^{2}$. Its composition of $20.93 \% \mathrm{O}_{2}, 78.10 \% \mathrm{~N}_{2}$, and $0.93 \% \mathrm{Ar}$ translates into a radiation length of $X_{0}$ $=36.66 \mathrm{~g} \mathrm{~cm}^{-2}$, i.e., a total of $28.1 X_{0}$ (Tsai, 1974). The collision length is $\lambda=62.0 \mathrm{~g} \mathrm{~cm}^{-2}$, giving a total of $16.6 \lambda$ (Particle Data Group, 2002). For air the critical energy is $\varepsilon=85.9 \mathrm{MeV}$ and the Molière radius $R_{M}=9.05 \mathrm{~g} \mathrm{~cm}^{-2}$.

With these parameters the shower profiles can be evaluated. One such example is shown in Fig. 41. Photon showers with energies up to $E_{\gamma} \gtrsim 10 \mathrm{TeV}$ are fully contained. If the energy containment is incomplete, the usual leakage corrections need to be made. The "tail" of such showers can be analyzed with ground-based detectors and the energy estimated.

Ingenious approaches have been developed to instrument this homogeneous absorber. Relevant performance parameters for these atmospheric calorimeters are as follows: 
TABLE V. Parameters of representative facilities for gamma- and cosmic-ray observation.

\begin{tabular}{|c|c|c|c|c|c|c|c|}
\hline Facility & $\begin{array}{l}\text { Year of } \\
\text { first } \\
\text { operation }\end{array}$ & Depth & Size & $\begin{array}{l}\text { Muon } \\
\text { detector } \\
\text { size }\end{array}$ & $\begin{array}{l}\text { Instrumentation } \\
\text { technique }\end{array}$ & $\begin{array}{l}\text { Angular } \\
\text { resolution }\end{array}$ & $\begin{array}{l}\text { Energy } \\
\text { range }\end{array}$ \\
\hline $\begin{array}{l}\text { Haverah } \\
\text { Park }^{\mathrm{a}}\end{array}$ & 1968 & $1010 \mathrm{~g} / \mathrm{cm}^{2}$ & $C=12 \mathrm{~km}^{2}$ & & Cherenkov tanks & $1^{\circ}$ & $\begin{array}{l}60000 \mathrm{TeV} \\
\sim 10^{8} \mathrm{TeV}\end{array}$ \\
\hline Whipple ${ }^{\text {b }}$ & 1968 & $875 \mathrm{~g} / \mathrm{cm}^{2}$ & $R=78.6$ & & Cherenkov telescope & $0.15^{\circ}$ & $0.1-10 \mathrm{TeV}$ \\
\hline Yakutsk $^{\mathrm{c}}$ & 1973 & $1020 \mathrm{~g} / \mathrm{cm}^{2}$ & $C=20 \mathrm{~km}^{2}$ & $\begin{array}{l}R=292 \\
\mathrm{~m}^{2}\end{array}$ & $\begin{array}{l}\text { Air shower array }+ \\
\text { Cherenkov air light det. }+ \\
\text { muon counter }\end{array}$ & & $\begin{array}{l}10^{5} \mathrm{TeV}- \\
\sim 10^{8} \mathrm{TeV}\end{array}$ \\
\hline $\mathrm{AKENO}^{\mathrm{d}}$ & 1975 & $920 \mathrm{~g} / \mathrm{cm}^{2}$ & $C=20 \mathrm{~km}^{2}$ & $\begin{array}{l}R=225 \\
\mathrm{~m}^{2}\end{array}$ & $\begin{array}{l}\text { Air shower }+ \\
\text { muon counter }\end{array}$ & $1^{\circ}$ & $\begin{array}{l}1000 \mathrm{TeV} \text { and } \\
\text { above }\end{array}$ \\
\hline $\begin{array}{l}\text { HiRes } \\
\text { Fly's Eye }\end{array}$ & 1981 & $860 \mathrm{~g} / \mathrm{cm}^{2}$ & $R=182 \mathrm{~cm}^{2}$ & & Fluorescent light detector & $1^{\circ}$ & $\begin{array}{l}10^{5} \mathrm{TeV}- \\
32107 \mathrm{TeV}\end{array}$ \\
\hline CASA-MIA $^{\mathrm{e}}$ & 1990 & $870 \mathrm{~g} / \mathrm{cm}^{2}$ & $\begin{array}{l}A=6000 \mathrm{~km}^{2} \mathrm{sr} \\
C=230000 \mathrm{~m}^{2}\end{array}$ & $\begin{array}{l}R=2560 \\
\mathrm{~m}^{2}\end{array}$ & $\begin{array}{l}\text { Air shower array }+ \\
\text { muon scintillator }\end{array}$ & $1^{\circ}$ & $\begin{array}{l}70 \mathrm{TeV} \text { and } \\
\text { above }\end{array}$ \\
\hline $\operatorname{AGASA}^{\mathrm{f}}$ & 1990 & $920 \mathrm{~g} / \mathrm{cm}^{2}$ & $\begin{array}{l}R=1600 \mathrm{~m}^{2} \\
C=100 \mathrm{~km}^{2}\end{array}$ & $\begin{array}{l}C=100 \\
\mathrm{~km}^{2}\end{array}$ & $\begin{array}{l}\text { Air shower array }+ \\
\text { muon counter }\end{array}$ & $1^{\circ}$ & 310-26107 TeV \\
\hline & & & $\begin{array}{l}A=2.6 \times 10^{10} \mathrm{~km}^{2} \mathrm{sr} \\
R=111 \times 2.2 \mathrm{~m}^{2}\end{array}$ & & & & \\
\hline Hegra & 1996 & $800 \mathrm{~g} / \mathrm{cm}^{2}$ & $\begin{array}{l}C=32400 \mathrm{~m}^{2} \\
R=972 \mathrm{~m}^{2}\end{array}$ & $\begin{array}{l}R=272 \\
\mathrm{~m}^{2}\end{array}$ & $\begin{array}{l}\text { Scintillator counter }+ \\
\text { open Cherenkov } \\
\text { counter }+ \\
\text { Cherenkov telescope }+ \\
\text { Geiger tower }\end{array}$ & $0.2^{\circ}$ & $1-10000 \mathrm{TeV}$ \\
\hline Tibet $\mathrm{Ag}^{\mathrm{g}}$ & 1996 & $600 \mathrm{~g} / \mathrm{cm}^{2}$ & $\begin{array}{l}C=36900 \mathrm{~m}^{2} \\
R=147 \mathrm{~m}^{2}\end{array}$ & & Air shower array & $0.9^{\circ}$ & $3-100 \mathrm{TeV}$ \\
\hline KASKADE $^{\mathrm{h}}$ & 1997 & & $\begin{array}{l}C=40000 \mathrm{~m}^{2} \\
R=1450 \mathrm{~m}^{2}\end{array}$ & $\begin{array}{l}C=40000 \\
\mathrm{~m}^{2} \\
R=1450 \\
\mathrm{~m}^{2}\end{array}$ & $\begin{array}{l}\text { Air shower array } \\
\quad \text { for e, } \gamma, \mu \\
320 \mathrm{~m}^{2} \text { hadron calorimeter }\end{array}$ & & $100-10^{5} \mathrm{TeV}$ \\
\hline Magic & 2001 & $800 \mathrm{~g} / \mathrm{m}^{2}$ & $R=234 \mathrm{~m}^{2}$ & & Cherenkov telescope & $0.02^{\circ}$ & $0.015-50 \mathrm{TeV}$ \\
\hline Auger & 2003 & $880 \mathrm{~g} / \mathrm{cm}^{2}$ & $\begin{array}{l}C=2 \times 3000 \mathrm{~km}^{2} \\
A=2 \times 7500 \mathrm{~km}^{2} \mathrm{sr} \\
R=16000 \mathrm{~m}^{2}\end{array}$ & & $\begin{array}{l}\text { Cherenkov tanks } \\
\text { Fluorescent detector }\end{array}$ & $0.3^{\circ}$ & $\begin{array}{l}10^{7} \mathrm{TeV} \text { and } \\
\text { above }\end{array}$ \\
\hline Veritas $^{\mathrm{i}}$ & 2004 & $875 \mathrm{~g} / \mathrm{cm}^{2}$ & $\begin{array}{l}R=7 \times 78.64 \mathrm{~m}^{2} \\
C=40000 \mathrm{~m}^{2}\end{array}$ & & Cherenkov telescope & $0.015^{\circ}$ & $0.015-50 \mathrm{TeV}$ \\
\hline
\end{tabular}

${ }^{\mathrm{a}}$ Hillas et al. (1971).

${ }^{\mathrm{b}}$ Mohanty et al. (1998).

${ }^{c}$ Yakutsk Extensive Air Shower Array (2001).

${ }^{\mathrm{d}}$ Takeda et al. (1999).

${ }^{\mathrm{e}}$ Cronin, Gibbs, and Weekes (1993).

- the energy threshold for particle detection;

- the systematic error on the energy scale;

- the energy resolution;

- their potential to discriminate between different particles.

These performance parameters depend on the concept chosen and on the technical sophistication of the instrumentation. A recent review emphasizing gamma-ray astronomy is given by Hoffman, Sinnis, and Fleury (1999).

In the following, three experimental techniques are discussed, and major representative facilities are listed in Table V.

\author{
${ }^{\mathrm{f}}$ Hayashida et al. (1994). \\ ${ }^{\mathrm{g}}$ Amenomori et al. (2000). \\ ${ }^{\mathrm{h}}$ Kampert et al. (2001). \\ ${ }^{\mathrm{i}}$ Bond (2000).
}

One approach, very actively developed during the past 20 years, is tailored to explore the energy window between space-based detectors, rate limited to below $E$ $\lesssim 10 \mathrm{GeV}$, and ground-based systems, limited by signalto-noise ratio to $E \gtrsim 300 \mathrm{GeV}$. The energy information is obtained from the Cherenkov light produced by the passage of the relativistic cascade particles through the atmosphere (Fig. 42). The lateral distribution of this "Cherenkov shower" is narrowly concentrated around the shower axis: therefore with appropriate instrumentation this "lightning rod" can be observed in the night sky.

Modern instruments exploiting this atmospheric Cher- 


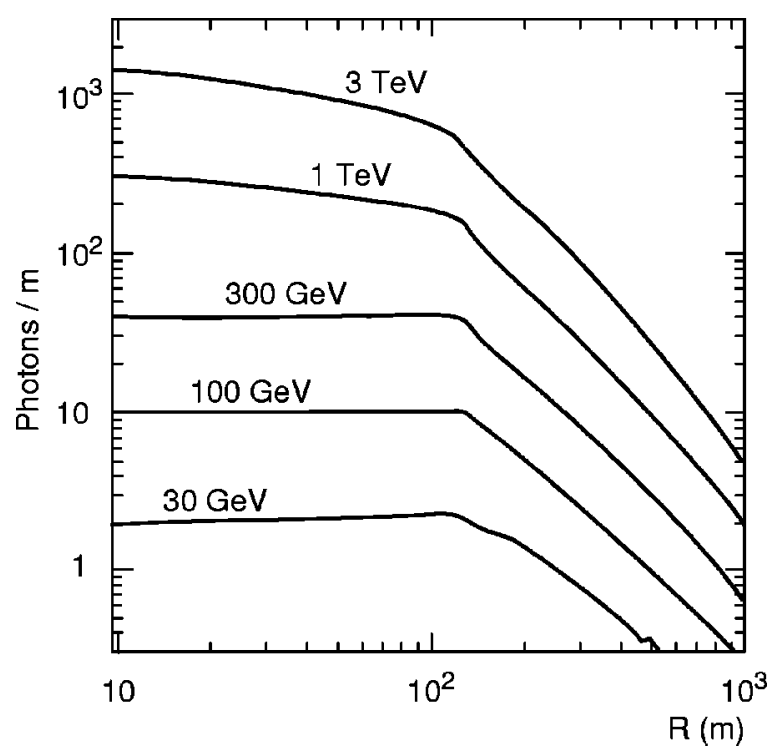

FIG. 42. Cherenkov photon density between 300 and $600 \mathrm{~nm}$ as a function of distance from the shower impact point (impact parameter $R$ ), for various $\gamma$-ray energies, as seen at $2 \mathrm{~km}$ above sea level for vertical showers [adapted from Hoffman, Sinnis, and Fleury (1999)].

enkov technique have imaging quality, collecting the Cherenkov light and imaging it onto a "pixel" array of Cherenkov photon detectors, providing direction, shape, energy, and particle-type information (Fig. 43). Key to the success of the imaging atmospheric Cherenkov technique is the suppression of background, dominated by the light from the night sky and cosmic-ray-induced hadronic showers. The latter source is potentially devastating because at energies of interest $(10 \mathrm{GeV}$ to multi$\mathrm{TeV}$ ) they dominate over gamma rays by factors of $10^{3}-10^{4}$. These cosmic hadrons can be rejected (Fig. 44) through directional and shower-shape analysis (Mohanty et al., 1998). The imaging atmospheric Cherenkov technique allowed the recent observation of $\gamma$ point sources, Markarian 421, Markarian 501, and the BL Lac object 1ES 2344+ 514 (Hoffman, Sinnis, and Fleury, 1999).

In a second approach, pioneered by Cassiday and col-

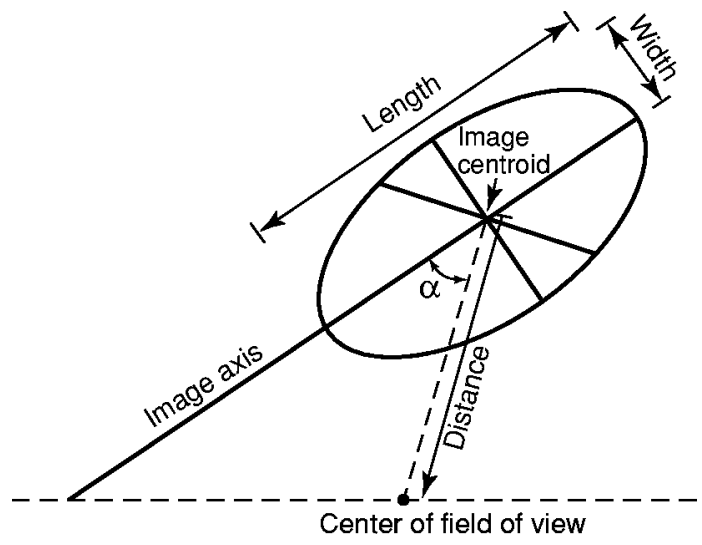

FIG. 43. Image parameters used in the analysis of imaging atmospheric Cherenkov technique information (Mohanty et al., 1998).
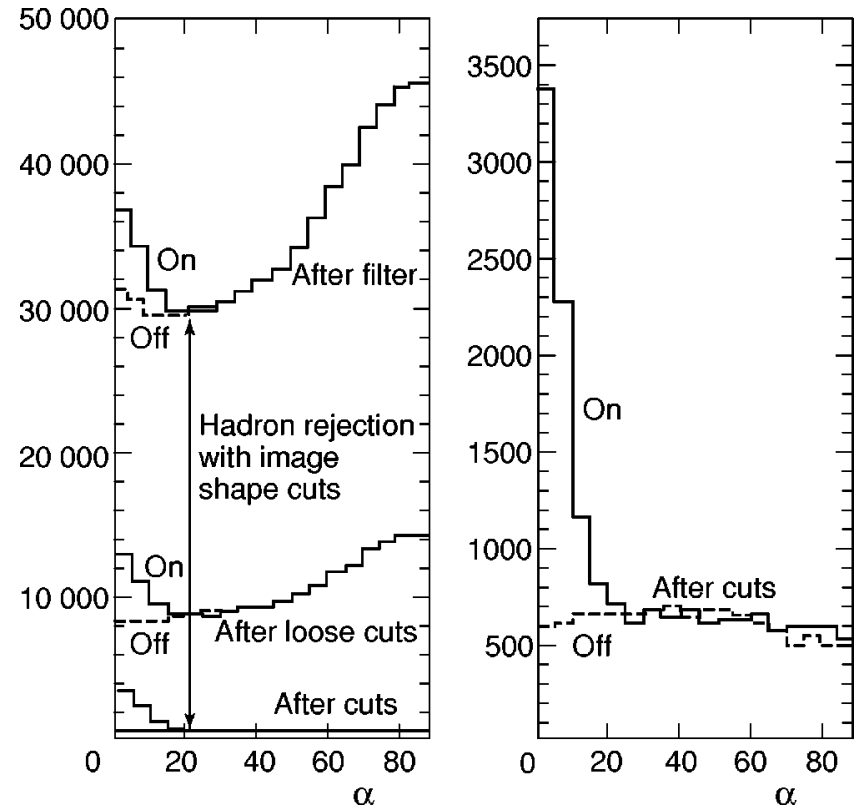

FIG. 44. Suppression of hadronic background through image shape analysis of the extragalactic Makarian 501 point source, observed by the HEGRA telescope. The right figure shows on an expanded scale the $\gamma$ /hadron ratio after optimized cuts, with the $\gamma$ signal concentrated at values $\alpha<10^{\circ}$. "ON" ("OFF") refers to ON (OFF) -source data (Barrio et al., 1998).

laborators (1985), the fluorescence excited by the passage of the shower particles in the atmosphere provides the measure of the particle's energy. The observatory consists of an array of telescope mirrors that look into the night sky and focus the fluorescent light onto an array of photomultipliers. Compared to the atmospheric Cherenkov technique, this instrument observes a very large volume of the atmosphere-the acceptance is $\sim 6000 \mathrm{~km}^{2} \mathrm{sr}-\mathrm{in}$ a grid of $\sim 1^{\circ} \times 1^{\circ}$, hence the name of Fly's Eye. This technique allows a measurement of the longitudinal shower profile and of the shower maximum, which is sensitive to the composition of the cosmic rays. It is one of the facilities having recorded the highestenergy cosmic rays to date (Bird et al., 1995). The original array has now been replaced with a novel facility, HiRes, to address the enigmatic energy domain around the GZK cutoff (Booth and Goldie, 1996; Boyer et al., 2002).

The oldest (Murzin, 1967) of the three methods, still widely used, is the air shower technique. It uses groundbased arrays of detectors to record the tails of particle cascades initiated by sufficiently energetic primaries. As such it is a "tail catcher" with only one absorber layer (the atmosphere) and one detector layer. It works only for sufficiently energetic primaries, for which a large number of shower particles "leak" into the instrumentation layer.

The development of this technique (Cronin, Gibbs, and Weekes, 1993) aims at

- increased sensitivity towards higher energies;

- better understanding of the absolute energy scale; 
TABLE VI. Neutrino observatories: present and near future.

\begin{tabular}{|c|c|c|c|c|}
\hline Detector & $\begin{array}{l}\text { Year of first } \\
\text { operation }\end{array}$ & $\begin{array}{l}\text { Technique } \\
\text { and location }\end{array}$ & $\begin{array}{l}\text { Instrumented } \\
\text { active volume }\left(\mathrm{m}^{3}\right)\end{array}$ & Reference \\
\hline Lake Baikal & 1998 & $\begin{array}{l}192 \text { photomultipliers ( } 37 \mathrm{~cm} \text { diam.) } \\
\text { Lake Baikal at } 1.1-\mathrm{km} \text { depth }\end{array}$ & $\lesssim 3 \times 10^{5}$ & Balkanov, 2000 \\
\hline Nestor & under construction & $\begin{array}{l}168 \text { photomultipliers ( } 15 \text { in. diam.) } \\
\text { Mediterranean at 3.8-km depth }\end{array}$ & $\sim 6 \times 10^{5}$ & Griedler, 2001 \\
\hline Antares & in preparation & $\begin{array}{l}1170 \text { photomultipliers } \\
\text { Mediterranean at 2.4-km depth }\end{array}$ & $\sim 2 \times 10^{7}$ & Aslanides et al., 1999 \\
\hline Amanda & 1998 & $\begin{array}{l}302 \text { photomultipliers ( } 8 \text { in. diam.) } \\
\text { Antarctic ice at } 1.5-1.9-\mathrm{km} \text { depth }\end{array}$ & $\leqq 2 \times 10^{6}$ & Hill et al., 2001 \\
\hline
\end{tabular}

— identification of the primary composition;

— better signal-to-background ratio.

Besides increasing the detection area to increase the energy reach, the angular resolution is at a premium to discriminate, for example, $\gamma$ sources against the diffuse hadronic flux.

Typical values for the angular resolution $\sigma_{\theta}$ are at the level of $0.3^{\circ}-3^{\circ}$, which is achieved by measuring the arrival time of the air shower in the detector array. It is good enough to permit a direct calibration of this parameter by observing, for example, the reduction of the cosmic-ray flux due to the shadowing of the Moon (Hoffman, Sinnis, and Fleury, 1999).

This technique, as well as the HiRes approach, lends itself to the exploration of the high-energy frontier beyond the GZK cutoff. AGASA, the largest of the operational air shower technique arrays, has reported several events with energies beyond the GZK cutoff (Takeda et al., 1998).

The most ambitious project, presently under construction, is the Auger observatory, which will measure over an area of $3000 \mathrm{~km}^{2}$. It is a hybrid design combining the advantages of ground arrays (100\% duty cycle, muon/ electromagnetic ratio sensitivity) and of the fluorescent detector technique (complementary energy estimation, measurement of shower shapes). When operational, the array will collect $\sim 30$ events/year with energy $E$ $>10^{20} \mathrm{eV}$ (Pryke, 1998).

\section{Setting the energy scale}

The imaging atmospheric Cherenkov technique must rely on "dead-reckoning" of the energy scale through a simulation from the first interaction to the photon detection. Fortunately, in the energy range of the imaging atmospheric Cherenkov technique the physics simulation of the shower development can at least in principle be benchmarked by experimental measurements. Nevertheless, even very systematic studies (Mohanty et al., 1998) do not completely address the uncertainties affecting the absolute scale. Circumstantial evidence, e.g., the $\gamma$ rate from the Crab Nebula measured by different observatories, indicates an impressively small absolute scale error at the $20 \%$ level (Aharonian et al., 2000). Through recent improvements the imaging atmospheric Cherenkov technique has gained enough sensitivity to measure isolated muons: it should be possible to tie the muon energy loss to the energy scale and therefore improve on the absolute calibration (Hoffman, Sinnis, and Fleury, 1999).

Knowledge of the absolute energy scale is a central issue also for Fly's Eye and HiRes. At the extreme energies probed by these observatories the hadroninduced showers transform almost completely ( $\gtrsim 90 \%)$ into electromagnetic energy (Sec. III.A), which provides a reliable lower limit to the energy estimate. Corrections for incomplete containment ( $\sim 50 \%)$ may be more critical, as are uncertainties in the light attenuation in the atmosphere. The uncertainty in the energy scale is estimated at the 30\% level (Seman, 2001).

\section{Energy resolution}

In present applications of the imaging atmospheric Cherenkov technique the energy resolution is not a driving design parameter. As an example, the Whipple Observatory, which has made major contributions to veryhigh-energy $\gamma$ studies, has a constant energy resolution of $\sigma / E \sim 70 \%$, over the energy range $300<E_{\gamma}$ $<5000 \mathrm{GeV}$ (Mohanty et al., 1998). Better performance, $\sigma / E \sim 0.2$ at $100 \mathrm{GeV}$, weakly improving with energy (Konopelko et al., 1999), is quoted for the HEGRA array of telescopes. Constant energy resolution in calorimeters is of course an indication that the performance is dominated by instrumental features masking the intrinsic detector performance. It is the next generation of these remarkable devices that will be instrumented to reach energy resolutions described by $\sigma / E \sim E^{-l / 2}$, typical of calorimeters (Martinez, 1998).

\section{Deep-water calorimeters}

The most massive calorimeters are being developed to detect extraterrestrial neutrinos. These observatories are optimized to instrument large areas/volumes, for example, in deep seas or in the Antarctic ice cap. They detect the Cherenkov light originating from charged secondary particles produced in the $\nu$ interaction with the absorber.

In presently operational devices (Table VI), the predominantly detected signal is produced by upward-going muons from charged-current neutrino interactions in the 
material below the detector. Such detectors may also study $\nu_{\mu}$ 's produced in the upper atmosphere. Our universe is also less opaque to $\nu$ 's than to protons such that $\nu$ eyes can look beyond the GZK cutoff. The present generation of modest-sized prototypes aims to develop the methods of $\nu$ astronomy at energies comparable to those of the highest-energy gammas or hadrons observed. Successful development of gigaton-sized $\nu$ observatories during this century might contribute results as significant as those obtained by electromagnetic astronomy to date. Such facilities are being developed by the NESTOR (Griedler, 2001) and ANTARES (Aslanides et al., 1999) Collaborations. Recent summaries of some of the major projects can be found in Spiering (1999) and Cecchini (2001).

A further "extreme" route is being pursued by the AMANDA Collaboration (Hallgren, 2002), which has instrumented approximately $10^{7} \mathrm{~m}^{3}$ of Antarctic ice with strings of photomultipliers at a depth of 1550-2350 $\mathrm{m}$ below the surface. With the present detector area of $\sim 30000 \mathrm{~m}^{2}$ atmospheric neutrinos have been measured at the expected level. The Collaboration plans to extend this technology and build an "ICECUBE" with $1-\mathrm{km}^{2}$ detector area, reaching sensitivities to explore point sources (Hill et al., 2001).

\section{CONCLUSIONS}

The story of modern calorimetry is a textbook example of physics research driving the development of an experimental method. The long quest for precision electron and photon spectroscopy explains the remarkable progress in new instrumentation techniques, for both sampling and homogeneous electromagnetic detectors. The study of jets of particles as the macroscopic manifestation of quarks has driven the work on hadronic calorimeters. These techniques have been developed to meet experimental needs at today's and tomorrow's accelerators.

New frontiers, e.g., in astroparticle physics, have led to the innovative development of low-temperature devices and to the instrumentation of ever larger volumes.

New measurement techniques open the way to fundamental discoveries but also lead to applications in other areas. On both accounts calorimetry already has a distinguished record. If the breadth and intensity of research and development in calorimetry is a yardstick, we should expect many more fundamental discoveries and fascinating applications.

\section{ACKNOWLEDGMENTS}

We are grateful to several colleagues, A. Ferrari, D. Fournier, A. Giuliani, K. Pretzl, J. Ranft, T. Virdee, and R. Wigmans, who have provided very helpful comments and information. We thank the CERN DTP group for their patience and professional help with the manuscript.

\section{REFERENCES}

Abashian, A., et al., 2002, Nucl. Instrum. Methods Phys. Res. A 479, 117.

Abbott, B., et al., 1998, Phys. Rev. D 58, 052001.

Abbott, B., et al., 1999, Nucl. Instrum. Methods Phys. Res. A 424, 352.

Abe, F., et al., 1995, Phys. Rev. D 52, 4784.

Abe, F., et al., 1999, Phys. Rev. Lett. 82, 271.

Adinolfi, M., et al., 2002, Nucl. Instrum. Methods Phys. Res. A 482, 363.

Aharonian, F. A., et al., 2000, Astrophys. J. 539, 317.

Akchurin, N., et al., 1998, Nucl. Instrum. Methods Phys. Res. A 408, 380.

Akesson, T., et al., 1985, Nucl. Instrum. Methods Phys. Res. A 241, 17.

Akhmadaliev, S., et al., 2002, Nucl. Instrum. Methods Phys. Res. A 480, 508.

Akrawy, M., et al., 1990, Nucl. Instrum. Methods Phys. Res. A 290, 76.

Alavi-Harati, A., et al., 1999, Phys. Rev. Lett. 83, 22.

Alessandrello, A., et al., 2000, Phys. Lett. B 486, 13.

Alkhazov, G. D., et al., 1967, Nucl. Instrum. Methods 34, 1.

Altegoer, J., et al., 1998, Nucl. Instrum. Methods Phys. Res. A 404, 96.

Amaldi, U., 1981, Phys. Scr. 23, 409.

Amenomori, M., et al., 2000, Astrophys. J. 532, 302.

Andrieu, B., et al., 1993, Nucl. Instrum. Methods Phys. Res. A 336, 460.

Antonelli, A., et al., 1995, Nucl. Instrum. Methods Phys. Res. A 354, 352.

Aslanides, E., et al. (ANTARES Collaboration), 1999, A Deep Sea Telescope for High Energy Neutrinos, Internal Document DAPNIA-99-001; see also astro-phy/9907432.

ATLAS Collaboration, 1996a, Calorimeter Performance Technical Design Report (CERN/LHCC/96-40).

ATLAS Collaboration, 1996b, Liquid Argon Calorimeter Technical Design Report (CERN/LHCC/96-41).

ATLAS Collaboration, 1996c, ATLAS Tile Calorimeter Technical Design Report (CERN/LHCC/96-42).

ATLAS Collaboration, 1997, Inner Detector Technical Design Report (CERN/LHCC/97-17).

ATLAS Collaboration, 1999, Detector and Physics Performance Technical Design Report (CERN/LHCC/99-14).

Aubert, B., et al., 1990, Liquid Argon Calorimetry with LHC Performance Specifications (CERN/DRDC/90-31).

Azhgirey, I., et al., 1996, in Proceedings of the XVth Conference on Charged Particle Accelerators, Protvino, p. 74.

BaBaR Collaboration, 2000, "The First Year of the BaBar Experiment at PEP-II,” SLAC-PUB-8539.

Bakken, J. A., et al., 1985, Nucl. Instrum. Methods Phys. Res. A 228, 296.

Balka, L., et al., 1988, Nucl. Instrum. Methods Phys. Res. A 267, 272.

Balkanov, V. A., 2000, in 26th International Cosmic Ray Conference, Salt Lake City, UT, 1999, edited by B. L. Dingus, D. B. Kieda, and M. H. Salamon, AIP Conf. Proc. No. 516 (AIP, Melville, NY), Vol. 2, p. 217.

Barr, G. D., et al., 1990, Proposal for a Precision Measurement of $\epsilon^{\prime} / \epsilon$ in $C P$-Violating $K^{0} \rightarrow 2 \pi$ Decays (CERN/SPSC/90-22, SPSC/P253).

Barrio, J. A., et al., 1998, "The MAGIC Telescope: Design Study for the Construction of a $17 \mathrm{~m}$ Cherenkov Telescope 
for Gamma Astronomy above $10 \mathrm{GeV}$," version 5, MaxPlanck Inst., Munich Report MPI-PhE/98-5.

Beard, C., et al., 1990, Nucl. Instrum. Methods Phys. Res. A 286, 117.

Bebek, C., et al., 1988, Nucl. Instrum. Methods Phys. Res. A 265, 258.

Behrens, U., et al., 1990, Nucl. Instrum. Methods Phys. Res. A 289, 115.

Bertolucci, S., et al., 1988, Nucl. Instrum. Methods Phys. Res. A 267, 301.

Bhat, M. R., 1992, in Nuclear Data for Science and Technology, edited by S. M. Quaim (Springer, 1992).

Bird, D. J., et al., 1995, Astrophys. J. 441, 144.

Bond, I. H., 2000, The Very Energetic Radiation Imaging Telescope Array System (VERITAS), http:// earth.physics.purdue.edu/veritas/index.shtml

Booth, N. E., B. Cabrera, and E. Fiorini, 1996, Annu. Rev. Nucl. Part. Sci. 46, 471.

Booth, N. E., and D. J. Goldie, 1996, Supercond. Sci. Technol. 9, 493.

Boutigny, D., et al., 1995, The BaBar Technical Design Report (SLAC-R-457).

Boyer, J., et al., 2002, Nucl. Instrum. Methods Phys. Res. A 482, 457.

Buskulic, D., et al., 1995, Nucl. Instrum. Methods Phys. Res. A 360, 481.

Cabrera, B., et al., 2000, Physica B 280, 509.

CALOR conferences, 2002, http://3w.hep.caltech.edu/calor02/. Cassiday, G. L., 1985, Annu. Rev. Nucl. Part. Sci. 35, 321.

Cebrian, S., et al., 2000, Nucl. Instrum. Methods Phys. Res. A 444, 315.

Cecchini, S., 2001, Nucl. Phys. B (Proc. Suppl.) 97, 48.

Chardin, G., et al., 2000, Nucl. Instrum. Methods Phys. Res. A 444, 319.

Cleland, W. E., and E. G. Stern, 1994, Nucl. Instrum. Methods Phys. Res. A 338, 467.

Cloth, P., et al., 1988, Jülich Report JUL-2203.

CMS Collaboration, 1997, The Electromagnetic Calorimeter Project Technical Design Report (CERN/LHCC/97-33).

Crawford, H. J., et al., 1987, Nucl. Instrum. Methods Phys. Res. A 256, 47.

Cronin, J. W., K. G. Gibbs, and J. C. Weekes, 1993, Annu. Rev. Nucl. Part. Sci. 43, 883.

Décamp, A., et al., 1990, Nucl. Instrum. Methods Phys. Res. A 294, 121.

de La Taille, C., 2000, in Proceedings of the VIIIth International Conference on Calorimetry in High Energy Physics (CALOR 99), Lisbon, Portugal, edited by G. Barreira and B. Tomé (World Scientific, Singapore), p. 557.

Derrick, M., et al., 1991, Nucl. Instrum. Methods Phys. Res. A 309, 77.

Doke, T., et al., 1976, Nucl. Instrum. Methods Phys. Res. A 134, 353.

Drews, G., et al., 1990, Nucl. Instrum. Methods Phys. Res. A 290, 335.

Emmett, M. B., 1975, Oak Ridge Report ORNL-4972.

Engler, J., et al., 1999, Nucl. Instrum. Methods Phys. Res. A 427, 528.

Fabjan, C. W., 1987, in Experimental Techniques in HighEnergy Physics, edited by T. Ferbel (Addison-Wesley, Menlo Park).

Fabjan, C. W., and R. Wigmans, 1989, Rep. Prog. Phys. 52, 1519.
Fabjan, C. W., and W. J. Willis, 1975, in Proceedings of the Calorimeter Workshop, Batavia, edited by M. Atac (FNAL, Batavia, IL), p. 1.

Fano, U., 1947, Phys. Rev. 72, 26.

Fanti, V., et al., 1999, Phys. Lett. B 465, 335.

Fasso, A., et al., 1983, Nucl. Instrum. Methods Phys. Res. A 332, 459.

Ferrari, A., 2001, private communication.

Ferrari, A., and P. R. Sala, 2001, in Proceedings of the 9th International Conference on Calorimetry in High Energy Physics, Annecy, France, 2000, Frascati Physics Series 21, edited by B. Aubert et al. (INFN, Frascati), p. 31.

Fesefeldt, H. C., 1985, University of Aachen Technical Report PITHA 85-02.

Fiorini, E., 2000, Nucl. Instrum. Methods Phys. Res. A 444, 65. Fischer, H. G., 1978, Nucl. Instrum. Methods 156, 81.

Gabriel, T. A., and J. D. Amburger, 1974, Nucl. Instrum. Methods 116, 33.

Gabriel, T. A., et al., 1994, Nucl. Instrum. Methods Phys. Res. A 338, 336.

GEANT4 Collaboration, 1994, CERN/DRDC/94-29.

Greisen, K., 1966, Phys. Rev. Lett. 16, 748.

Griedler, P. F. K., Nestor Collaboration, 2001, Nucl. Phys. B (Proc. Suppl.) 97, 105.

Groom, D. E., 1998, in Proceedings of the 7th International Conference on Calorimetry in High Energy Physics, Tucson, AZ, edited by E. Chen et al. (World Scientific, Singapore), p. 507.

Haller, E., 1995, J. Appl. Phys. 77, 2857.

Hallgren, A. (AMANDA Collaboration), 2002, Nucl. Phys. B, Proc. Suppl. 110, 507.

Hayashida, N., et al., 1994, Phys. Rev. Lett. 73, 3491.

Heck, D., et al., 1999, FZKA report 6019.

Hellmig, J., et al., 2000, Nucl. Instrum. Methods Phys. Res. A 444, 308 .

Hill, G., 2000, in Proceedings of the 26th International Cosmic Ray Conference, Salt Lake City, UT, 1999, edited by B. L. Dingus, D. B. Kieda, and M. H. Salamon (AIP, Melville, NY), H.E. 6.3.04.

Hill, G. C., et al. (AMANDA Collaboration), 2001, preprint astro-ph/0106064, and Proceedings of the 36th Recontres de Moriond, Moriond.

Hillas, A. M., et al., 1971, in Proceedings of the 12th International Cosmic Ray Conference, Hobart, Vol. 3, p. 1001.

Hoffman, C. M., C. Sinnis, and P. Fleury, 1999, Rev. Mod. Phys. 71, 897.

Hughes, E. B., 1972, IEEE Trans. Nucl. Sci. 19, 126.

Irwin, K. D., et al., 1995, Appl. Phys. Lett. 66, 1998.

Kampert, K.-H., et al., 2001, in Proceedings of the 27th International Cosmic Ray Conference, Hamburg (Copernic Gesellschaft, Katlenburg-Lindau), preprint astro-ph/0204205.

Karyotakis, Y., 1995, "The L3 electromagnetic calorimeter," LAPP-EXP-95-02, paper contributed to the Proceedings of the 1994 Beijing Calorimetry Symposium, Beijing, China, edited by Chen He Sheng (Beijing Univ. Inst. High-Energy Physics, Beijing).

Kelley, R. L., et al., 2000, Nucl. Instrum. Methods Phys. Res. A 444, 170.

Kim, Y.-K., 1999, in Proceedings of the XIIIth Rencontres de Physique de la Vallée d'Aoste, Results and Perspectives in Particle Physics, La Thuile, Italy, edited by M. Greco (INFN, Frascati), Frascati Physics Series, Vol. 14. 
Kling, A., F. Bãrao, M. Nakagawa, L. Távora, and P. Vaz, 2001, Eds., Advanced Monte Carlo for Radiation Physics, Particle Transport Simulation and Applications, in Proceedings of the Monte Carlo 2000 Conference, Lisbon (Springer, Berlin).

Knapp, D., D. Heck, S. J. Sciutto, M. T. Dova, and M. Risse, 2003, Astropart. Phys. 19, 77.

Knoll, G. F., 1989, Radiation Detection and Measurements, 2nd ed. (Wiley, New York).

Konopelko, A., et al., 1999, Astropart. Phys. 10, 275.

Kulchitsky, Y. A., 2000, in Proceedings of the VIIIth International Conference on Calorimetry in High Energy Physics, Lisbon, 1999, edited by G. Barreira and B. Tomé (World Scientific, Singapore), p. 401.

Longo, E., and I. Sestili, 1975, Nucl. Instrum. Methods 128, 283.

Maire, M., 2001, in Proceedings of the 9th International Conference on Calorimetry in High Energy Physics (CALOR 2000), Annecy, France, edited by B. Aubert et al. (INFN, Frascati) Frascati Physics Series 21.

Martinez, M. (MAGIC Collaboration), 1998, The MAGIC Telescope, in Proceedings of the 16th European Cosmic Ray Symposium, Alcala de Henares, Madrid, pp. 397-400.

Martini, M., 1998, in Proceedings of the VIIth International Conference on Calorimetry in High-Energy Physics (CALOR 97), Tucson, edited by E. Cheu et al. (World Scientific, Singapore).

McCammon, D., W. Cui, M. Juda, J. Morganthaler, J. Zhang, B. L. Kelley, S. S. Holt, G. M. Madejski, S. H. Mosely, and A. E. Szymkowiak, 1993, Nucl. Instrum. Methods Phys. Res. A 326, 157.

Mohanty, G., et al., 1998, Astropart. Phys. 9, 15.

Murzin, V. S., 1967, Prog. Elem. Part. Cosmic Ray Phys. 9, 247. NA48 Collaboration, 1995, Nucl. Instrum. Methods Phys. Res. A 360, 224.

Nakagawa, T., et al., 1995, J. Nucl. Sci. Technol. 32, 1259.

Nelson, W. R., H. Hirayama, and D. W. O. Rogers, 1985, Report SLAC-265.

Ootani, W., et al., 1999, Nucl. Instrum. Methods Phys. Res. A 436, 233.

Particle Data Group, 2002, Phys. Rev. D 66, 010001.

Peacock, A., 1999, Physica B 263, 595.

Pi, H., 1992, FRITIOF, version 7.0, Comput. Phys. Commun. 71, 173.

Porter, F. S., et al., 2002, Eds., Low-Temperature Detectors, AIP Conf. Proc. No. 605 (AIP, Melville, NY).

Pretzl, K., 2000, Nucl. Instrum. Methods Phys. Res. A 454, 114.
Pryke, C. L., 1998, Auger Project Technical Note GAP-98-006. Rando, N., et al., 2000, Nucl. Instrum. Methods Phys. Res. A 444, 441.

Ranft, J., 1970, Nucl. Instrum. Methods 81, 29.

Ranft, J., 1997, DAMJET (INFN/AE-97/45).

Ranft, J., 2001, in Proceedings of the Monte Carlo 2000 Conference, Lisbon, edited by A. Kling, F. Bãrao, M. Nakagawa, L. Távora, and P. Vaz (Springer, Berlin), p. 1021.

Rossi, B., 1952, High Energy Particles (Prentice Hall, Englewood Cliffs, NJ).

Schacht, P., 2000, in Proceedings of the VIIIth International Conference on Calorimetry in High Energy Physics (CALOR 99), Lisbon, Portugal, edited by G. Barreira and B. Tomé (World Scientific, Singapore).

Seguinot, J., J. Tischhauser, and T. Ypsilantis, 1995, Nucl. Instrum. Methods Phys. Res. A 354, 280.

Seman, M., 2001, private communication.

Sisti, M., et al., 2000, Nucl. Instrum. Methods Phys. Res. A 444, 312.

Spiering, C., 1999, Ed., Proceedings of Simulation and Analytic Methods for Large Neutrino Telescopes (DESY-PROC-199901).

Super-Kamiokande Collaboration, 1999, Nucl. Instrum. Methods Phys. Res. A 421, 113.

Takeda, M., et al., 1998, Phys. Rev. Lett. 81, 1163.

Takeda, M., et al., 1999, Astrophys. J. 522, 225.

Tsai, Y. S., 1974, Rev. Mod. Phys. 46, 815.

Twerenbold, D., 1996, Rep. Prog. Phys. 59, 349.

Unal, G., 2001, in Proceedings of the 9th International Conference on Calorimetry in High Energy Physics (CALOR 2000), Annecy, France, edited by B. Aubert et al. (INFN, Frascati), Frascati Physics Series 21, p. 361.

van den Brandt, B., et al., 2000, Nucl. Phys. B (Proc. Suppl.) 87, 117.

Wellisch, J. P., 1999, Nucl. Phys. B 78, 738.

Wienke, H., and M. Herman, 1998, International Atomic Energy Agency Report IAEA-NDS-176.

Wigmans, R., 1988, Nucl. Instrum. Methods Phys. Res. A 265, 273.

Wigmans, R., 2000, Calorimetry: Energy Measurement in Particle Physics (Clarendon Press, Oxford).

Yakutsk Extensive Air Shower Array, 2001, http:// www.intas.be/beyond/icra0001.htm

Zatsepin, G. T., and V. A. Kuzmin, 1966, JETP Lett. 4, 78.

Zeitnitz, C., and A. Gabriel, 1996, The GEANT-Calor Interface User's Guide (ORNL, Oak Ridge). 